\title{
Localization Principle for Differential Complexes and Its Application
}

\author{
By \\ Isao NARUKI
}

\section{Introduction}

This paper gives a full account of the theory which was outlined in the author's previous paper [12].

It is usual to solve the so-called $D$-Neumann problem in sufficiently small balls when one wants to prove the exactness in sheaf level, of the Spencer sequence for a differential equation $D u=0$ with unknown $u$ (Sweeney [16]). In this direction an essential development has been made recently for some reasonable class of elliptic systems (MacKichan $[10]$, Sweeney [17]). However in the non-elliptic case very little was known yet concerning the Neumann problem, and there are many important differential equations which are not elliptic; for example, the tangential Cauchy-Riemann equation associated with a real submanifold in a complex manifold, is certainly not elliptic. In the author's knowledge, it seems no one has succeeded in solving the Neumann problem (in sufficiently small balls) for such an equation. Is there any other approach to prove the exactness in this case? Fortunately the tangential CauchyRiemann equation has some good nature because of the intimate relation to the complex analysis in several variables, so that many techniques in the complex analysis should be applied to the study of such an equation.

With this fact in mind, we attempt here to present a new method for proving exactness or partial exactness of a complex of first order differential operators between vector bundles, provided that this complex admits sufficiently many functions which, when regarded as scalar multiplication operators, commute with the differentiation of the complex. (Such Received October 7, 1971. 
a function is called analytic with respect to the complex.) This is the localization principle which is based on the idea of the Oka map, and we mainly concern ourselves with this in $\S 1$. The word "localization" comes from the procedure to apply this principle (\$1.2-1.3): We first fix a point of the manifold on which the complex is defined, and cut out a small neighbourhood of this point and situate it into some good free space, extending the complex outside this neighbourhood so that suitable partial homotopies can be easily constructed for the resulting complex on this free space. Next we localize these homotopies to a smaller neighbourhood like an analytic polyhedron, in which the partial exactness of the complex is thus proved. If the analytic functions are so many that one can make such a neighbourhood arbitrarily small, then the partial exactness follows also in sheaf level at that point. Here we have meant by a "partial homotopy", a partial homotopy for complexes such as constituted by spaces of sections satisfying some growth condition at the infinity. These conditions, however, are rather technical and disappear finally by the localization. To introduce partial homotopies we employed here the functional analytic method of Hörmander [4], [5] relying on a priori estimates concerning some Dirichlet norms. But this gives only auxiliary partial homotopies, and the essential step consists in the choice of the appropriate growth conditions to give actually suitable partial homotopies to which our principle can be applied.

In $\S 2$, we shall apply the result of $\S 1$ to the Dolbeault type sequences for real submanifolds, and prove some partial exactness of these sequences, as well as the dual version of this partial exactness. These sequences were introduced by Kohn-Rossi [9] for hypersurfaces, but the generalization to the case of higher codimension is obvious (\$2.1). They coincide either with the Spencer sequence for the tangential Cauchy-Riemann equation, or with the product of a finite number of its copies. The standard real submanifolds in the sense of Tanaka [18] are basic in the study of general real submanifolds, in fact when perturbed suitably on a compact subset, they serve as the good free spaces indicated above. The process to cut out neighbourhoods to be situated in these free spaces, can be done by a local approximation of real submanifolds by standard ones (Lemma 
2.5.2). The a priori estimate for deriving partial homotopies in each of these free spaces, can be obtained from a simple estimate for the Dolbeault type sequences associated with the standard real submanifold, and from a general subellipticity theorem concerning the Dolbeault sequences. The a priori estimate for the standard one involves the Dirichlet norm defined by the partial Gaussian measure depending on a real parameter. In proving this estimate the most crucial point is to remedy the difficulty arising from the involvement between the Gaussian measure and the Levi form. The subellipticity theorem mentioned above, was proved by Kohn [8] in case the real codimension equals 1 ; in case of higher codimension this was proved by Hörmander [6] in a general frame work on subellipticity. This subellipticity theorem plays the role to ensure the stability of the estimates for standard real submanifolds under a small perturbation on a compact subset, so that these estimates can be modified to fit for the free spaces indicated above.

At the end of this paper we give two appendices. Appendix 1 consists only of supplements to $\S 2$. In Appendix 2 we give a simple application of our localization principle to the Spencer sequences of some elliptic differential equations with constant coefficients. This seems to suggest that the applicability of our principle is not restricted to the tangential Cauchy-Riemann equations.

Throughout this paper we assume the differentiability of class $C^{\infty}$ for manifolds, vector bundles, differential operators and so on, unless the contrary is stated explicitly. When $F$ is a vector bundle over a manifold $M, C^{\infty}(F)$ denotes the set of smooth sections of $F$ over $M$, whereas $C^{\infty}(U, F)$ denotes the set of smooth sections over $U$ if $U$ is an open subset of $M$. We denote by $\Gamma(S, G)$ the set of sections over $S$ of a sheaf $G$ over a manifold of which $S$ is a subset.

The author should like to express his sincere gratitude to Professors S. Matsuura, M. Sato and N. Tanaka for their critical advices and constant encouragement. He also thanks his colleagues T. Kawai and M. Kashiwara for their stimulating conversations. 


\section{Localization Principle}

§1.1. Basic notions and formulas. We shall first give and fix a complex

$$
\cdots \longrightarrow E^{i-1} \stackrel{\partial^{i-1}}{\longrightarrow} E^{i} \stackrel{\partial^{i}}{\longrightarrow} E^{i+1} \longrightarrow \cdots
$$

where $E^{i}, i \in \mathbb{Z}$ are vector bundles over a manifold $M$ and $\partial^{i}: E^{i} \rightarrow E^{i+1}$ are first order differential operators such that $\partial^{i+1} \cdot \partial^{i}=0$. For brevity we denote this complex by $E^{\cdot}$ and also denote by $C^{\infty}\left(E^{*}\right)$ the corresponding complex $\cdots \rightarrow C^{\infty}\left(E^{i-1}\right) \rightarrow C^{\infty}\left(E^{i}\right) \rightarrow C^{\infty}\left(E^{i+1}\right) \rightarrow \cdots$. If $\cdots \rightarrow A^{i-1} \rightarrow A^{i} \rightarrow A^{i+1}$ $\rightarrow \cdots$ is a subcomplex of $C^{\infty}\left(E^{\cdot}\right)$, then we simply write $A^{\cdot}$ for this complex.

Definition 1.1.1. A subcomplex $A^{\cdot}$ of $C^{\infty}\left(E^{\cdot}\right)$ is called topological if and only if (1) $A^{i}, i \in \mathbb{Z}$ are locally convex topological vector spaces such that the inclusions $A^{i} \hookrightarrow C^{\infty}\left(E^{i}\right)$ are continuous and (2) $\partial^{i} \mid A^{i}$ : $A^{i} \rightarrow A^{i+1}$ are continuous for these topologies. When each $A^{i}$ is complete, we say that $A^{\cdot}$ is complete.

Throughout this paper we require all subcomplexes of $C^{\infty}\left(E^{\cdot}\right)$ to be topological without mentioning this word explicitly. This assumption is not so restrictive since every algebraic subcomplex can be made a topological one by inducing the topology from $C^{\infty}\left(E^{\cdot}\right)$.

Definition 1.1.2. Let $A^{*}, B^{\cdot}$ be two subcomplexes of $C^{\infty}\left(E^{\cdot}\right)$ such that $A^{\cdot} \subset B^{\cdot}$ and let $s^{i}, i \leqq q$ be continuous linear maps from $A^{i}$ to $B^{i-1}$. The family of maps $s=\left(s^{i}\right)_{i \leqq q}$ is said to be a $(-\infty, q)$-homotopy for the inclusion $A^{\cdot} \hookrightarrow_{\hookrightarrow} B \cdot$ if and only if (1) $\left(\partial^{i-1} s^{i}+s^{i+1} \partial^{i}\right) u=u$ for $i<q$ and for $u \in A^{i}$, and (2) $\partial^{q-1} s^{q} u=u$ for $u \in A^{q}$ such that $\partial^{q} u=0$.

Note here that the inclusions $A^{i} \hookrightarrow B^{i}, i<q$ are continuous if there is a $(-\infty, q)$-homotopy for $A^{\cdot} \hookrightarrow B^{\circ}$.

Definition 1.1.3. Let $A^{\bullet}, B^{\bullet}, C^{\cdot}$ be subcomplexes of $C^{\infty}\left(E^{\cdot}\right)$ such that $A^{\cdot} \subset B^{\circ} \subset C^{\cdot}$. Let further $s^{i}, i>q$ be continuous linear maps from 
$C^{i}$ into $C^{i-1}$ and $s^{q}$ a continuous linear map from $C^{q}$ into $C^{\infty}\left(E^{q-1}\right)$. The family of maps $s=\left(s^{i}\right)_{i \geqq q}$ is called a $(q, \infty)$-homotopy of $C \cdot$ modulo $\left(A^{\cdot}, B^{\cdot}\right)$ if and only if (1) $s^{i}\left(A^{i}\right) \subset B^{i-1}$ for $i>q$ and (2) $\left(\partial^{i-1} s^{i}+s^{i+1}\right.$ $\left.\partial^{i}\right) u \equiv u$ modulo $B^{i}$ for $i \geqq q$ and for $u \in C^{i}$.

In this definition the validity of being $(q, \infty)$-homotopy of $s$ is independent of the topologies of $A^{*}$ and $B^{*}$, so we shall apply this definition even to the case $A^{*}, B^{\cdot}$ are merely algebraic subcomplexes. Note also that the conditions in Definitions 1.1 .2 and 1.1 .3 are a little delicate at the extreme point $i=q$; the naturality of these will be understood from the argument in the next section.

Now let $F$ be a vector bundle over $M$ and $\Omega$ be an open subset in the complex plane $\mathbf{C}$. Then we have the canonical identification $C^{\infty}(\Omega$, $\left.C^{\infty}(F)\right)=C^{\infty}(\Omega \times M, \tilde{F})$ where $\tilde{F}$ is the pull back of the bundle $F$ by the projection $\pi: \mathbf{C} \times M \rightarrow M$. Given a smooth function $f$ on $M$, we obtain a map which assigns an element, denoted by $[V]_{f}$, of $C^{\infty}(F)$ to each $V \in C^{\infty}\left(\mathbf{C}, C^{\infty}(F)\right)$ by setting

$$
[V]_{f}=\tilde{\pi} \cdot V \cdot\left(f \times 1_{M}\right)
$$

where $1_{M}$ is the identity map of $M$ and $\tilde{\pi}$ is the projection from $\tilde{F}$ onto $F$. For a locally convex space $A, C^{\infty}(\Omega, A)$ denotes the space of smooth $A$-valued functions on $\Omega$ with the topology of uniform convergence on compact subsets for the semi-norms defining the topology of $A$. If $A \hookrightarrow$ $C^{\infty}(F)$ is continuous, then $C^{\infty}(\Omega, A)$ is a subsapce of $C^{\infty}(\Omega \times M, \tilde{F})=C^{\infty}(\Omega$, $\left.C^{\infty}(F)\right)$ and the inclusion $C^{\infty}(\Omega, A) \hookrightarrow C^{\infty}\left(\Omega, C^{\infty}(F)\right)$ is continuous.

Definition 1.1.4. Let $A^{\cdot}$ be a subcomplex of $C^{\infty}\left(E^{\cdot}\right)$. A function $f \in C^{\infty}(M)$ is $A^{\circ}$-admissible if and only if the following conditions are fulfilled for each $i \in \mathbf{Z}$ :

(1) $f u, \bar{f} u, \varphi(f) u \in A^{i}$ for any $u \in A^{i}$ and for $\varphi \in C_{0}^{\infty}(\mathbf{C})$.

(2) $\chi(\zeta) \varphi(f) u /(\zeta-f) \in C_{0}^{\infty}\left(\mathbf{C}, A^{i}\right)$ for $u \in A^{i}$ and for $\varphi, \chi \in C_{0}^{\infty}(\mathbf{C})$ such that supp $\varphi \cap \operatorname{supp} x=\phi$.

(3) $[V]_{f} \in A_{i}$ for every $V \in C_{0}^{\infty}\left(\mathbf{C}, A^{i}\right)$.

(4) The above maps $u \mapsto f u, u \mapsto \bar{f} u, u \mapsto \varphi(f) u \quad u \mapsto \chi(\zeta) \varphi(f) u /$ $(\zeta-f), V \mapsto[V]_{f}$ are continuous. 
Definition 1.1.5. A function $f \in C^{\infty}(V)$ on an open subset $U$ of $M$ is called $E^{\cdot}$-analytic in $U$ if and only if $\partial^{i}(f u)=f \partial^{i} u$ for any $i \in Z$ and $u \in C^{\infty}\left(U, E^{i}\right)$.

The $E^{\cdot}$-analyticity is a local property and the product of two $E^{\cdot}$-analytic functions is again $E^{\cdot}$-analytic, in fact, there is a way to define $E^{*}$-analytic functions to be solutions of a homogeneous first order differential equation: Let $\sigma\left(\partial^{i}\right)$ denote the principal symbol of the differential operator $\partial^{i}$. We interpret here $\sigma\left(\partial^{i}\right)$ as an operater of order 0 of $T^{*}$ into Hom $\left(E^{i}, E^{i+1}\right)$. ( $T^{*}$ denotes the complexified cotangent bundle of M.) Define for $f \in C^{\infty}(U)$ an operator $\vartheta^{i}(f)$ of order 0 from $E^{i} \mid U$ to $E^{i+1} \mid U$ by setting $\vartheta^{i}(f)=\sigma\left(\partial^{i}\right) \cdot d f$. Then

$$
\partial^{i}(f u)-f \partial^{i} u=\vartheta^{i}(f) u \quad u \in C^{\infty}\left(U, E^{i}\right) .
$$

The operator $\vartheta^{i}$ is a first order differential operator from the trivial bundle of rank 1 to $\operatorname{Hom}\left(E^{i}, E^{i+1}\right)$, and $f$ is $E^{\cdot}$-analytic in $U$ if and only if

$$
\vartheta^{i}(f)=0 \quad i \in Z .
$$

We denote by $A\left(U, E^{\cdot}\right)$ the ring of $E^{\cdot}$-analytic functions in $U$ and write $A\left(E^{*}\right)$ instead of $A\left(M, E^{\cdot}\right)$. The ring $A\left(U, E^{*}\right)$ is an analytic ring in the sense of M. Sato, that is, for every entire analytic function $\psi\left(z_{1}, \ldots\right.$, $\left.z_{n}\right)$ and for $\left(f_{1}, \ldots, f_{n}\right) \in A\left(U, E^{\cdot}\right)^{n}$, the function $\psi\left(f_{1}, \ldots, f_{n}\right)$ is also $E^{\cdot}$-analytic in $U$. In particular $1 /(\zeta-f)\left(\zeta \in \mathbb{C}, f \in A\left(E^{\cdot}\right)\right)$ is $E^{\cdot}$-analytic where $f \neq \zeta$.

Finally we conclude this section by mentioning several formulae which are used frequently in the next section: From (1.1.1) it follows immediately

$$
\partial^{i+1} \vartheta^{i}(f) u=-\vartheta^{i+1}(f) \partial^{i} u \quad u \in C^{\infty}\left(E^{i}\right) .
$$

By using this and noticing that $\vartheta^{i}(f), i \in Z$ are $C^{\infty}(M)$-linear,

$$
\begin{aligned}
\vartheta^{i+1}(f) \vartheta^{i}(f) u & =\partial^{i+1}\left(f \vartheta^{i}(f) u\right)-f \partial^{i+1} \vartheta^{i}(f) u \\
& =\partial^{i+1} \vartheta^{i}(f) f u+f \vartheta^{i+1}(f) \partial^{i} u \\
& =-\vartheta^{i+1}(f) \partial^{i}(f u)+\vartheta^{i+1}(f) f \partial^{i} u
\end{aligned}
$$




$$
\begin{aligned}
& =-\vartheta^{i+1}(f)\left(\partial^{i}(f u)-f \partial^{i} u\right) \\
& =-\vartheta^{i+1}(f) \vartheta^{i}(f) u
\end{aligned}
$$

whence it follows that

$$
\vartheta^{i+1}(f) \cdot \vartheta^{i}(f) u=0 \quad u \in C^{\infty}\left(E^{i}\right)
$$

For every $V(\zeta), \zeta \in \mathbf{C}$ of $C^{\infty}\left(\mathbf{C}, C^{\infty}\left(E^{i}\right)\right)$ we have

$$
\partial^{i}[V]_{f}=\left[\partial^{i} V\right]_{f}+\vartheta^{i}(f)[\partial V / \partial \zeta]_{f}+\vartheta^{i}(\bar{f})[\partial V / \partial \bar{\zeta}]_{f}
$$

(This can be checked directly for elements of $C^{\infty}\left(\mathbf{C}, C^{\infty}\left(E^{i}\right)\right)$ of the form $\varphi u$ where $\varphi \in C^{\infty}(\mathbf{C}), u \in C^{\infty}\left(E^{i}\right)$; then the general case follows immediately by approximating a given $V \in C^{\infty}\left(\mathbf{C}, C^{\infty}\left(E^{i}\right)\right)$ in the $C^{\infty}$-topology by linear combinations of such elements.) From (1.1.3) and (1.1.4) we obtain the following

Lemma 1.1.6. If $f$ is $E^{\cdot}$-analytic, then

$$
\partial^{i+1} \vartheta^{i}(\bar{f})[V]_{f}=-\vartheta^{i+1}(\bar{f})\left[\partial^{i} V\right]_{f} \quad V \in C^{\infty}\left(\mathbf{C}, C^{\infty}\left(E^{i}\right)\right)
$$

$\S 1.2$. Fundamental theorems. To state the fundamental theorems we still need some notation: Let $K$ be a closed subset of $M$, and $A^{*}$ a subcomplex $C^{\infty}\left(E^{\cdot}\right)$. Then $A \cdot\{K\}$ is a subcomplex of $A^{\cdot}$ whose terms $A^{i}\{K\}, i \in Z$ are given by

$$
A^{i}\{K\}=\left\{u \in A^{i} \mid \operatorname{supp} u \subset K\right\}
$$

If $A^{\cdot}$ is complete, then $A^{\cdot}\{K\}$ is also complete. Let now $f=\left(f_{1}, f_{2}, \ldots\right.$, $f_{\rho}$ ) be a $\rho$-tuple of functions on $M$. Then $P(f)$ denotes the open set $\left\{z \in M|| f_{1}(z)|<1, \ldots,| f_{\rho}(z) \mid<1\right\}$ and $P[f]$ denotes the closed set $\left\{z \in M|| f_{1}(z)|\leqq 1, \ldots,| f_{\rho}(z) \mid \leqq 1\right\}$. Now our main theorems are stated as follows.

Theorem 1.2.1. Let $f=\left(f_{1}, \ldots, f_{\rho}\right)$ be a $\rho$-tuple of $E^{\cdot}$-analytic functions which are all $A^{\cdot}$-admissible for a complete subcomplex $A^{\cdot}$ of $C^{\infty}\left(E^{*}\right)$. Suppose that $s=\left(s_{i}\right)_{i \leqq q}$ is a continuous $(-\infty, q)$-homotopy for the identity 
inclusion $A^{\circ} \hookrightarrow A^{\circ}$. Then one can construct for any $a>1, a(-\infty, q)$ homotopy $\sigma=\left(\sigma^{i}\right)_{i \leqq q}$ for the inclusion $A \cdot\{P[a f]\} \hookrightarrow_{\rightarrow} A \cdot\{P[f]\}$.

Theorem 1.2.2. Let $f=\left(f_{1}, \ldots, f_{\rho}\right)$ be a $\rho$-tuple of $E^{\cdot}$-analytic functions which are all $C^{\cdot}$-admissible for a subcomplex $C^{\cdot}$ of $C^{\infty}\left(E^{\cdot}\right)$ and suppose that $s=\left(s^{i}\right)_{i \leqq q}$ is a $(q, \infty)$-homotopy of $C \cdot$ modulo $(0,0)$. Then there is for $a>1$ a $(q, \infty)$-homotopy $\sigma=\left(\sigma^{i}\right)_{i \geqq q}$ of $C \cdot$ modulo $\left(C \cdot\left\{P(f)^{c}\right\}\right.$, $\left.C \cdot\left\{P(a f)^{c}\right\}\right)$ where $P(f)^{c}$ and $P(a f)^{c}$ denote the complements in $M$ of $P(f), P(a f)$ respectively.

The proofs of these theorems proceed by the induction on $\rho$; the passage from the case $\rho=k$ to the case $\rho=k+1$ will be done for each $k=0,1,2, \cdots$ by the following lemmas.

Lemma 1.2.3. Let $A_{\dot{1}}, A_{\dot{2}}, A_{\dot{3}}$ be three complete subcomplexes of $C^{\infty}\left(E^{\cdot}\right)$ with inclusions $A_{1} \hookrightarrow A_{2}, A_{2} \hookrightarrow A_{3} . \quad$ Suppose that $s_{1}=\left(s_{1}^{i}\right)_{i \leqq q}$ and $s_{2}=\left(s_{2}^{i}\right)_{i \leqq q}$ are $(-\infty, q)$-homotopies for the inclusion $A_{1} \hookrightarrow A_{2}$ and for the inclusion $A_{2} \hookrightarrow A_{3}^{*}$ respectively. If an $E^{\cdot}$-analytic function $f$ is $A_{i}^{*}$-admissible for $i=1,2,3$, then there is for each $a>1, a(-\infty, q)$-homotopy $s=\left(s^{i}\right)_{i \leqq q}$ for the inclusion $A_{i}\{P[a f]\} \hookrightarrow A_{3}\{P[f]\}$.

Lemma 1.2.4. Let $B_{1}, B_{2}^{*}, B_{3}^{*}$ be closed subcomplexes of a subcomplex $C^{\cdot}$ of $C^{\infty}\left(E^{\cdot}\right)$ with inclusions $B_{1} \subset B_{2} \subset B_{3}^{\cdot}$, and assume that $s_{\alpha}=$ $\left(s_{\alpha}^{i}\right)_{i \geqq q}, \alpha=1,2$ are $(q, \infty)$-homotopies of $C \cdot$ modulo $\left(B_{\alpha}^{\cdot}, B_{\alpha+1}^{\cdot}\right)$. If an $E^{\cdot}$-analytic function $f$ is $C^{\cdot}$-admissible as well as $B_{\alpha}^{\cdot}$-admissible for $\alpha=$ $1,2,3$, then there is for each $a>1, a(q, \infty)$-homotopy $s=\left(s^{i}\right)_{i \geqq q}$ modulo $\left(B_{\mathrm{i}}+C \cdot\left\{P(f)^{c}\right\}, B_{3}+C \cdot\left\{P(a f)^{c}\right\}\right)$.

Proof of Lemma 1.2.3. For a positive number $c$ we denote by $D_{c}$ the disc $|\zeta|<c$ in the complex plane $C$. Set $b=1 / a(<1)$ and choose $\psi, \varphi, x \in C_{0}^{\infty}(\mathbf{C})$ so that $\psi=1$ in a neighbourhood of $\bar{D}_{a}, \varphi=1$ in a neighbourhood of $\operatorname{supp} \psi, \chi=1$ in a neighbourhood of $\operatorname{supp} \varphi, \operatorname{supp} \chi \subset D_{1}$. Now assume that $u \in A_{1}^{i}\{P[a f]\}, i \leqq q$. Then the $A_{i}$-admissibility of $f$ implies

$$
(\partial \varphi / \partial \zeta) u /(\zeta-f)=(\partial \varphi / \partial \zeta) \psi(f) u /(\zeta-f) \in C_{0}^{\infty}\left(\mathbf{C}, A_{1}^{i}\right)
$$


In view of this we first define $J_{0}^{u}(\zeta) \in C_{0}^{\infty}\left(D_{1}, A_{2}^{i-1}\right)$ by setting

$$
J_{0}^{u}(\zeta)=(\zeta-f) s_{1}^{i}((\partial \varphi(\zeta) / \partial \zeta) u(\zeta-f)) \quad \zeta \in \mathbf{C} .
$$

Note that this is actually well defined by $A_{2}$-admissibility of $f$. We further set

$$
\begin{aligned}
& J_{1}^{u(\zeta)}=\frac{\chi(\zeta)}{2 \pi i} \int \frac{J_{0}^{u}(\zeta)}{\xi-\zeta} d \xi d \xi \\
& J_{2}^{u}(\zeta)=\frac{\partial \chi(\zeta) / \partial \zeta}{2 \pi i} \int \frac{J_{0}^{u}(\xi)}{\xi-\zeta} d \xi d \xi
\end{aligned}
$$

to define $J_{1}^{u}, J_{2}^{u} \in C_{0}^{\infty}\left(D_{1}, A_{2}^{i-1}\right)$. Here the domain of integration has been taken as the whole plane $\mathrm{C} ; J_{1}^{u}, J_{2}^{u}$ certainly belong to $C_{0}^{\infty}\left(D_{1}, A_{2}^{i-1}\right)$ since, $A_{2}^{i-1}$ being complete, the integrals always exist in $A_{2}^{i-1}$ for each $\zeta \in \mathbf{C}$. (This is the only reason why we should assume the completeness of subcomplex $A^{\cdot}$ in Theorem 1.2.1.) Now we set

$$
s^{i} u=\left[J_{1}^{u}\right]_{f}+\vartheta^{j-2}(\bar{f})\left[s_{2}^{i-1} J_{2}^{u}\right]_{f}
$$

as a definition of the map $s^{i}: A_{1}^{i}\{P[a f]\} \rightarrow A_{3}^{i-1}\{P[f]\}, i \leqq q$. Since $s_{1}$ is a $(-\infty, q)$-homotopy, the inclusion $A_{2}^{i-1} \subset A_{3}^{i-1}$ is continuous as remarked after Definition 1.1.2, which actually ensures that the first term in (1.2.1) belongs to $A_{3}^{i-1}$; the second term also belongs to $A_{3}^{i-1}$ by combining formula (1.1.1) (with $i, f$ replaced by $i-2, \bar{f}$ ) with the $A_{3}$-admissibility of $f$. That $s^{i} u \in A_{3}^{i-1}\{P(f)\}$ follows immediately from the fact that $J_{1}^{u}, J_{2}^{u} \in C_{0}^{\infty}\left(D_{1}, A_{2}^{i-1}\right)$.

Now we may only check $\left(\partial^{i-1} s^{i}+s^{i+1} \partial^{i}\right) u=u$ when $u \in A^{i}\{P[a f]\}$, $i<q$ and $\partial^{q-1} s^{q} u=u$ when $u \in A_{1}^{q}\{P[a f]\}$ satisfies $\partial^{q} u=0$. First suppose that $u \in A^{i}\{P[a f]\}, i<q$. Note that

$$
\frac{\partial}{\partial \bar{\xi}}\left(\frac{1}{2 \pi i} \int \frac{\psi(\xi)}{\xi-\zeta} d \xi d \xi\right)=\psi(\zeta)
$$

if $\phi \in C_{0}^{\infty}(\mathbf{C})$, hence

$$
\begin{aligned}
\partial J_{1}^{u}(\zeta) / \zeta & =J_{2}^{u}(\zeta)+\chi(\zeta) J_{0}^{u}(\zeta) \\
& =J_{2}^{u}(\zeta)+\chi(\zeta)(\zeta-f) s_{1}^{i}((\partial \varphi / \partial \zeta) u /(\zeta-f))
\end{aligned}
$$


Thus we obtain by noting $[(\zeta-f) *]_{f}=0$,

$$
\left[\partial J_{1}^{u}(\zeta) / \partial \xi\right]_{f}=\left[J_{2}^{u}(\zeta)\right]_{f}
$$

Using (1.1.4) and Lemma 1.1.6 we obtain from this

$$
\begin{aligned}
\partial^{i-1} s^{i} u & =\left[\partial^{i-1} J_{1}^{u}\right]_{f}+\vartheta^{i-1}(\bar{f})\left[\partial J_{1}^{u}(\zeta) / \partial \xi-\partial^{i-2} s_{2}^{i-1} J_{2}^{u}\right]_{f} \\
& =\left[\partial^{i-1} J_{1}^{u}\right]_{f}+\vartheta^{i-1}(\bar{f})\left[J_{2}^{u}-\partial^{i-2} s_{2}^{i-1} J_{2}^{u}\right]_{f} \\
& =\left[\partial^{i-1} J_{1}^{u}\right]_{f}+\vartheta^{i-1}(f)\left[s_{2}^{i} \partial^{i-1} J_{2}^{u}\right]_{f} .
\end{aligned}
$$

Hence

$$
\begin{aligned}
\left(\partial^{i-1} s^{i}+s^{i+1} \partial^{i}\right) u=\left[\partial^{i-1} J_{1}^{u}+J_{1}^{\partial^{i} u}\right]_{f} \\
\\
+\vartheta^{i-1}(\bar{f})\left[s_{2}^{i}\left(\partial^{i-1} J_{2}^{u}+J_{2}^{\partial^{i} u}\right)\right]_{f}
\end{aligned}
$$

To compute the terms in the right hand side, we first observe

$$
\begin{aligned}
\partial^{i-1} J_{0}^{u} & +J_{0}^{\partial^{i} u} \\
& =(\zeta-f)\left(\partial^{i-1} s_{1}^{i}+s_{1}^{i+1} \partial^{i}\right)(\partial \varphi(\zeta) / \partial \zeta u /(\zeta-f)) \\
& =\partial \varphi(\zeta) / \partial \zeta u
\end{aligned}
$$

whence

$$
\begin{aligned}
\partial^{i-1} J_{1}^{u}+J_{1}^{\partial^{i} u} & =\chi(\zeta)\left(\frac{1}{2 \pi i} \int \frac{\partial \varphi(\xi) / \partial \xi}{\xi-\zeta} d \xi d \xi\right) u \\
& =\chi(\zeta) \varphi(\zeta) u=\varphi(\zeta) u
\end{aligned}
$$

Similarly

$$
\partial^{i-1} J_{2}^{u}+J_{2}^{\partial^{i} u}=\partial x(\zeta) / \partial \zeta \varphi(\zeta) u=0
$$

Thus (1.2.2) combined with (1.2.3), (1.2.4) implies

$$
\left(\partial^{i-1} s^{i}+s^{i+1} \partial^{i}\right) u=[\varphi(\zeta) u]_{f}=\varphi(f) u=u .
$$

It remains only to show that $\partial^{q-1} s^{q} u=u$ if $\partial^{q} u=0, u \in A^{q}\{P[a f]\}$, however the above argument can apply also to this case with some ob- 
vious modification and the proof for this may be much simpler. The continuity of maps $s^{i}, i \leqq q$ is checked directly by inspecting (1.2.1).

Q.E.D.

Proof of Lemma 1.2.4. Set $b=1 / a$ and choose $\varphi, \chi^{\prime} \in C_{0}^{\infty}(\mathbf{C})$ so that $\chi^{\prime}=1$ in a neighbourhood of $\bar{D}_{b}, \varphi=1$ in a neighbourhood of supp $x^{\prime}$, and supp $\varphi \subset D_{1}$. Then

$$
\chi^{\prime}(\zeta) \vartheta^{i}(\varphi(f)) u /(\zeta-f) \in C_{0}^{\infty}\left(\mathbf{C}, C^{i+1}\right)
$$

for the left hand side can be written in the form

$$
\chi^{\prime}(\zeta) \partial \varphi / \partial \xi(f)\left(\partial^{i} \bar{f} u-\bar{f} \partial^{i} u\right) /(\zeta-f)
$$

which actually belongs to $C_{0}^{\infty}\left(\mathbf{C}, C^{i+1}\right)$ according to Definitions 1.1 .1 and 1.1.4. In particular, if one takes $b^{\prime}$ so that $x^{\prime}=1$ in $D_{b^{\prime}}, b<b^{\prime}<1$, then

$$
J^{u}(\zeta)=\vartheta^{i}(\varphi(f)) u /(\zeta-f) \in C^{\infty}\left(D_{b^{\prime}}, C^{i+1}\right)
$$

where the first identity is the definition of $J^{u}$. Now set for $i \geqq q$

$$
V^{u}(\zeta)=s_{2}^{i}(\varphi(f) u)-s_{2}^{i}\left((\zeta-f) s_{1}^{i+1} J^{u}(\zeta)\right)
$$

and define

$$
s^{i} u=\left[\chi(\zeta) V^{u}(\zeta)\right]_{f}
$$

where we have chosen $\chi$ so that $\chi=1$ in a neighbourhood of $\bar{D}_{b}$, supp $\chi \subset$ $D_{b^{\prime}}$. Certainly $s^{i} u \in C^{i-1}$ if $i>q ; s^{i} u \equiv 0$ modulo $B_{3}^{i-1}$ when $u \in B_{1}^{i}$, $i>q$; it is also obvious that the maps $s^{i}: C^{i} \rightarrow C^{i-1}, i>q$ and the map $s^{q}: C_{b} \rightarrow C^{\infty}\left(E^{q-1}\right)$ are continuous. If $u \in C^{i}\left\{P(f)^{c}\right\}$, then $J^{u}$ and $\varphi(f) u$ vanish identically, so that $s^{i} u=0$. Thus the first condition of Definition 1.1.3 is fulfilled when $\left(A^{\bullet}, B^{*}\right)$ replaced by $\left(B_{i}+C^{\cdot}\left\{P(f)^{c}\right\}, B_{3}^{*}+C^{\cdot}\right.$ $\left.\left\{P(a f)^{c}\right\}\right)$, so we may only prove that $\left(\partial^{i-1} s^{i}+s^{i+1} \partial^{i}\right) u \equiv u$ modulo $B_{3}^{i}+$ $C^{i}\left\{P(a f)^{c}\right\}, i \geqq q$. Using the formula (1.1.4) we obtain

$$
\partial^{i-1} s^{i} u=\left[\chi(\zeta) \partial^{i-1} V(\zeta)\right]_{f}+\vartheta^{i-1}(\bar{f})[\partial(\chi(\zeta) V(\zeta)) / \partial \zeta]_{f}
$$

since $V^{u}(\zeta)$ is analytic by (1.2.5) and (1.2.6.), the last term here equals 
$\left[\partial x(\zeta) / \partial \xi V^{u}(\zeta)\right]_{f}$ which, however, vanishes modulo $C^{i}\left\{P(a f)^{c}\right\}$ in view of the fact that $\partial x / \partial \xi(f)=0$ in a neighbourhood of $\overline{P(a f)}$. Thus

$$
\left(\partial^{i-1} s^{i}+s^{i+1} \partial^{i}\right) u \equiv\left[\chi(\zeta)\left(\partial^{i-1} V^{u}(\zeta)\right)\right]_{f} \text { modulo } C^{i}\left\{P(a f)^{c}\right\},
$$

and it suffices to show that, when $i \geqq q$,

$$
\left[\chi(\zeta)\left(\partial^{i-1} V^{u}+V^{\partial^{\imath} u}\right)(\zeta)\right]_{f} \equiv u \text { modulo } B_{3}^{i}+C^{i}\left\{P(a f)^{c}\right\}
$$

Note that, according to (1.1.2) and to the $E^{\cdot}$-analyticity of $1 /(\zeta-f)$, it holds

$$
\begin{aligned}
J^{\partial^{u} u}=\vartheta^{i+1}(\varphi(f)) \partial^{i} u /(\zeta-f) & =-\partial^{i+1} \vartheta^{i}(\varphi(f))(u /(\zeta-f)) \\
& =-\partial^{i+1} J^{u} .
\end{aligned}
$$

Hence, $s_{2}=\left(s_{2}^{i}\right)_{i \geqq q}$ being a $(q, \infty)$-homotopy of $C^{\cdot}$ modulo $\left(B_{2}^{\cdot}, B_{3}^{\cdot}\right)$, we obtain

$$
\begin{aligned}
I= & \partial^{i-1} s_{2}^{i}\left((\zeta-f) s_{1}^{i+1} J^{u}\right)+s_{2}^{i+1}\left((\zeta-f) s_{1}^{i+2} J^{\partial^{\imath} u}\right) \\
\equiv & (\zeta-f) s_{1}^{i+1} J^{u}-s_{2}^{i+1}\left((\zeta-f) \partial^{i} s_{1}^{i+1} J^{u}\right) \\
& -s_{2}^{i+1}\left((\zeta-f) s_{1}^{i+2} \partial^{i+1} J^{u}\right) \text { modulo } C^{\infty}\left(D_{b^{\prime}}, B_{3}^{i}\right)
\end{aligned}
$$

where the first identity defines $I$. Since $s_{1}=\left(s_{1}^{i}\right)_{i \geqq q}$ is a $(q, \infty)$-homotopy of $C \cdot$ modulo $\left(B_{1}, B_{2}\right)$, we have

$$
\left(\partial^{i} s_{1}^{i+1}+s_{1}^{i+2} \partial^{i+1}\right) J^{u} \equiv J^{u} \text { modulo } C^{\infty}\left(D_{b^{\prime}}, B_{2}^{i+1}\right) .
$$

This, combined with (1.2.8) and $s_{2}^{i+1}\left(B_{2}^{i+1}\right) \subset B_{3}^{i}$, gives

$$
\begin{aligned}
I & \equiv(\zeta-f) s_{1}^{i+1} J^{u}-s_{2}^{i+1}\left((\zeta-f) J^{u}\right) \\
& \equiv(\zeta-f) s_{1}^{i+1} J^{u}-s_{2}^{i+1}\left(\vartheta^{i}(\varphi(f)) u\right) \text { modulo } C^{\infty}\left(D_{b^{\prime}}, B_{3}^{i}\right)
\end{aligned}
$$

which in turn implies

$$
\begin{aligned}
& \partial^{i-1} V^{u}+V^{\partial^{i} u} \equiv \partial^{i-1} s_{2}^{i}(\varphi(f) u)+s_{2}^{i+1}\left(\varphi(f) \partial^{i} u+\vartheta^{i}(\varphi(f)) u\right) \\
&-(\zeta-f) s_{1}^{i+1} J^{u}=\left(\partial^{i-1} s_{2}^{i}+s_{2}^{i+1} \partial^{i}\right)(\varphi(f) u)-(\zeta-f) s_{1}^{i+1} J^{u} \\
& \equiv \varphi(f) u-(\zeta-f) s_{1}^{i+1} J^{u} \text { modulo } C^{\infty}\left(D_{b^{\prime}}, B_{3}^{i}\right) .
\end{aligned}
$$


Noticing $\left[(\zeta-f) s_{1}^{i+1} J^{u}\right]_{f}=0$ and by the $B_{3}$-admissibility of $f$, we thus obtain

$$
\left[\chi(\zeta)\left(\partial^{i-1} V^{u}(\zeta)+V^{\partial^{\imath} u}(\zeta)\right)\right]_{f} \equiv \chi(f) \varphi(f) u=\chi(f) u \text { modulo } B_{3}^{i} \text {. }
$$

On the other hand $u-\chi(f) u=(1-\chi(f)) u \in C^{i}\left\{P(a f)^{c}\right\}$ (i.e. $\equiv 0$ modulo $\left.C^{i}\left\{P(a f)^{c}\right\}\right)$. Thus (1.2.7) is proved.

Q.E.D.

Now we shall prove Theorems 1.2.1 and 1.2.2.

Proof of Theorem 1.2.1. When $\rho=1$, there is nothing to be proved by Lemma 1.2.3. Assuming that the conclusion is true when $\rho=k$, we shall now prove the theorem when $\rho=k+1$. Set $\tilde{f}=\left(f_{1}, \cdots, f_{\rho-1}\right)=\left(f_{1}, \cdots\right.$, $f_{k}$ ) and choose a positive constant $a^{\prime}$ so that $1<a^{\prime}<a$. By the inductive hypothesis there is a $(-\infty, q)$-homotopy $s_{1}$ for the inclusion $A^{\cdot}\{P[a \tilde{f}]\} \hookrightarrow A^{\cdot}\left\{P\left[a^{\prime} \tilde{f}\right]\right\}$ and a $(-\infty, q)$-homotopy $s_{2}$ for the inclusion $A^{\cdot}\left\{P\left[a^{\prime} \tilde{f}\right]\right\} \hookrightarrow A^{\cdot}\{P[\tilde{f}]\}$. Note here that every $A^{*}$-admissible function is also $A \cdot\{K\}$-admissible for any closed subset $K$ of $M$. Thus, applying Lemma 1.2.3 to these $s_{1}, s_{2}$, we obtain a $(-\infty, q)$-homotopy $s$ for the inclusion $A \cdot\{P[a \tilde{f}]\}\left\{P\left[a f_{k+1}\right]\right\} \hookrightarrow A \cdot\{P[\tilde{f}]\}\left\{P\left[f_{k+1}\right]\right\}$. Since $A \cdot\{P[a \tilde{f}]\}\{P$ $\left.\left[a f_{k+1}\right]\right\}=A \cdot\{P[a f]\}$ and $A \cdot\{P[\tilde{f}]\}\left\{P\left[f_{k+1}\right]\right\}=A \cdot\{P[f]\}, s$ is the desired $(q, \infty)$-homotopy.

Q.E.D.

Proof of Theorem 1.2.2. In order that the induction proceeds easily, we shall first prove the following inclusion:

$$
\sum_{i=1}^{\rho} C \cdot\left\{P\left(f_{i}\right)^{c}\right\} \subset C \cdot\left\{P(f)^{c}\right\} \subset \sum_{i=1}^{\rho} C \cdot\left\{P\left(a f_{i}\right)\right\} \quad a>1
$$

The first inclusion is obvious. To prove the second we introduce the pseudo-multiplication:

$$
g \circ h=g+h-g h \quad g, h \in C^{\infty}(M) .
$$

As is well known, this multiplication is commutative and associative, moreover $g \circ h=1$ at $z \in M$ if and only if either $g(z)=1$ or $h(z)=1$. Choose $x \in C^{\infty}(\mathbf{C})$ such that $x=1$ in a neighbourhood of the closed set 
$\mathrm{C} \backslash D_{1}$ and such that $\chi=0$ in a neighbourhood of $\bar{D}_{b}(b=1 / a)$ and set

$$
\psi=\chi\left(f_{1}\right) \circ \chi\left(f_{2}\right) \circ \cdots \circ \chi\left(f_{\rho}\right)
$$

Then $\phi=1$ in a neighbourhood of $P(f)^{c}$, hence $\phi u=u$ if $u \in C \cdot\left\{P(f)^{c}\right\}$. But $\psi$ can be written as a sum of terms $\pm \chi\left(f_{i_{1}}\right) \chi\left(f_{i_{1}}\right) \ldots \chi\left(f_{i_{p}}\right), p \leqq \rho$, and for $u \in C^{*} \pm \chi\left(f_{i_{1}}\right) \chi\left(f_{i_{2}}\right) \cdots \chi\left(f_{i_{p}}\right) u$ belongs to $C^{\cdot}$ according to the $C \cdot$-admissibility of $f_{i}, 1 \leqq i \leqq \rho$; it even belongs to $\sum_{i=1}^{\rho} C \cdot\left\{P\left(a f_{i}\right)^{c}\right\}$ because the choise of $\chi$. Thus (1.2.9) is proved.

Now we shall prove the theorem when $\rho=k+1$ assuming that the conclusion is true when $\rho=k$. Set $\tilde{f}=\left(f_{i}, \ldots, f_{\rho-1}\right)=\left(f_{1}, \ldots, f_{k}\right)$ and choose positive numbers $a^{\prime}, a^{\prime \prime}$ so that $1<a^{\prime \prime}<a^{\prime}<a$. By the inductive hypothesis we find a $(q, \infty)$-homotopy $s_{i}=\left(s_{1}^{i}\right)_{i \geqq q}$ of $C \cdot$ modulo $(C \cdot\{P$ $\left.\left.\left(a^{\prime \prime} \tilde{f}\right)^{c}\right\}, C \cdot\left\{P\left(a^{\prime} \tilde{f}\right)^{c}\right\}\right)$ and a $(q, \infty)$-homotopy $s_{2}=\left(s_{2}^{i}\right)_{i \geqq q}$ of $C^{\cdot}$ modulo $\left(C^{\cdot}\right.$ $\left.\left\{P\left(a^{\prime} \tilde{f}\right)^{c}\right\}, C \cdot\{P(a \tilde{f})\}\right)$. Applying Lemma 1.2.4 to these $s_{1}, s_{2}$ and $f=f_{\rho}$, we obtain a $(q, \infty)$-homotopy $s=\left(s^{i}\right)_{i \geqq q}$ of $C \cdot$ modulo $\left(C \cdot\left\{P\left(a^{\prime \prime} \tilde{f}\right)^{c}\right\}+\right.$ $\left.C \cdot\left\{P\left(a^{\prime \prime} f_{k+1}\right)^{c}\right\}, C \cdot\left\{P(a \tilde{f})^{c}\right\}+C \cdot\left\{P\left(a f_{k+1}\right)^{c}\right\}\right)$. But, by (1.2.9) we have

$$
\begin{aligned}
& C \cdot\left\{P\left(a^{\prime \prime} \tilde{f}\right)^{c}\right\}+C \cdot\left\{P\left(a^{\prime \prime} f_{k+1}\right)^{c}\right\} \supseteq C \cdot\left\{P(f)^{c}\right\} \\
& C \cdot\left\{P(a \tilde{f})^{c}\right\}+C \cdot\left\{P\left(a f_{k+1}\right)^{c}\right\} \subseteq C \cdot\left\{P(a f)^{c}\right\}
\end{aligned}
$$

Thus $s$ is also a $(q, \infty)$-homotopy of $C^{\cdot}$ modulo $\left(C \cdot\left\{P(f)^{c}\right\}, C \cdot\left\{P(a f)^{c}\right\}\right)$. Q.E.D.

$\S 1.3$. Consequences from the fundamental theorems. We shall start with a few corollaries which will clarify how to apply Theorems 1.2.1 and 1.2.2.

Corollary 1.3.1. Let $A$, $s$ and $f$ satisfy the hypothesis of Theorem 1.2.1. If each $A^{i}$ contains $C_{0}^{\infty}\left(E^{i}\right)$ and if $P(f)$ is relatively compact in $M$, then the sequence

$$
\cdots \rightarrow C_{0}^{\infty}\left(P(f), E^{q-1}\right) \rightarrow C_{0}^{\infty}\left(P(f), E^{q}\right) \rightarrow C_{0}^{\infty}\left(P(f), E^{q+1}\right)
$$

is exact. 
Corollary 1.3.2. Let $C \cdot s, f$ satisfy the hypothesis of Theorem 1.2.2. If each $C^{i}$ contains $C_{0}^{\infty}\left(E^{i}\right)$ and if $P\left[b_{0} f\right]$ is compact for some $0<b_{0}<1$, then the sequence

$$
\Gamma\left(P[f\lrcorner, \underline{E}^{q-1}\right) \rightarrow \Gamma\left(P\ulcorner f], \underline{E}^{q}\right) \rightarrow \Gamma\left(P[f], \underline{E}^{q+1}\right) \rightarrow \cdots
$$

is exact, where $\underline{E^{i}}, i \in \mathbf{Z}$ are the sheaves of germs of $C^{\infty}$ sections of $\underline{E}^{i}$.

Proof of Corollary 1.3.1. Suppose that $u \in C_{0}^{\infty}\left(P\left(f_{1}\right), E^{i}\right), i \leqq q$. Then there is a constant $c>1$ such that supp $u \subset P[c f]$. Choose now a constant $b$ so that $1<b<c$ and apply Theorem 1.2 .1 to $a=\frac{c}{b}$ and $f$ replaced by $b f$. Then we obtain a $(-\infty, q)$-homotopy $\sigma=\left(\sigma^{i}\right)_{i \leqq q}$ for the inclusion $A \cdot\{P[c f]\} \hookrightarrow A \cdot\{P[b f]\}$. Note $u \in A_{i}\{P[c f]\}$ (because $u \in$ $C_{0}^{\infty}\left(E^{i}\right) \subset A^{i}$ and supp $\left.u \subset P[c f]\right)$. Thus $\sigma^{i} u$ is well defined and lies in $A^{i-1}\{P[b f]\} \subset C_{0}^{\infty}\left(P(f), E^{i-1}\right)$, moreover $\partial^{i-1} \sigma^{i} u=u$ if $\partial^{i} u=0$. Thus the exactness of (1.3.1) is proved.

Proof of Corollary 1.3.2. Suppose that $\left.u \in \Gamma\left(P[f], \underline{E}^{i}\right)\right), i \leqq q$ satisfies the equation $\partial^{i} u=0$. Then there is a constant $b_{1}$ such that $b_{0}<b_{1}<1$, and an element $v \in C^{\infty}\left(P\left(b_{1} f\right), E^{i}\right)$ which, when restricted to $P[f]$, induces $u$ and still satisfies $\partial^{i} v=0$. Choose $b_{2}, b_{3}$ so that $b_{1}<b_{2}<b_{3}<1$ and choose $\varphi \in C_{0}^{\infty}\left(P\left(b_{1} f\right)\right)$ such that $\varphi=1$ in $P\left(b_{2} f\right)$. Then $\varphi v \in C_{0}^{\infty}\left(E^{i}\right)$ $C C^{i}$ and $\partial^{i}(\varphi v) \in C^{i+1}\left\{P\left(b_{2} f\right)^{c}\right\}$. Now apply Theorem 1.2 .2 for $a=b_{3} / b_{2}$ and for $f$ replaced by $b_{2} f$, then we find a $(q, \infty)$-homotopy $\sigma=\left(\sigma^{i}\right)_{i \geqq q}$ of $C \cdot$ modulo $\left(C \cdot\left\{P\left(b_{2} f\right)^{c}\right\}, C \cdot\left\{P\left(b_{3} f\right)^{c}\right\}\right)$. Thus, by Definition 1.1.3, we have

$$
\begin{gathered}
\sigma^{i+1} \partial^{i}(\varphi v) \in C^{i}\left\{P\left(b_{3} f\right)^{c}\right\} \\
\partial^{i-1} \sigma^{i}(\varphi v)+\sigma^{i+1} \partial^{i}(\varphi v)-\varphi v \in C^{i}\left\{P\left(b_{3} f\right)^{c}\right\}
\end{gathered}
$$

from which it follows

$$
\partial^{i-1}\left(\sigma^{i}(\varphi v) \mid P\left(b_{3} f\right)\right)=\varphi v\left|P\left(b_{3} f\right)=v\right| P\left(b_{3} f\right) .
$$

Thus, if one denotes by $w$ the element of $\Gamma\left(P[f], \underline{E}^{i-1}\right)$ induced by $\sigma^{i}(\varphi v)$, then $\partial^{i-1} w=u$, which proves the exactness of (1.3.2). 
We shall now explain some of the further implications of Corollaries 1.3.1 and 1.3.2. Let $A{ }^{\circ}, s$ satisfy the hypothesis of Theorem 1.2 .1 and assume $C_{0}^{\infty}\left(E^{\cdot}\right) \subset A^{\cdot}$. Assume further that there is a sequence of positive integers $\rho_{\nu}, \nu=1,2, \ldots$ and a sequence $f^{\nu}=\left(f_{1}^{\nu}, f_{2}^{\nu}, \ldots, f_{\rho_{\nu}}^{\nu}\right) \nu=1,2, \ldots$, such that each $f_{k}^{\nu} 1 \leqq k \leqq \sigma_{\nu}, \nu=1,2, \ldots$ is $E^{*}$-analytic and $A^{*}$-admissible. If $P\left[f^{\nu}\right]$ are compact, $P\left[f^{\nu}\right] \subset P\left(f^{\nu+1}\right)$ and if $M=\bigcup_{\nu} P\left(f^{\nu}\right)$, then the sequence

$$
\cdots \rightarrow C_{0}^{\infty}\left(E^{q-1}\right) \rightarrow C_{0}^{\infty}\left(E^{q}\right) \rightarrow C_{0}^{\infty}\left(E^{q+1}\right)
$$

is exact. This is an immediate consequence from Corollary 1.3.1.

Remark. In Corollary 1.3.2 we may assume only the compactness of $P[f]$ instead of assuming that $P\left[b_{0} f\right]$ is compact for some $0<b_{0}<1$; for, if $P[f]$ is compact, then, for every neighbourhood of $P[f]$ there is some $0<b_{0}<1$ such that any component of $P\left[b_{0} f\right]$ which intersects $P[f]$ is necessarily contained in this neighbourhood.

Let now $C \cdot s$ satisfy the hypothesis of Theorem 1.2.2 and assume $C_{0}^{\infty}\left(E^{*}\right) \subset C^{*}$. Assume further that there is a sequence of positive integers $\rho_{\nu}, \nu=1,2, \ldots$ and a sequence $f^{\nu}=\left(f_{1}^{\nu}, \ldots, f_{\rho_{\nu}}^{\nu}\right)$ such that each $f_{k}^{\nu}, 1 \leqq k$ $\leqq \rho_{\nu} ; \nu=1,2, \ldots$ is $E^{\cdot}$-analytic and $C^{\cdot}$-admissible. If $P\left[f^{\nu}\right]$ are compact, $P\left[f^{\nu}\right] \subset P\left(f^{\nu+1}\right)$ (resp. $P\left[f^{\nu+1}\right] \subset P\left(f^{\nu}\right)$ ) and if $M=\bigcup_{\nu} P\left(f^{\nu}\right)$ (resp. $\bigcap_{\nu} P\left(f_{\nu}\right)=\left\{z_{0}\right\}$ for a point $\left.z_{0} \in M\right)$, then the sequence

$$
\begin{aligned}
& C^{\infty}\left(E^{q}\right) \rightarrow C^{\infty}\left(E^{q+1}\right) \rightarrow \cdots \\
& \underline{E}_{z_{0}}^{q-1} \rightarrow \underline{E}_{z_{0}}^{q} \rightarrow \underline{E}_{z_{0}}^{q+1} \rightarrow \cdots
\end{aligned}
$$

is exact. In case some approximation theorem holds for solutions of the equation $\partial^{q-1} u=0$ in the domain of the form $P(f)$, the exact sequence (1.3.4) can be slightly extentded, for example, if every solution of $\partial^{q-1} u$ $=0$ in $P\left(f_{\nu}\right)$ can be approximated by global solutions of $\partial^{q-1} u=0$ in the $C^{\infty}$ topology, then the sequence

$$
C^{\infty}\left(E^{q-1}\right) \rightarrow C^{\infty}\left(E^{q}\right) \rightarrow C^{\infty}\left(E^{q+1}\right) \rightarrow \cdots
$$


is exact. The exactness of (1.3.5) follows directly from Corollary 1.3.2. The proof of the exactness of (1.3.4) and of $(1.3 .4)^{\prime}$ is routine and omitted.

Now we shall define the dual complex $F^{\cdot}$ of $E^{\cdot}$ and prove some lemmas which indicate what can deduced, on the side of the dual $F^{\text {. }}$ from the conclusions of Theorems 1.2.1 and 1.2.2. Let $\Theta$ be the line bundle of (complex-valued) $n$-forms on $M\left(n=\operatorname{dim}_{\mathbf{R}} M\right)$ and set $F^{i}=\left(E^{-i}\right)^{*}$ $\otimes \Theta$ where $E^{i *}$ denotes the dual vector bundle of $E^{i}$. Denote by $\langle 1\rangle$ the canonical duality homomorphism from $E^{-i} \otimes F^{i}$ onto $\Theta$. Then there is a unique differential operator $\partial^{* i}: F^{i} \rightarrow F^{i+1}$ such that

$$
\int\left\langle u \mid \partial^{* i} v\right\rangle=\int\left\langle\partial^{-i-1} u \mid v\right\rangle \quad u \in C_{0}^{\infty}\left(E^{-i-1}\right), v \in C_{0}^{\infty}\left(F^{i}\right)
$$

where we have assumed that $M$ is oriented and the integration is taken over the orientation of $M$. Obvionsly $\partial^{*(i+1)} \partial^{* i}=0$ and the complex

$$
\cdots \longrightarrow F^{i-1} \stackrel{\partial *(i-1)}{\longrightarrow} F^{i} \stackrel{\partial^{* i}}{\longrightarrow} F^{i+1} \longrightarrow \cdots
$$

is called the dual complex of $E$. To state the lemmas we need a notation: $\mathscr{D}^{\prime}(U, F)$, where $F$ is a vector bundle over $M$ and $U$ is an open subset of $M$, denotes the set of all sections of $F$ with coefficients in the space of distributions on $M$, whereas $\mathscr{E}^{\prime}(U, F)$ denotes the subspace of $\mathscr{D}^{\prime}(U, F)$ of all elements with compact support.

Lemma 1.3.3. Let $K, K^{\prime}$ be two closed subsets of $M$ such that $K C$ $K^{\prime}$ and let $\Omega$ be the interior of $K$. Let further $A$ be a subcomplex such

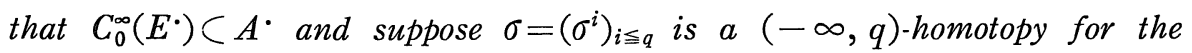
inclusion $A \cdot\{K\} \hookrightarrow A \cdot\left\{K^{\prime}\right\}$.

If $u$ is a distribution section of $E^{i}(i>-q)$ defined over a neighbourhood of $K^{\prime}$ satisfying the equation $\partial^{* i} u=0$, then one can find $v \in$ $\mathscr{D}^{\prime}(\Omega, F)$ such that $\partial^{*(i-1)} v=u \mid \Omega$.

Proof. On the subspace $\left\{\partial^{-i} w \mid w \in C_{0}^{\infty}\left(\Omega, E^{-i}\right)\right\}$ of $C_{0}^{\infty}\left(\Omega, E^{-i+1}\right)$ we define a linear form $l$ by

$$
l\left(\partial^{-i} w\right)=\int\langle w \mid u\rangle .
$$


This is actually well defined and continuous since

$$
\begin{aligned}
\int\langle w \mid u\rangle & =\int\left\langle\partial^{-i-1} \sigma^{-i} w \mid u\right\rangle+\int\left\langle\sigma^{-i+1} \partial^{-i} w \mid u\right\rangle \\
& =\int\left\langle\sigma^{-i} w \mid \partial^{* i} u\right\rangle+\int\left\langle\sigma^{-i+1} \partial^{-i} w \mid u\right\rangle \\
& =\int\left\langle\sigma^{-i+1} \partial^{-i} w \mid u\right\rangle .
\end{aligned}
$$

Thus, by the Hahn-Banach theorem, there is $v \in \mathscr{D}^{\prime}\left(\Omega, F^{i-1}\right)$ such that $l\left(\partial^{-i} w\right)=\int\left\langle\partial^{-i} w \mid v\right\rangle$, hence $\int\left\langle\partial^{-i} w \mid v\right\rangle=\int\langle w \mid u\rangle$, for $w \in C_{0}^{\infty}\left(\Omega, E^{-i}\right)$, that is, $\partial^{*(i-1)} v=u \mid \Omega$.

Q.E.D.

By the similar reasoning we can prove

Lemma 1.3.4. Let $\Omega, \Omega^{\prime}$ be open subsets of $M$ such that $\bar{\Omega} \hookrightarrow \Omega^{\prime}$. Suppose that $C^{\cdot}$ is a subocmplex of $C^{\infty}\left(E^{i}\right)$ such that $C_{0}^{\infty}\left(E^{*}\right) \subset C^{\cdot}$, and that $\sigma=\left(\sigma^{i}\right)_{i \geqq q}$ is a $(q, \infty)$-homotopy of $C^{\cdot}$ modulo $\left(C^{\circ} \cdot\left\{\left(\Omega^{\prime}\right)^{c}\right\}, C \cdot\left\{\Omega^{c}\right\}\right)$. If $u \in \mathscr{E}^{\prime}\left(\Omega, F^{i}\right), i<-q$ satisfies $\partial^{* i} u=0$, then one can find $v \in \mathscr{E}^{\prime}\left(\Omega^{\prime}\right.$, $\left.F^{i-1}\right)$ such that $\partial^{*(i-1)} v=u$.

Let $\mathscr{D}^{\prime} F^{i}, i \in \mathbf{Z}$ denote the sheaf of germs of distribution sections of $F^{i}$. Using Lemmas 1.3 .3 and 1.3 .4 we can easily deduce from Theorems 1.2.1 and 1.2.2, the following corollaries.

Corollary 1.3.5. Let $A^{\circ}, s, f$ satisfy the hypothesis of Theorem 1.2.1. If $C_{0}^{\infty}\left(E^{\cdot}\right) \subset A^{\cdot}$ and if $P\left[b_{0} f\right]$ is compact for some $0<b_{0}<1$, then the sequence

$$
\Gamma\left(P[f], \mathscr{D}^{\prime} F^{-q}\right) \rightarrow \Gamma\left(P[f], \mathscr{D}^{\prime} F^{-q+1}\right) \rightarrow \cdots
$$

is exact.

Corollary 1.3.6. Let $C \cdot, s, f$ satisfy the hypothesis of Theorem 1.2.2. If $C_{0}^{\infty}\left(E^{\cdot}\right) \subset C^{\cdot}$ and if $P(f)$ is relatively compact in $M$, then the sequence

$$
\cdots \rightarrow \mathscr{E}^{\prime}\left(P(f), F^{-q-1}\right) \rightarrow \mathscr{E}^{\prime}\left(P(f), F^{-q}\right)
$$


is exact.

§1.4. Suitable estimation. In this section we present a method to derive from certain estimates concerning some Dirichlet norms for $E^{*}$, suitable subcomplexes of $C^{\infty}\left(E^{\cdot}\right)$ as well as some related partial homotopies to which Theorems 1.2.1 and 1.2.2 can be applied. To fix the Dirichlet norms we choose a volume element $d v$ and Hermitian inner products $\langle,\rangle_{i}$ for the vector bundles $E^{i}$, and we set

$$
\begin{aligned}
(u, v)_{i} & =\int\langle u, v\rangle_{i} d v \quad u, v \in C_{0}^{\infty}\left(E^{i}\right) \\
\|u\|_{i}^{2} & =\int\langle u, u\rangle_{i} d v=(u, u)_{i} .
\end{aligned}
$$

Further, we define the formal adjoint $\delta^{i}: E^{i} \rightarrow E^{i-1}$ of the differential operator $\partial^{i-1}: E^{i-1} \rightarrow E^{i}$ by setting

$$
\left(u, \delta^{i} v\right)=\left(\partial^{i-1} u, v\right) \quad u \in C_{0}^{\infty}\left(E^{i-1}\right), v \in C_{0}^{\infty}\left(E^{i}\right)
$$

Then the square root of

$$
D_{1}(u)=\left\|\partial^{i} u\right\|_{i+1}^{2}+\left\|\delta^{i} u\right\|_{i-1}^{2} \quad u \in C_{0}^{\infty}\left(E^{i}\right)
$$

is the so-called Dirichlet norm for the complex $E^{\cdot}$ (with respect to inner products $\left\langle,>_{i}, i \in Z\right.$ and volume $\left.d v\right)$. By completing $C_{0}^{\infty}\left(E^{i}\right)$ by the norm \|\|$_{i}$ we define the Hilbert space $H^{i}$. As is well known, $H^{i}$ can also be regarded as the space of all locally square integrable sections $u$ of $E^{i}$ such that $\|u\|_{i}<+\infty$. (Here we shall of course extend the norm \|\|$_{i}$ to any locally square integrable sections of $E^{i}$ allowing $\infty$ to be one of the values of this norm.) The differential operators $\partial^{i}, \delta^{i}$ give rise to linear, closed, densely defined operators $T^{i}: H^{i} \rightarrow H^{i+1}, S^{i}: H^{i}$ $\rightarrow H^{i-1}$; an element $u \in H^{i}$ is in the domain $D\left(T^{i}\right)$ of $T^{i}$ (resp. $D\left(S^{i}\right)$ of $S^{i}$ ) if and only if $\partial^{i} u$ (resp. $\delta^{i} u$ ), defined in the distribution sense, belongs to $H^{i+1}$ (resp. $H^{i-1}$ ). The adjoint of $T^{i-1}$ does not coincide with $S^{i}$ in general. To guarantee the identity of these we shall impose the following assumption for each $i \in Z$ : 
(*) There is a sequence $\eta_{\nu} \in C_{0}^{\infty}(M)$ such that (1) for any compact $K \subset M, \eta_{\nu}=1$ in $K$ when $\nu$ sufficiently large, (2) the inequality

$$
\left|\vartheta^{i}\left(\eta_{\nu}\right) u\right|_{i+1} \leqq C|u|_{i} \quad u \in E^{i}
$$

is valid for some constant $C$ not depending on $\nu$ and $u$.

Lemma 1.4.1 (Hörmander [4]). Let the assumption (*) be fulfilled. Then $C_{0}^{\infty}\left(E^{i}\right)$ is dense in $D\left(T^{i}\right) \cap D\left(S^{i}\right)$ for the norm $\|u\|_{i}+D_{i}(u)^{\frac{1}{2}}$. In particular $S^{i}=\left(T^{i-1}\right)^{*}$.

Here we have considered the Dirichlet norm $D_{i}(u)^{\frac{1}{2}}$ to be extended over $D\left(T^{i}\right) \cap D\left(S^{i}\right)$.

We shall now study some consequence of the estimates of the form $\|u\|_{i}^{2} \leqq C D_{i}(u) u \in C_{0}^{\infty}\left(E^{i}\right)$. We first refer to a result of Hörmander [4] which is fundamental in the subsequent study: Denote by $N(T), R(T)$, the null space and the range of an operator $T$ from one Hilbert space into another.

Theorem 1.4.2 ([4]). Let $\left(^{*}\right)$ be fulfilled and assume that the estimate

$$
\|u\|_{i}^{2} \leqq C D_{i}(u) \quad u \in C_{0}^{\infty}\left(E^{i}\right)
$$

is valid for some constant $C>0$. Then $R\left(T^{i-1}\right)=N\left(T^{i}\right)$ and $N\left(S^{i}\right)=$ $R\left(S^{i+1}\right)$. In particular $R\left(T^{i}\right), R\left(T^{i-1}\right), R\left(S^{i}\right), R\left(S^{i+1}\right)$ are closed.

If $R\left(T^{i}\right)$ is closed, then there is a unique bounded operator $Q^{i+1}: H^{i+1}$ $\rightarrow H^{i}$ with the following properties:

(i) $R\left(Q^{i+1}\right) \subseteq N\left(T^{i}\right)^{\perp} \cap D\left(T^{i}\right)$

(ii) $1_{H^{i+1}}-T^{i} Q^{i+1}$ is the orthogonal projection of $H^{i+1}$ onto $R\left(T^{i}\right)^{\perp}$. Here we have used the notation $L^{\perp}$ to express the orthogonal complement in $H$ when $L$ is a subspace of a Hilbert space $H$. From Theorem 1.4.2 we obtain the following lemma which is useful later.

Corollary 1.4.3. Let the hypothesis of Theorem 1.4 .2 be fulfilled. Then the operators $Q^{i}, Q^{i+1}$ above are well defined and it holds 


$$
\left(T^{i-1} Q^{i}+Q^{i+1} T^{i}\right) u=u \quad u \in D\left(T^{i}\right) .
$$

Moreover, if the differential operator $\Delta^{i}=\partial^{i-1} \delta^{i}+\delta^{i+1} \partial^{i}$ is hypoelliptic, then $Q^{i} u\left(u \in H^{i}\right)$ is smooth whenever $u$ is smooth.

Proof. The first half is obvious. To prove the second half, note that the estimate (1.4.1) implies

$$
\|u\|_{i} \leqq C\left\|\Delta^{i} u\right\|_{i} \quad u \in C_{0}^{\infty}\left(E^{i}\right)
$$

Thus, $R\left(\Lambda^{i}\right)=H^{i}$, if one defines the operator $\Lambda^{i}=T^{i-1} S^{i}+S^{i+1} T^{i}$ by setting $D\left(\Lambda^{i}\right)=\left\{u \in D\left(S^{i}\right) \cap D\left(T^{i}\right) \mid S^{i} u \in D\left(T^{i-1}\right), \quad T^{i} u \in D\left(S^{i+1}\right)\right\} . \quad$ By the hypoellipticity of $\Delta^{i}, \Lambda^{i} v$ is smooth if and only if $v \in D\left(\Lambda^{i}\right)$ is smooth. Since $Q^{i} \Lambda^{i} v=S^{i} v v \in D\left(\Lambda^{i}\right)$ by the definition of $Q^{i}, Q^{i} u$ is smooth whenever $u$ is smooth.

Q.E.D.

With the application of Theorems 1.2.1 and 1.2.2 in mind we will assume one of the following assumptions, frequently in the sequel:

$\left(\mathscr{A}_{q}\right)$ The estimate (1.4.1) is valid for $i \leqq q$, the differential operator $\Delta^{i}$ is hypoelliptic when $i \leqq q$,

$\left(\mathscr{B}_{q}\right)$ The estimate (1.4.1) is valid for $i \geqq q$, the differential operator $\Delta^{i}$ is hypoelliptic when $i \geqq q$.

According to Lemma 1.4 .3 the assumption $\left(\mathscr{A}_{q}\right)$ (resp. $\left(\mathscr{B}_{q}\right)$ ) gives a sequence of operators $\left(Q^{i}\right)_{i \leqq q}\left(\right.$ resp. $\left.\left(Q^{i}\right)_{i \geqq q}\right)$ for which (1.4.2) is valid; thus, if in addition one can choose a suitable subcomplex $A^{\cdot}$ of $C^{\infty}\left(F^{\cdot}\right)$ so that $O^{i}\left(A^{i}\right) \subset A^{i-1}, Q^{i} \mid A^{i}: A^{i} \rightarrow A^{i-1}$ is continuous when $i \leqq q$ (resp. $i>q)$, then we obtain a $(-\infty, q)$-homotopy $s=\left(s^{i}\right)_{i \leqq q}$ for $A^{\bullet} \hookrightarrow A$. (resp. $(q, \infty)$-homotopy $s=\left(s^{i}\right)_{i \geqq q}$ of $\left.A^{\cdot}\right)$ setting $s^{i}=Q^{i} \mid A^{i} i \leqq q$ (resp. $1 \geqq q$ ). But a question still remains. How rich is the set of all functions which are $E^{\cdot}$-analytic and $A^{\circ}$-admissible? The answer is in fact indispensable when applying Theorems 1.2.1 and 1.2.2. Instead of solving this difficult problem we adopt here the inverse process: First we choose a subring $R$ of $A\left(E^{*}\right)$ (for this notation see $\S 1.1$ ) with certain good properties; then we find a subcomplex $A_{R}^{\cdot}$ of $C^{\infty}\left(E^{*}\right)$ so that $Q^{i}\left(A_{R}^{i}\right) \subset A_{R}^{i-1}, i \leqq q$ (resp. 
$i>q)$ if $\left(\mathscr{A}_{q}\right)$ (resp. $\left.\left(\mathscr{B}_{q}\right)\right)$ is fulfilled, and so that any $f \in R$ is $A_{R}^{\text {-admis- }}$ sible.

Definition 1.4.4. A subring $R$ of $A\left(E^{*}\right)$ is said to be well filtered if and only if there is an ascending chain of linear subspaces of $R R_{0} C$ $R_{1} \subset R_{2} \subset \cdots$ and a sequence of elements of $C^{\infty}(M) \chi_{\nu}, \nu=1,2, \ldots$ satisfying the following conditions:

(i) $\quad R_{0}=\mathrm{C}, \quad R=\bigcup_{k} R_{k}$.

(ii) For any $i \in Z$ and for every element $f \in R_{k}$ one can find a finite number of elements of $R_{k-1} g_{j}, j=1,2, \ldots, s_{f}$ so that the estimate

$$
\left|\vartheta^{i}(\bar{f}) u\right|_{i+1} \leqq \sum_{j=1}^{s_{f}}\left|g_{j} u\right|_{i} \quad u \in E^{i}
$$

holds.

(iii) $0 \leqq x_{\nu} \leqq 1, x_{\nu} \rightarrow 1(\nu \rightarrow \infty)$ in the $C^{\infty}$-topology, $x_{\nu} f$ is bounded for any $f \in R$.

(iv) For $i \in \mathbf{Z}$ there is a sequence of constants $C_{\nu} \nu=1,2, \ldots$ tending to 0 as $\nu \rightarrow \infty$, for which the estimate

$$
\left|\vartheta^{i}\left(\chi_{\nu}\right) u\right|_{i+1} \leqq C_{\nu}\left|\chi_{\nu} u\right|_{i} \quad u \in E^{i}
$$

holds.

Now we set for a given subring $R$ of $A\left(E^{*}\right)$

$$
A_{R}^{i}=\left\{u \in C^{\infty}\left(E^{i}\right) \mid g u \in H^{i}, g \partial^{i} u \in H^{i+1} \text { for all } g \in R\right\}
$$

and define the locally convex topology of $A_{R}^{i}$ by adding seminorms $\|g u\|_{\imath}$, $\left\|g \partial^{i} u\right\|_{i+1}$, where $g$ runs over $R$, to the seminorms defining the topology of $C^{\infty}\left(E^{i}\right)$. Obviously $A_{R}^{i}, i \in \mathbb{Z}$ are complete and $\partial^{i}\left(A_{R}^{i}\right) \subset A_{R}^{i+1}, \partial^{i} \mid A_{R}^{i}: A_{R}^{i} \rightarrow$ $A_{R}^{i+1}$ is continuous, thus the complex $A_{R}=\cdots \rightarrow A_{R}^{i-1} \rightarrow A_{R}^{i} \rightarrow A_{R}^{i+1} \rightarrow \cdots$ is a complete subcomplex of $C^{\infty}\left(E^{*}\right)$.

Theorem 1.4.5. Let the notation be as above. If a subring $R$ is well filtered, then every $f \in R$ is $A_{R^{-}}$admissible. If moreover the assump- 
tions $\left(^{*}\right)$ and $\left(\mathscr{A}_{q}\right)\left(\right.$ resp. $\left.\left(\mathscr{B}_{q}\right)\right)$ are satisfied, then $Q^{i}\left(A_{R}^{i}\right) \subset A_{R}^{i-1}, Q^{i} \mid A_{R}^{i}: A_{R}^{i}$ $\rightarrow A_{R}^{i-1}$ is continuous for $i \leqq q$ (resp. $i>q$ ), so that $s=\left(s^{i}\right)_{i \leqq q}$ (resp. $s=$ $\left.\left(s^{i}\right)_{i \geqq q}\right)$ is a $(-\infty, q)$-homotopy for $A_{R} \rightarrow A_{R}$ (resp. $(q, \infty)$-homotopy of $\left.A_{R}^{*}\right)$ if one defines $s^{i}: A_{R}^{i} \rightarrow A_{R}^{i-1}, i \leqq q$ (resp. $s^{i}: A_{R}^{i} \rightarrow A_{R}^{i-1} i>q$ and $s^{q}: A^{q} \rightarrow$ $\left.C^{\infty}\left(E^{q-1}\right)\right)$ as the restrictions of $Q^{i}$ to $A_{R}^{i}$.

The proof we divide into parts:

Proof of the first half of Theorem 1.4.5. We first prove the condition (1) of Definition 1.1.4. That $f u \in A_{R}^{i}$ for $f \in R, u \in A_{R}^{i}$ and the continuity of map $A_{R}^{i} \ni u \mapsto f u \in A_{R}^{i}$ follow immediately from the definition of $A_{R}^{i}$. Let us prove $\bar{f} u \in A_{R}^{i}$ for $f \in R, u \in A_{R}^{i}$. Let $f \in R_{k}$ and let $g_{j}$ $j=1,2, \cdots, s_{f}$ of $R_{k-1}$ satisfy (1.4.3). Then, for any $g \in R$,

$$
\begin{aligned}
\left\|g \partial^{i}(\bar{f} u)\right\|_{i+1} & \leqq\left\|\bar{f} g \partial^{i} u\right\|_{i+1}+\left\|g \vartheta^{i}(\bar{f}) u\right\|_{i+1} \\
& \leqq\left\|f g \partial^{i} u\right\|_{i+1}+\sum_{j=1}^{s f}\left\|g g_{j} u\right\|_{i}<+\infty .
\end{aligned}
$$

Since obviously $\|g \bar{f} u\|_{i}=\|f g u\|_{i}<+\infty$, we have thus proved $\bar{f} u \in A_{R}^{i}$. The map $A_{R}^{i} \ni u \rightarrow \bar{f} u \in A_{R}^{i}$ is evidently continuous. Note that

$$
\vartheta^{i}(\varphi(f))=\frac{\partial \varphi}{\partial \zeta}(f) \vartheta^{i}(f)+\frac{\partial \varphi}{\partial \bar{\zeta}} \vartheta^{i}(\bar{f})=\frac{\partial \varphi}{\partial \bar{\zeta}} \vartheta^{i}(\bar{f})
$$

for $\varphi(\zeta) \in C_{0}^{\infty}(C)$ and for $f \in A\left(E^{*}\right)$. Since $\varphi, \partial \varphi / \partial \zeta$ are bounded, we obtain also by using (1.4.3) $\|g \varphi(f) u\|_{i}<+\infty,\left\|g \partial^{i}(\varphi(f) u)\right\|_{i+1}<+\infty$ if $u \in A_{R}^{i}$ and $f, g \in R$. Thus $\varphi(f) u \in A_{R}^{i}$. The continuity of map $A_{R}^{i} \ni u$ $\rightarrow \varphi(f) u \in A_{R}^{i}(f \in R)$ is also evident. The condition (2) of Definition 1.1.4 can be proved similarly.

To prove the condition (3) we need a formula: Let $V(\zeta, z) \in C_{0}^{\infty}$ $\left(\mathrm{C}, C_{0}^{\infty}\left(E^{i}\right)\right)=C_{0}^{\infty}\left(C \times M, \tilde{E}^{i}\right)\left(\tilde{E}^{i}\right.$ is the pull back of $E^{i}$ by the projection $\mathrm{C} \times M \rightarrow M)$, and let $\zeta=x+\sqrt{-1} y$. Then

$$
\begin{aligned}
& \left|\frac{\partial}{\partial x}<V, V>_{i}\right|=2\left|\operatorname{Re}<\frac{\partial V}{\partial x}, V>_{i}\right| \leqq|V|_{i}^{2}+\left|\frac{\partial V}{\partial x}\right|_{i}^{2} \\
& \left|\frac{\partial}{\partial y}<V, V>_{i}\right| \leqq|V|_{i}^{2}+\left|\frac{\partial V}{\partial y}\right|_{i}^{2}
\end{aligned}
$$


Hence we obtain for any $(\zeta, z) \in \mathbb{C} \times M$

$$
\begin{aligned}
|V(\zeta, z)|_{i}^{2} \leqq & \int\left(|V(x+\sqrt{-1} y, z)|_{i}^{2}+\left|\frac{\partial V}{\partial x}(x+\sqrt{-1} y, z)\right|_{i}^{2}\right. \\
& \left.+\left|\frac{\partial V}{\partial y}(x+\sqrt{-1} y, z)\right|_{i}^{2}+\left|\frac{\partial^{2} V}{\partial x \partial y}(x+\sqrt{-1} y, z)\right|_{i}^{2}\right) d x d y .
\end{aligned}
$$

Thus we have finally

$$
\begin{aligned}
\left\|[V]_{f}\right\|_{i}^{2} \leqq \int\left(\|V(x+\sqrt{-1} y)\|_{i}^{2}+\left\|\frac{\partial V}{\partial x}(x+\sqrt{-1} y)\right\|_{i}^{2}\right. \\
\left.+\left\|\frac{\partial V}{\partial y}(x+\sqrt{-1} y)\right\|_{i}^{2}+\left\|\frac{\partial^{2} V}{\partial x \partial y}(x+\sqrt{-1} y)\right\|_{i}\right) d x d y .
\end{aligned}
$$

Now suppose $f \in R, V \in C_{0}^{\infty}\left(\mathbb{C}, A_{R}^{i}\right)$. We shall prove $[V]_{f} \in A_{R}^{i}$. Note that the proof of (1) of Definition 1.1 .4 gives $g V \in C_{0}^{\infty}\left(\mathbb{C}, A_{R}^{i}\right)$ if $g \in R$. Thus, applying (1.4.5) to $V$ replaced by $g V$, we obtain

$$
\left\|g[V]_{f}\right\|=\left\|[g V]_{f}\right\|<+\infty \quad g \in R .
$$

Note

$$
g \partial^{i}[V]_{f}=\left[g \partial^{i} V\right]_{f}+\left[\vartheta^{i}(\bar{f}) g \frac{\partial V}{\partial \bar{\zeta}}\right]_{f},
$$

where both $g \partial^{i} V, \vartheta^{i}(\bar{f}) g \frac{\partial V}{\partial \bar{\zeta}}$ belongs $C_{0}^{\infty}\left(\mathbf{C}, A_{R}^{i+1}\right)$ as we have proved above. Applying (1.4.5) to these and to $i$ replaced by $i+1$, we conclude

$$
\left\|g \partial^{i}[V]_{f}\right\|_{i+1}<+\infty \text {. }
$$

This, combined with (1.4.6), proves $[V]_{f} \in A_{P}^{i}$. The map $C_{0}^{\infty}\left(\mathbb{C}, A_{R}^{i}\right) \in V$ $\rightarrow[V]_{f} \in A_{R}^{i}$ is evidently continuous. (The condition (4) of Definition 1.1.4 was also proved above.)

Q.E.D.

Remark. In this proof we have not used $\chi_{\nu}$ and the requirement $g_{j}$ are elements of $R_{k-1}$, in fact what we have needed is only $g_{j} \in R$. (But, nothing is gained by this improvement.) 
To prove the second half of Theorem 1.4.5, we require the following Lemma.

Lemma 1.4.6. Assume (*) and suppose that the estimate (1.4.1) is valid and that a subring $R$ is well filtered. Let $f \in R, u \in H^{i} \cap C^{\infty}\left(E^{i}\right)$, $\delta^{i}(f u) \in H^{i-1}$, and $\partial^{i}(f u) \in H^{i+1}$. Then $f u \in H^{i}$, hence $f u \in D\left(T^{i}\right) \cap$ $D\left(S^{i}\right)$ and

$$
\|f u\|_{i}^{2} \leqq D_{i}(f u)
$$

Proof. Let $R_{0} \subset R_{1} \subset R_{2} \subset \cdots$ and $\chi_{\nu}$ satisfy the conditions of Definition 1.4.4. Since $x_{\nu} f$ is bounded, we have $x_{\nu} f u \in H^{i}$. From (1.4.4) and from the formulas

$$
\begin{aligned}
& \partial^{i}\left(x_{\nu} f u\right)=\vartheta^{i}\left(x_{\nu}\right) f u+x_{\nu} \partial^{i}(f u) \\
& \delta^{i}\left(x_{\nu} f u\right)=\vartheta^{i-1}\left(x_{\nu}\right) * f u+x_{\nu} \delta^{i}(f u)
\end{aligned}
$$

it follows that $\partial^{i}\left(x_{\nu} f u\right) \in H^{i+1}, \delta^{i}\left(x_{\nu} f u\right) \in H^{i-1}$, and that

$$
\begin{aligned}
& \left\|\partial^{i}\left(x_{\nu} f u\right)\right\|_{i+1} \leqq\left\|\partial^{i} f u\right\|_{i+1}+C_{\nu}\left\|x_{\nu} f u\right\|_{i} \\
& \left\|\delta^{i}\left(x_{\nu} f u\right)\right\|_{i+1} \leqq\left\|\delta^{i} f u\right\|_{i-1}+C_{\nu}\left\|x_{\nu} f u\right\|_{i} .
\end{aligned}
$$

Thus, in view of Lemma 1.4.1, $x_{\nu} f u \in D\left(T^{i}\right) \cap D\left(S^{i}\right)$. Applying (1.4.1) to $x_{\nu} f u$ we obtain

$$
\left\|x_{\nu} f u\right\|_{i}^{2} \leqq 2 C\left(D_{i}(f u)+C_{\nu}^{2}\left\|x_{\nu} f u\right\|_{i}^{2}\right)
$$

Hence, for $\nu$ so large that $2 C C_{\nu}^{2} \leqq \frac{1}{2}$, we have

$$
\left\|x_{\nu} f u\right\|_{i}^{2} \leqq 4 C D_{i}(f u)
$$

Note that the right hand side does not depend on $\nu$, thus by the Fatou Lemma we obtain

$$
\|f u\|_{i}^{2} \leqq \varlimsup_{\nu \rightarrow \infty}\left\|x_{\nu} f u\right\|_{i}^{2}<+\infty
$$

That is, $f u \in H^{i} . \quad$ According to Lemma 1.4.1 this implies $f u \in D\left(T^{i}\right) \cap$ 
$D\left(S^{i}\right)$, hence

$$
\|f u\|_{i}^{2} \leqq D_{i}(f u)
$$

Q.E.D.

End of the proof of Theorem 1.4.5. To prove the last half of Theorem 1.4.5 it suffices to prove that $Q^{l}\left(A_{R}^{l}\right) \subset A_{R}^{l-1}, Q^{l} \mid A_{R}^{l}: A_{R}^{l} \rightarrow A_{R}^{l-1}$ is continuous provided that (1.4.1) is valid for $i=l$ and for $i=l-1$, and that $\Delta^{l-1}, \Delta^{l}$ are hypoelliptic. We set $P=1-T^{l-1} Q^{l}$. Then $P$ is the orthogonal projection from $H^{l}$ onto $N\left(S^{l}\right)$.

We first prove that $P u \in A_{R}^{l}$ if $u \in A_{R}^{l}$. Since $u-P u \in N\left(S^{l}\right)^{\perp}=$ $R\left(T^{l-1}\right)=N\left(T^{l}\right)$ and since $A_{R}^{l} \subset D\left(T^{l}\right)$, we have $T^{l} P u=T^{l} u$ while $P u \in$ $N\left(S^{l}\right)$ implies $S^{l} P u=0$. To sum up

$$
T^{l} P u=T^{l} u, \quad S^{l} P u=0 .
$$

Hence the hypoellipticity of $\Delta^{l}$ implies that $P u$ is smooth. Set now $v=P u$ and note $\left\|f T^{l} v\right\|_{l+1}=\left\|f \partial^{l} u\right\|_{l+1}<+\infty$, if $f \in R$. Thus, to prove $v \in A_{R}^{l}$ we may only show that $\|f v\|_{l}<+\infty$ for any $f \in R$. If $f \in R_{0}=C$, then this is trivial. Suppose that $\|g v\|_{l}<+\infty$ if $g \in R_{k-1}$, and let $f \in R_{k}$. Let further $g_{j} j=1,2, \ldots, s_{f}$ satisfy (1.4.3) with $i=l-1$. Then

$$
\delta^{l} f v=\vartheta^{l-1}(\bar{f})^{*} v+f \delta^{l} v=\vartheta^{l-1}(\bar{f})^{*} v
$$

where we have used (1.4.7) in the last step. Thus

$$
\left\|\delta^{l}(f v)\right\|_{l-1} \leqq \sum_{j=1}^{s_{f}}\left\|g_{j} v\right\|_{l}
$$

The right hand side is finite by the inductive hypothesis. Since $\left\|\partial^{l} f v\right\|_{l+1}$ $=\left\|f T^{l} v\right\|_{l+1}<+\infty$ as remarked above, we conclude by Lemma 1.4.6 $\|f v\|_{l}<+\infty$, hence we have proved $v=P u \in A_{R}^{i}$ whenever $u \in A_{R}^{i}$. Obviously the map $A_{R}^{i} \ni u \rightarrow P u \in A_{R}^{i}$ is continuous.

From the characterization of $Q^{l}$ it follows

$$
T^{l-1} w=u-P u, S^{l-1} w=0
$$

where we have set $w=Q^{l} u$. If $u \in A_{R}^{l}$, then $u-P u \in A_{R}^{l}$, so, arguing 
just as we proved $P u \in A_{R}^{l}$ using (1.4.7), we obtain from (1.4.8) $w \in A_{R}^{l-1}$; the continuity of the map $A_{R}^{l} \rightarrow u \mapsto Q^{l} u=w \in A_{R}^{l-1}$ is also evident.

Q.E.D.

We conclude this section by studying briefly the relation between the estimation for $E^{\cdot}$ and that for the dual complex $F^{\bullet}$ : Let $F^{\cdot}$ be the dual of $E^{\cdot}$ as defined in $\S 1.3$ and $\langle\mid\rangle$ the duality homomorphism from $E^{-i} \otimes F^{i}$ to $\theta$. We define an anti-isomorphism between $E^{-i}$ and $F^{i}$ by setting

$$
\begin{gathered}
\left.<u,{ }^{*} v\right\rangle_{i} d v=\langle u \mid v\rangle \quad u \in E^{-i} \quad v \in F^{i} \\
* u=v \text { if and only if } u={ }^{*} v .
\end{gathered}
$$

We also introduce Hermitian structures $\langle,\rangle_{i}$ on $F^{i}$ as well as inner products $(,)_{i}$ and norms \|\|$_{i}$ for sections of $F^{i}, i \in \mathbf{Z}$ by

$$
\begin{gathered}
<v, v^{\prime}>_{i}=\left\langle * v, *^{*} v^{\prime}\right\rangle_{-i} \\
\left(v, v^{\prime}\right)_{i}=\int\left\langle v, v^{\prime}>_{i} d v \quad v, v^{\prime} \in C_{0}^{\infty}\left(F^{i}\right)\right. \\
\|v\|_{i}=(v, v)_{i} .
\end{gathered}
$$

We further write

$$
\left(\delta^{* i} u, v\right)_{i}=\left(u, \partial^{*(i-1)} v\right) \quad u \in C_{0}^{\circ}\left(F^{i}\right), v \in C_{0}^{\infty}\left(F^{i-1}\right)
$$

as a definition of the differential operator $\delta^{* i}: F^{i} \rightarrow F^{i-1}$. Then we have

Lemma 1.4.7 Under the above notation the following statements are valid.

(1) $\|* u\|_{i}=\|u\|_{-i}$.

(2) $\partial^{* i}(* u)=*\left(\delta^{-i} u\right)$.

(3) $\partial^{* i}(* u)=*\left(\partial^{-i} u\right)$.

(4) $f \in A\left(E^{\cdot}\right)$ if and only if $f \in A\left(F^{\cdot}\right)$.

(5) A subring $R$ of $A\left(E^{\cdot}\right)$ is well filtered if and only if $R$ is well filtered as a subring of $A\left(F^{*}\right)$. 
(6) The assumption $\left(\mathscr{A}_{q}\right)$ (resp. $\left(\mathscr{B}_{q}\right)$ ) is satisfied for $E^{\prime}$ if and only if $\left(\mathscr{B}_{-q}\right)$ (resp. $\left(\mathscr{A}_{-q}\right)$ ) is satisfied for $F$.

The proof is elementary and omitted.

\section{Application to Tangential Cauchy-Riemann Equation}

§2.1. Definitions. In this section we define the Dolbeault sequence for a closed real submanifold $M$ of a complex manifold $X$, and study some basic notions and facts concerning this sequence. (Note that the closedness assumption is inessential because any submanifold of a manifold has always a neighbourhood in which it is closed; we may only replace $X$ by such a neighbourhood if $M$ is not closed. The definition below, in fact, does not depend on the choice of such a neighbourhood.) Denote by $I^{0}$ the sheaf of germs of smooth functions in $X$ which vanish when restricted to $M$, and by $\Omega^{(p, q)}$ the sheaf of germs of smooth $(p, q)$ forms on $X$. Set further $\Omega=\sum \Omega^{(p, q)}$, then $\Omega$ is a sheaf of rings by the exterior multiplication. We denote by $I$ the sheaf of ideals of $\Omega$ generated by $I^{0}+\bar{\partial} I^{0}$ where $\bar{\partial}: \Omega^{(p, q)} \rightarrow \Omega^{(p, q+1)}$ is the differentiation of the usual Dolbeault sequence for $X$. Following Kohn and Rossi [9] we now define sheaves $D^{(p, q)}$ by defining $D=\sum D^{(p, q)}$ so that the sequence $0 \rightarrow I \rightarrow \Omega \rightarrow D$ $\rightarrow 0$ is exact. (Note that $I$ is homogeneous, that is, $I=\sum I^{(p, q)}, I^{(p, q)}=$ $I \cap \Omega^{(p, q)}$.) Since $\bar{\partial} I \subset I$, We can define a sheaf homomorphism $\bar{\partial}_{b}: D \rightarrow D$ such that $\bar{\partial}_{b} D^{(p, q)} \subset D^{(p, q+1)}$, making the following diagram commutative:

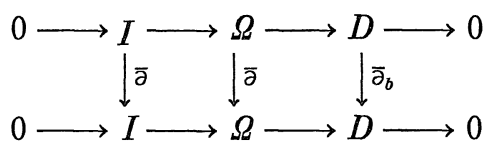

Thus we have obtained for $p \geqq 0$ the complex

$$
D^{(p, 0)} \stackrel{\bar{\partial}_{b}}{\longrightarrow} D^{(p, 1)} \stackrel{\bar{\partial}_{b}}{\longrightarrow} D^{(p, 2)} \stackrel{\bar{\partial}_{b}}{\longrightarrow} \cdots,
$$

which we shall call the p-th Dolbeault sequence for $M$. The equation $\bar{\partial}_{b} u=0$ for a section $u$ of $D^{0}$ is called usually the tangential CauchyRiemann equation of $M$. (For simplicity we write $D^{0}$ instead of $D^{(0,0)}$; 
note that $D^{0}$ is no other than the sheaf of germs of smooth functions over $M$.) We now assume that $M$ is generic, that is, $T_{z}(X)=T_{z}(M)+$ $\sqrt{-1} T_{z}(M)^{1)}$ (not necessarily the direct sum) for every $z \in M$. (Here $T(X), T(M)$ are the real tangent bundles of $X, M$ respectively, and $T(M)$ is regarded as a subspace of the complex vector space $T(X)$.) Then the sheaves $D^{(p, q)}$ are all locally free $D^{0}$-sheaf and $\bar{\partial}_{b}$ is a first order differential operator. Thus, for each $(p, q)$ there is a unique vector bundle such that $D^{(p, q)}$ is the sheaf of germs of sections of this vector bundle. To avoid making too many notations, we shall identify the sheaf $D^{(p, q)}$ with this vector bundle from now on, hence all $D^{(p, q)}$ should be regarded as vector bundles over $M$ in the sequel.

Now we shall give a more explicit description to bundles $D^{(p, q)}$. The exterior multiplication of $\Omega$ induces the multiplication of $D$ which satisfies the anti-commutativity relation with respect to the bidegree $(p, q)$. We thus obtain canonical bundle homomorphism $\Lambda^{p} D^{(1,0)} \otimes \Lambda^{q} D^{(0,1)} \rightarrow D^{(p, q)}$. This is actually an isomorphism as is easily seen. We shall investigate bundles $D^{(1.0)}, D^{(0,1)}$ more in detail. Denote by $D^{\circ}$ the subbundle of $T(M)$ whose fiber $D_{z}^{\circ}$ over each $Z \in M$ is $T_{z}(M) \cap \sqrt{-1} T_{z}(M)$, and by $T^{* c}(X)$ the complexified cotangent bundle of $X$ regarded as a real manifold. Then $T^{* c}(X)$ can be decomposed into a unique direct sum $T^{* c}(X)=T^{*(1,0)}(X)$ $+T^{*(0,1)}(X)$ so that $T^{*(1,0)}(X)$ (resp. $T^{*(0,1)}(X)$ ) denotes the subbundle of $\mathbb{C}$-linear (resp. C-antilinear) elements of $T^{* c}(X)$. (Note $T^{(0,1)}(X)=$ $\Omega^{(0,1)}, T^{*(1,0)}(X)=\Omega^{(0,1)}$.) Since $I^{(1,0)}=0, D^{(1,0)}=T^{(1,0)}(X) \mid M$. On the other hand, since $D^{\circ} \subset T(M) \subset T(X)$, there is a canonical duality homomorphism $D^{\circ} \underset{M}{\times} T^{*(0,1)}(X) \ni(x, y) \rightarrow\langle x| y>\in \mathbb{C}$ which is $\mathbb{C}$-linear in $y$ and $\mathrm{C}$-antilinear in $x$. (Note that $D^{\circ}$ is the largest complex subbundle of $T(X) \mid M$ contained in $T(M)$.) The bundle $D^{(0,1)}$ is a quotient bundle of $T^{*(0,1)}(X) \mid M$ and the kernel of projection $T^{(0,1)}(X) \mid M \rightarrow D^{(0,1)}$ is just the annihilator of $D^{\circ}$ in $T^{*(0,1)}(X) \mid M$. Thus we obtain also a canonical

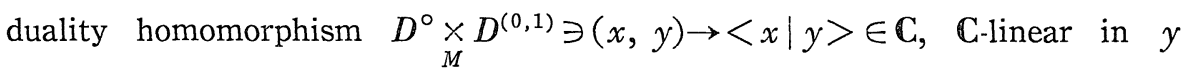
and $\mathrm{C}$-antilinear in $x$. Moreover $D_{z}^{\circ} \ni x=0$ (resp. $D_{z}^{(0,1)} \ni y=0$ ) if and only if $\langle x \mid y\rangle=0$ for all $y \in D^{(0,1)}$ (resp. for all $x \in D_{z}^{\circ}$ ). Thus, this

1) By $\sqrt{-1}$ we denote the real tensor field of $X$ defining the complex structure; never confuse this with the multiplication of $\sqrt{-1} \in \mathbb{C}$ in the complexification. 
duality homomorphism induces an anti-isomorphism between $D^{\circ *}$ and $D^{(0,1)}$. (We call a $\mathrm{C}$-antilinear bundle isomorphism an anti-isomorphism.) To sum up

Lemma 2.1.1. $D^{(1,0)}=T^{*(1,0)}(X) \mid M . \quad D^{(p, q)}$ is canonically isomorphic to $\Lambda^{p} D^{(1,0)} \otimes \Lambda^{q} D^{(0,1)}$, and $D^{(0,1)}$ is canonically anti-isomorphic to the dual $D^{\circ *}$ of $D^{\circ}$.

The last statement is very useful when introducing norms for the 0 -th Delbeault sequence $D^{(0, \cdot)} ;$ a Hermitian structure on the bundle $D^{\circ}$ induces automatically the Hermitian structures on $\Lambda^{q} D^{\circ}$, hence those on $D^{(0, q)}$.

Remark. fiber-dim $D_{\boldsymbol{C}} D^{\circ}=\operatorname{dim}_{\boldsymbol{C}} X-\operatorname{codim} \operatorname{Ri}_{\boldsymbol{R}} M$, fiber- $\operatorname{dim}_{\boldsymbol{C}} D^{(1,0)}=\operatorname{dim}_{\boldsymbol{C}} X$.

Finally we briefly describe a procedure for actually computing the Dolbeault sequences of the real submanifold $M$. Let $N=\operatorname{dim}_{C} X, n=$ $N-\operatorname{codim}_{\boldsymbol{R}} M$. Let further $\pi$ denote the canonical projection of $\Omega$ onto the quotient $D=\Omega / I$.

Definition 2.1.2. Let $\left(z_{1}, \ldots, z_{N}\right)$ be a system of coordinates in $X$. The real submanifold $M$ is said to be generically situated to the system $\left(z_{1}, \ldots, z_{N}\right)$ if and only if the sections $\pi\left(d z_{j}\right) j=1,2, \ldots, N ; \pi\left(d \bar{z}_{j}\right)$ $j=1,2, \ldots, n$ are linearly independent everywhere on $M$.

For simplicity we set $\zeta_{j}=\pi\left(d z_{j}\right), 1 \leqq j \leqq N ; \zeta_{\bar{k}}=\pi\left(d \overline{z_{k}}\right), 1 \leqq k \leqq n$, and more generally set

$$
\begin{aligned}
\zeta_{I, \bar{K}} & =\zeta_{i_{1}} \wedge \zeta_{i_{2}} \wedge \cdots \zeta_{i_{p}} \wedge \zeta_{\overline{k_{1}}} \cdots \wedge \zeta_{\overline{k_{p}}} \\
I & =\left\{i_{1}, i_{2}, \cdots i_{p}\right\}, K=\left\{k_{1}, \cdots, k_{p}\right\}
\end{aligned}
$$

where $I$ is a $p$-tuple of numbers between 1 and $N$, and $K$ is a $q$-tuple of numbers between 1 and $n$.

By Lemma 2.1.1 we can write a section $u$ of $D^{(p, q)}$ as a sum of the form $\sum_{|I|=p,|K|=q} u_{I, \bar{K}} \zeta_{I, \bar{K}}$ for some functions $u_{I, K}$ so that $u_{I, K}$ is an alternating function of $I$ and of $K$, that is, $u_{I, \bar{K}}$ changes sign if two indices in 
$I$ or $K$ are permuted. Thus we can identify $D^{(p, q)}$ with the trivial bundle $E^{(p, q)}$ of $\operatorname{rank}\left(\begin{array}{c}N \\ p\end{array}\right) \times\left(\begin{array}{c}n \\ q\end{array}\right)$ by identifying each $u$ with the alternating function $\left\{u_{I, K}\right\}$ of $I$ and $K$, which we can interpret as a section of $E^{(p, q)}$ of course.

Definition 2.1.3. The complex

$$
E^{(p, 0)} \stackrel{\bar{\partial}_{b}}{\longrightarrow} \cdots \stackrel{\bar{\partial}_{b}}{\longrightarrow} E^{(p, q)} \stackrel{\bar{\partial}_{b}}{\longrightarrow} E^{(p, q+1)} \stackrel{\bar{\partial}_{b}}{\longrightarrow} \ldots E^{(p, n)} \longrightarrow 0 \longrightarrow \cdots
$$

defined by the above identification is called the trivialization of the $p$-th Dolbeault sequence of $M$ by the coordinate system $\left(z, \ldots, z_{n}\right)$.

Now let us compute the differentiation $\bar{\partial}_{b}$ of this trivialization $E^{(p, \cdot)}$. We write for smooth function $u$

$$
\bar{\partial}_{b} u=\sum_{k=1}^{n} \bar{\partial} u_{k} \zeta_{\bar{k}}
$$

as a definition of the first order differential operator $\bar{\partial}_{k}, k=1,2, \ldots, n$. Note $\bar{\partial}_{b} \zeta_{I, \bar{K}}=0$ and note $\bar{\partial}_{b}(u \wedge v)=\bar{\partial}_{b} u \wedge v+(-1)^{\operatorname{deg} u} u \wedge \bar{\partial}_{b} v$. By computing $\bar{\partial}_{b}\left(\sum_{I, K} u_{I, \bar{K}} \zeta_{I, \bar{K}}\right)$ with these formulae in mind, we obtain for a section $u=\left\{u_{I, \bar{K}}\right\}$ of $E^{(p, q)}$

$$
\begin{array}{r}
\left(\bar{\partial}^{b} u\right)_{I, \bar{L}}=(-1)^{p} \sum_{j=1}^{q+1}(-1)^{j-1} \bar{\partial}_{l_{j}} u_{I, \bar{L} \backslash l_{j}} \\
L=\left\{l_{1}, \ldots l_{2}, \ldots, l_{q+1}\right\}
\end{array}
$$

where $L \backslash l_{j}$ denote the $q$-tuple of the remaining indices after deleting $l_{j}$ from $L$. In (2.2.1) the index $I$ does not play no essential role evidently. Thus the $p$-th Dolbeault sequence $D^{(p, \cdot)}$ is isomorphic to $\left(\begin{array}{l}N \\ p\end{array}\right)$-fold product of the 0 -th Dolbeault sequence $D^{(0, \cdot)}$. (This isomorphism is not canonical; it depends on the choice of the coordinates $\left(z_{1}, \ldots, z_{N}\right)$.) Since locally there always exists a coordinate system for which a given real submanifold is generically situated, it suffices to study the 0 -th Dolbeault sequence only, at least as far as local problems are in question. 
§ 2.2. Basic estimates. Let $V$ be an $n$-dimensional complex vector space with dual $V^{*}$. Choose basis $\left\{e_{1}, \ldots, e_{n}\right\}$ in $V$ and $\left\{e_{1}^{*}, \ldots, e_{n}^{*}\right\}$ in $V^{*}$ so that $\left\langle e_{i} \mid e_{j}^{*}\right\rangle=\delta_{i j}$. Here $\delta_{i j}$ denotes the Kronecker delta. Given a Hermitian form $h(y, z), y, z \in V$ on $V$, we define a differential operator $C_{0}^{\infty}(V) \stackrel{\bar{\partial}_{(h)}}{\longrightarrow} C_{0}^{\infty}\left(V, V^{*}\right)$ by setting

$$
\bar{\partial}_{(h)} u=\sum_{j=1}^{n}\left(\frac{\partial u}{\partial \bar{z}_{j}}-\frac{\partial h(z, z)}{\partial \bar{z}_{j}} u\right) e_{j}^{*}
$$

where we have defined the coordinate system $\left(z, \ldots, z_{n}\right)$ so that this expresses the element $z_{1} e_{1}+\cdots+z_{n} e_{n}$ in $V$. Actually the operator $\bar{\partial}_{(h)}$ does not depend on the choice of $\left\{e_{1}, \ldots, e_{n}\right\}$; it depends only on $h$. We get also differential operators

$$
C_{0}^{\infty}\left(V, \Lambda^{q} V^{*}\right) \stackrel{\bar{\partial}_{(h)}}{\longrightarrow} C_{0}^{\infty}\left(V, \Lambda^{q+1} V^{*}\right)
$$

by requiring that

$$
\bar{\partial}_{(h)}(u w)=\bar{\partial}_{(h)} u \wedge w \quad u \in C_{0}^{\infty}(V), w \in \Lambda^{q} V^{*} .
$$

Introducing a Hermitian inner product $\langle,>$ and norm $|$ on $V$, we let now $V$ be an $n$-dimensional Hilbert space. Given a Hermitian form $h(y, z)$ on $V$, we can find a unique Hermitian endomorphism $\tilde{h}$ of $V$ such that $h(y, z)=\langle\tilde{h} y, z\rangle(=\langle y, \tilde{h} z\rangle)$ and we will define the eigenvalues of $h$ as the characteristic values of $\tilde{h}$. As usual we shall now extend the inner product $\left\langle,>\right.$ over $\Lambda^{q} V^{*}, q=1,2, \ldots, n$. First note that there is a unique $\mathbb{C}$-antilinear isomorphism $V \in z \rightarrow \tilde{z} \in V^{*}$ such that $\langle y, z\rangle=\langle y \mid \tilde{z}\rangle$. We introduce on $V^{*}$ the Hermitian structure $<,>$ setting $\langle\tilde{z}, \tilde{y}\rangle=\langle y, z\rangle$ and define $<,>$ on $\Lambda^{q} V^{*}$ so that $\left\langle y^{1} \wedge y^{2} \wedge\right.$ $\left.\ldots \wedge y^{q}, z^{1} \wedge z^{2} \wedge \ldots \wedge z^{q}\right\rangle=\operatorname{det}\left|\left\langle y^{j}, z^{k}\right\rangle\right|$ if $y^{1}, \ldots, y^{q} ; z^{1}, \ldots, z^{q} \in V^{*}$. Now take an element $v$ of $\Lambda^{n} V^{*}$ such that $|v|=1$. We regard $v$ as a holomorphic $n$-form on $V$, so that $\sqrt{-1}^{n} v \wedge \bar{v}$ is a volume element (measure) of $V$. Set $d G_{t}(V)=\sqrt{-1}^{n} e^{-t|z|^{2}} v \wedge \bar{v}$. We can then form the adjoint $\delta_{(h)}^{t}$ of $\bar{\partial}_{(h)}$ by setting 


$$
\begin{aligned}
\int\left\langle\bar{\partial}_{(h)} u, v>d G_{t}(V)\right. & =\int\left\langle u, \delta_{(h)}^{t} v>d G_{t}(V)\right. \\
u & \in C_{0}^{\infty}\left(V, \Lambda^{q-1} V^{*}\right), v \in C_{0}^{\infty}\left(V, \Lambda^{q} V^{*}\right) .
\end{aligned}
$$

Now let $\lambda_{1} \leqq \lambda_{2} \leqq \cdots \leqq \lambda_{n}$ be the eigenvalues of a Hermitian form $h$. For any $q$-tuple $K$ of different indices between 1 and $n$ we set

$$
c_{K}(h)=\max \left\{\sum_{j \in K} a_{j} \mid \sum_{j \in K} \lambda_{j} a_{j}=0 ; 0 \leqq a_{j} \leqq 1, j \in K\right\} .
$$

Further, we set for $1 \leqq q \leqq n$

$$
c_{q}(h)=\min _{|K|=q} c_{K}(h)
$$

where the minimum should be taken over the set of all $q$-tuples $K$ of different indices between 1 and $n$. Then our main result here is the following

Theorem 2.2.1. Let the notation be as above. Then the following estimates are valid:

$$
\begin{gathered}
c_{q}(h) t \int|u|^{2} d G_{t}(V) \leqq \int\left|\bar{\partial}_{(h)} u\right|^{2} d G_{t}(V)+\int\left|\delta_{(h)}^{t} u\right|^{2} d G_{t}(V) . \\
-c_{n-q}(h) t \int|u|^{2} d G_{t}(V) \leqq \int\left|\bar{\partial}_{(h)} u\right|^{2} d G_{t}\left((V)+\int\left|\delta_{(h)}^{t} u\right|^{2} d G_{t}(V) .\right. \\
u \in C_{0}^{\infty}\left(V, \Lambda^{q} V^{*}\right) .
\end{gathered}
$$

Before proving this theorem we shall write $\delta_{(h)}^{t}$ in a more explicit form: We first assume that the base $\left\{e_{1}, \ldots, e_{n}\right\}$ is orthonormal and that $h$ is diagonalized for $\left\{e_{1}, \ldots, e_{n}\right\}$, that is, $\partial h(z, z) / \partial \bar{z}_{j}=\lambda_{j} z_{j}, 1 \leqq$ $j \leqq n$. (This assumption leads to no loss of generality since $c_{p}(h)=c_{p}\left(h^{\prime}\right)$ whenever $h$ and $h^{\prime}$ are unitarily equivalent and since the measures $d G_{t}(V)$ have also the unitary invariance.) We set

$$
\begin{array}{ll}
\bar{\partial}_{j}=\frac{\partial}{\partial \bar{z}_{j}}-\lambda_{j} z_{j} & 1 \leqq j \leqq n \\
\delta_{j}^{t}=\frac{\partial}{\partial z_{j}}+\left(\lambda_{j}-t\right) \overline{z_{j}} & 1 \leqq j \leqq n .
\end{array}
$$


By integration by parts we have

$$
\left(\bar{\partial}_{j} u, v\right)_{t}+\left(u, \delta_{j}^{t} v\right)_{t}=0 \quad u, v \in C_{0}^{\infty}(V)
$$

where we have set $(u, v)_{t}=\int u \bar{v} d G_{t}(V)$. We set for any $q$-tuple $K=$ $\left\{k_{1}, \ldots, k_{q}\right\}$ of indices from $\{1,2, \ldots, n\}$

$$
e_{K}^{*}=e_{k_{1}}^{*} \wedge \cdots \wedge e_{k_{q}}^{*} \text {. }
$$

We can write then

$$
u=\sum_{|K|=q} u_{K} e_{K}^{*} \quad u \in C_{0}^{\infty}\left(V, \Lambda^{q} V^{*}\right)
$$

so that $u_{K}$ is alternating in $K$, and we identify $u$ and $\left\{u_{K}\right\}$. Then, by the rule (2.2.1) we obtain

$$
\begin{aligned}
\left(\bar{\partial}_{(h)} u\right)_{L}=\sum_{j=1}^{q+1}(-1)^{j-1} \partial_{l_{j}} u_{L \backslash l_{j}} & \\
& u \in C_{0}^{\infty}\left(V, \Lambda^{q} V^{*}\right), L=\left\{l_{1}, \ldots, l_{q+1}\right\} .
\end{aligned}
$$

From (2.2.4), (2.2.5) it follows now

$$
\left(\delta_{(h)}^{t} u\right)_{J}=-\sum \delta_{j}^{t} u_{j J} \quad|J|=q-1
$$

where $j J$ denotes the $q$-tuple of indices obtained by adding $j$ to $J$ as a first index.

Lemma 2.2.2. The notation being as above, it holds for $u=\left\{u_{K}\right\} \in$ $C_{0}^{\infty}\left(V, \Lambda^{q} V^{*}\right)$

$$
\begin{aligned}
& \int\left(\left|\bar{\partial}_{(h)} u\right|^{2}+\left|\delta_{(h)}^{t} u\right|^{2}\right) d G_{t}(V) \\
& =\sum_{|K|=q}\left(\sum_{j \in K^{c}}\left\|\bar{\partial}_{j} u_{K}\right\|_{t}^{2}+\sum_{j \in K}\left\|\delta_{j}^{t} u_{K}\right\|_{t}^{2}\right)
\end{aligned}
$$

where $K^{c}$ denotes the complement $\{1,2, \ldots,\} \backslash K$ and $\|u\|_{t}^{2}=(u, u)_{t}$ for $u \in C_{0}^{\infty}(V)$.

Proof. As proved in Hörmander [4] we have 


$$
\begin{aligned}
\left|\bar{\partial}_{(h)} u\right|^{2} & =\sum_{|K|=q} \sum_{j=1}^{n}\left|\bar{\partial}_{j} u_{K}\right|^{3}-\sum_{|J|=q-1} \sum_{j, k=1}^{n} \bar{\partial}_{k} u_{j J} \overline{\bar{\partial}_{j} u_{K J}} \\
& =\sum_{|K|=q} \sum_{j \in K^{c}}\left|\bar{\partial}_{j} u_{K}\right|^{2}-\sum_{|J|=q-1} \sum_{j \neq k} \bar{\partial}_{k} u_{j J} \overline{\bar{\partial}_{j} u_{k J}}
\end{aligned}
$$

Also we have

$$
\begin{aligned}
\left|\delta_{(h)}^{t} u\right|^{2} & =\sum_{|J|=q-1} \sum_{j, k} \delta_{j}^{t} u_{j J} \overline{\delta_{k}^{t} u_{k J}} \\
& =\sum_{|K|=q} \sum_{j \in K}\left|\delta_{j}^{t} u_{K}\right|^{2}+\sum_{|J|=q-1} \sum_{j \neq k} \delta_{j}^{t} u_{j J} \overline{\delta_{k}^{t} u_{k J}}
\end{aligned}
$$

To sum up we obtain

$$
\begin{aligned}
\left|\bar{\partial}_{(h)} u\right|^{2}+\left|\delta_{(h)}^{t} u\right|^{2}= & \sum_{|K|=q}\left(\sum_{j \in K}\left|\delta_{j}^{t} u_{K}\right|^{2}+\sum_{j \in K^{c}}\left|\bar{\partial}_{j} u_{K}\right|^{2}\right) \\
& +\sum_{|J|=q-1} \sum_{j \neq k}\left(\delta_{j}^{t} u_{j J} \overline{\delta_{k}^{t} u_{k J}}-\bar{\partial}_{k} u_{j J} \overline{\bar{\partial}_{j} u_{k J}}\right)
\end{aligned}
$$

which, combined with (2.2.4), proves

$$
\begin{aligned}
\int\left(\left|\bar{\partial}_{(h)} u\right|^{2}+\left|\delta_{(h)}^{t} u\right|^{2}\right) d G_{t}(V) & \\
= & \sum_{|K|=q}\left(\sum_{j \in K}\left\|\delta_{j}^{t} u_{K}\right\|_{t}^{2}+\sum_{j \in K^{c}}\left\|\bar{\partial}_{j} u_{K}\right\|_{t}^{2}\right) \\
& \quad+\sum_{|J|=q} \sum_{j \neq k}\left(\left[\delta_{j}^{t}, \bar{\partial}_{k}\right] u_{j J}, u_{k J}\right)_{t} .
\end{aligned}
$$

The second term here actually vanishes since $\left[\delta_{j}^{t}, \bar{\partial}_{k}\right]=0$ whenever $j \neq k$. Thus the proof is complete.

Lemma 2.2.3. Let the notation be as above. Then

$$
\begin{aligned}
& \sum_{j \in K}\left\|\delta_{j}^{t} u\right\|_{t}^{2} \geqq t c_{K}(h)\|u\|_{t}^{2} \\
& \sum_{j \in K}\left\|\bar{\partial}_{j} u\right\|_{t}^{2} \geqq-t c_{K}(h)\|u\|_{t}^{2}
\end{aligned}
$$

for $u \in C_{0}^{\infty}(V)$ and for every $q$-tuple $K$ of different indices between 1 and $n$.

Proof. Note $\left[\delta_{t}^{j}, \bar{\partial}_{j}\right]=t-2 \lambda_{j}$. Hence 


$$
\left\|\delta_{j}^{t} u\right\|_{t}^{2}-\left\|\bar{\partial}_{j} u\right\|_{t}^{2}=\left(t-2 \lambda_{j}\right)\|u\|_{t}^{2} .
$$

Suppose now $\sum_{j \in K} a_{j} \lambda_{j}=0 ; 0 \leqq a_{j} \leqq 1, j \in K$. Then

$$
\begin{aligned}
\sum_{j \in K}\left\|\delta_{j}^{t} u\right\|_{t}^{2} & \geqq \sum_{j \in K} a_{j}\left\|\delta_{j}^{t} u\right\|_{t}^{2} \\
& =\sum_{j \in K} a_{j}\left\|\bar{\partial}_{j} u\right\|_{t}^{2}+t\left(\sum_{j \in K} a_{j}\right)\|u\|_{t}^{2} \\
& \geqq t\left(\sum_{j \in K} a_{j}\right)\|u\|_{t}^{2}, \\
\sum_{j \in K}\left\|\bar{\partial}^{j} u\right\|_{t}^{2} & \geqq \sum_{j \in K} a_{j}\left\|\bar{\partial}^{j} u\right\|_{t}^{2} \\
& =\sum_{j \in K} a_{j}\left\|\delta_{j}^{t} u\right\|_{t}^{2}-t\left(\sum_{j \in K} a_{j}\right)\|u\|_{t}^{2} \\
& \geqq-t\left(\sum_{j \in K} a_{j}\right)\|u\|_{t}^{2} .
\end{aligned}
$$

$\begin{array}{ll}\text { From these and from (2.2.2) we obtain (2.2.7), (2.2.7)'. } & \text { Q.E.D. }\end{array}$

Proof of Theorem 2.2.1. Obvious by Lemma 2.2.2 and Lemma 2.2.3.

Note that nothing follows from Theorem 2.2.1 when $t=0$, so we mention now another type of estimates concerning the norm $\left(\int|u|^{2} d G_{0}(V)\right)^{\frac{1}{2}}$; these are due to Hörmander [6]. Let $\lambda_{1} \leqq \lambda_{2} \leqq \cdots \leqq \lambda_{n}$ be the eigenvalues of a Hermitian form $h$ and set $\lambda_{j}^{+}=\max \left(0, \lambda_{j}\right), \lambda_{j}^{-}=\max \left(0,-\lambda_{j}\right)$. We define the function $c_{q}^{*}$ by

$$
c_{q}^{*}(h)=\min _{|K|=q}\left(\sum_{j \in K} \lambda_{j}^{-}+\sum_{j \in K^{c}} \lambda_{j}^{+}\right)
$$

where the minimum should be taken over the set of all $q$-tuples $K$ of different indices between 1 and $n$.

Theorem 2.2.4 ([6]). The notation being as above,

$$
\begin{aligned}
& c_{q}^{*}(h) \int|u|^{2} d G_{0}(V) \\
& \leqq \int\left|\bar{\partial}_{(h)} u\right|^{2} d G_{0}(V)+\int\left|\delta_{(h)}^{0} u\right|^{2} d G_{0}(V)
\end{aligned}
$$


if $u \in C_{0}^{\infty}\left(V, \Lambda^{q} V^{*}\right)$.

This can be proved by Lemma 2.2 .2 combined with the estimates

$$
\left\|\bar{\partial}_{j} u\right\|_{2}^{0} \geqq \lambda_{j}^{+}\|u\|_{0}^{2},\left\|\delta_{j} u\right\|_{0} \geqq \lambda^{-}\|u\|_{0}^{2} \quad u \in C_{0}^{\infty}(V)
$$

which are also consequences from (2.2.4).

Finally we shall list up some elementary properties of the functions $c_{q}, c_{q}^{*}$ without proof. Let $H(V)$ be the set of hermitian forms on $V$ and set $H_{q}(V)=\left\{h \in H(V) \mid c_{q}(h)>0\right\}, H_{q}^{*}(V)=\left\{h \in H(V) \mid c_{q}^{*}(h)>0\right\}$. Further, let $H_{q}(V)^{\circ}, H_{q}^{*}(V)^{\circ}$ be the interiors of $H_{q}(V), H_{q}^{*}(V)$ respectively.

Lemma 2.2.5. Let $\lambda_{1} \leqq \lambda_{2} \leqq \cdots \leqq \lambda_{n}$ be the eigenvalues of a Hermitian form $h$. Then

(1) $h \in H_{q}(V)$ if and only if $\lambda_{q} \geqq 0$ and $\lambda_{n-q+1} \leqq 0$

(2) $h \in H_{q}^{*}(V)$ if and only if $\lambda_{q}>0$ or $\lambda_{n-q+1}<0$

(3) $h \in H_{q}(V)^{\circ}$ if and only if $\lambda_{q}>0$ and $\lambda_{n-q+1}<0$

(4) $H_{q}^{*}(V)^{\circ}=H_{q}^{*}(V)$, i.e. $H_{q}^{*}(V)$ is open in $H(V)$

(5) $c_{q}(h)=c_{q}\left(h^{\prime}\right), c_{q}^{*}(h)=c_{q}^{*}\left(h^{\prime}\right)$ whenever $h, h^{\prime}$ are unitarily equivalent.

(6) $c_{q}(s h)=c_{q}(h)$ for any real $s \neq 0, c_{q}(0)=q$.

(7) $c_{q}^{*}(s h)=s c_{q}^{*}(h)$ for $s>0$

(8) $c_{q}, c_{q}^{*}$ are lower semi-continuous on $H_{q}(V)^{\circ}, H_{q}^{*}(V)$ respectively.

$\S 2.3$. Standard real submanifolds. In this section we shall show that Theorem 2.2.1 implies certain estimates concerning some Dirichlet norms defined for the Dolbeault sequences of a remarkable class of real submanifolds, namely, the standard real submanifolds of the second kind in the sence of Tanaka [18].

Definition 2.3.1. Let $V, W$ be vector spaces over $\mathbf{C}, \mathbf{R}$ respectively, and let $W^{c}$ be the complexification of $W$. A map $f: V \times V \rightarrow W^{c}$ is called a $W$-hermitian form on $V$ if and only if (1) $f(y, z)$ is C-linear in $y, \quad(2) \quad f(y, z)=\overline{f(z, y)}(\overline{s+i t}=s-i t$ if $s, t \in W)$, (3) the elements $f(y, z)+f(z, y) y, z \in V$ span $W$. The standard real submanifold associated with a $W$-hermitian form $f$ on $V$ is the real submanifold 


$$
M_{f}=\left\{\left(z, x+\sqrt{-1} f(z, z) \in V \times W^{c} \mid(z, x) \in V \times W\right\}\right.
$$

situated in complex vector space $V \times W^{c}$.

Remark. By standard real submanifolds Tanaka means much wider class of real submanifolds than those defind above. All of the $M_{f}$ corresponds to those of the second kind in the sense of [18].

As already studied in [18], $M_{f}$ has some good properties, for example, $M_{f}$ is a Lie group in the following way. Let $p$ be the projection of $V \times W^{c}$ onto $V$. Set for $\xi, \xi^{\prime} \in V \times W^{c}$

$$
\rho_{\xi}\left(\xi^{\prime}\right)=\xi+\xi^{\prime}+2 \sqrt{-1} f\left(p\left(\xi^{\prime}\right), p(\xi)\right) .
$$

Then the map $\rho_{\xi}$ is a complex affine transformation of $V \times W^{c}$ into itself; moreover, if $\xi \in M_{f}$, then $\rho_{\xi}\left(M_{f}\right)=M_{f}$. $M_{f}$ becomes a Lie group if one takes $\rho_{\xi}\left(\xi^{\prime}\right)$ as the product $\xi, \xi^{\prime}$ of elements $\xi, \xi^{\prime} \in M_{f}$.

Since the left multiplications of $M_{f}$ are extended to affine transformations of $V \times W^{c}$, they induce bundle automorphisms of $T\left(V \times W^{c}\right) \mid M_{f}$. Of course each of these automorphisms maps the subbundles $T\left(M_{f}\right), \sqrt{-1}$ $T\left(M_{f}\right)$ onto themselves, in particular $D_{f}^{\circ}=T\left(M_{f}\right) \cap \sqrt{-1} T\left(M_{f}\right), T\left(M_{f}\right)+$ $\sqrt{-1} T\left(M_{f}\right)$ are subbundles of $T\left(V \times W^{c}\right) \mid M_{f}$. But $T\left(M_{f}\right)+\sqrt{-1} T\left(M_{f}\right)$ $=T\left(V \times W^{c}\right) \mid M_{f} ;$ for note $V \times W^{c}=(V \times W)+\sqrt{-1}(V \times W)$, and $T_{0}\left(M_{f}\right)$ $=V \times W$ when $V \times W^{c}$ identified with its tangent space at the origin. Thus $M_{f}$ is generic. Since the Dolbeault sequences for $M_{f}$

$$
\begin{gathered}
D_{f}^{(p, 0)} \stackrel{\bar{\partial}_{f}}{\longrightarrow} D_{f}^{(p, 1)} \stackrel{\bar{\partial}_{f}}{\longrightarrow} D_{f}^{(p, 2)} \stackrel{\bar{\partial}_{f}}{\longrightarrow} \cdots \stackrel{\bar{\partial}_{f}}{\longrightarrow} D_{f}^{(p, n)} \longrightarrow 0 \\
0 \leqq p, n=\operatorname{dim} \mathbf{C}^{V}
\end{gathered}
$$

are also invariant under holomorphic transformations of $M_{f}$, the left multiplications of $M_{f}$ induce automorphisms of these sequences. Hence the bundles $D_{f}^{\circ}$ and $D_{f}^{(0, q)}(q \geqq 0)$ can be naturally indentified with the products of $M_{f}$ and of their fibers over one point of $M_{f}$, e.g. over the origin. Thus, in particular $D_{f}^{\circ}$ is canonically isomorphic to the trivial bundle $M_{f} \times V \quad(V=(V \times W) \cap \sqrt{-1}(V \times W))$, and $D_{f}^{(0, q)}$ are anti-isomorphic to $M_{f} \times \Lambda^{q} V^{*}$ (see Lemma 2.1.1). 
Introduce now a Hermitian inner product $<,>$ on $V$. Then this defines canonically Hermitian structures $\langle$,$\rangle on the vector bundles$ $D_{f}^{(0, q)} q=0,1,2, \cdots n$, according to the above identification by the group structure of $M_{f}$. By the diffeomorphism $V \times W \ni(z, x) \rightarrow(z, x+\sqrt{-1} f$ $(z, z)) \in M_{f}$, one can induce a measure $d V_{t}$ on $M_{f}$ from measure $d G_{t}(V)$ $d x^{2)}$ on $V \times W$, where we have chosen a translation invariant measure $d x$ on $W$. Further, we define the adjoint $\delta_{f}^{t}$ of $\bar{\partial}_{f}$ by setting

$$
\begin{aligned}
\int\left\langle u, \delta_{f}^{t} v>d V_{t}=\int\left\langle\bar{\partial}_{f} u,\right.\right. & v>d V_{t} \\
u & \in C_{0}^{\infty}\left(D^{(0, q-1)}\right), v \in C_{0}^{\infty}\left(D^{(0, q)}\right) .
\end{aligned}
$$

Now set for $\xi \in W^{*}$

$$
h(\xi)(y, z)=\langle\xi| f(y, z)>\quad y, z \in V
$$

to define the Hermitian form $h(\xi)$ on $V$. Let $c_{p}$ be the function defined by $(2.2 .2)^{\prime}$ as in the previous section with respect to the inner product $<$, $>$ on $V$, and set

$$
c_{p}(f)=\min _{\xi \in W^{*}} c_{p}(h(\xi))
$$

Then we obtain as the main result of this section

\section{Theorem 2.3.2. The notation being as above}

$$
\begin{gathered}
t c_{q}(f) \int|u|^{2} d V_{t} \leqq \int\left(\left|\bar{\partial}_{f} u\right|^{2}+\left|\delta_{f}^{t} u\right|^{2}\right) d V_{t} \\
u \in C_{0}^{\infty}\left(D_{f}^{(0, q)}\right) \\
-t c_{n-q}(f) \int|u|^{2} d V_{t} \leqq \int\left(\left|\bar{\partial}_{f} u\right|^{2}+\left|\delta_{f}^{t} u\right|^{2}\right) d V_{t} .
\end{gathered}
$$

Proof. (i) Proof of (2.3.2). We identify $V \times W$ with $M_{f}$ by the $\operatorname{map}(z, x) \mapsto(z, x+\sqrt{-1} f(x, x))$, and sections of $D_{f}^{(0, q)}$ with $\Lambda^{q} V^{*}$-valued functions over $V \times W$ (in view of the anti-isomorphism between $D_{f}^{(0, q)}$

2) For the notation $d G_{t}(V)$ see $\$ 2.2$. 
and the trivial bundle $M_{f} \times \Lambda^{q} V^{*}$ over $M_{f}$ ). Then the Dolbeault sequence $D_{f}^{(0, \cdot)}$ is regarded as a complex of the following form:

$$
\cdots \stackrel{\bar{\partial}_{f}}{\longrightarrow} C_{0}^{\infty}\left(V \times W, \Lambda^{q} V^{*}\right) \stackrel{\bar{\partial}_{f}}{\longrightarrow} C_{0}^{\infty}\left(V \times W, \Lambda^{q+1} V^{*}\right) \longrightarrow \cdots
$$

and it suffices to prove

$$
\begin{aligned}
c_{q}(f) \int|u|^{2} d G_{t}(V) d x \leqq \int\left(\left|\bar{\partial}_{f} u\right|^{2}+\left|\delta_{f}^{t} u\right|^{2}\right) d G_{t}(V) d x & \\
& \quad u \in C_{0}^{\infty}\left(V \times W, \Lambda^{q} V^{*}\right) .
\end{aligned}
$$

To show this we introduce the partial Fourier transform:

$$
\tilde{u}(z, \xi)=\int e^{-i<x|\xi\rangle} u(z, x) d x \quad(z, \xi) \in V \times W^{*} .
$$

Then a simple computation shows

$$
\begin{aligned}
& \widetilde{\bar{\partial}_{f} u}(z, \xi)=\bar{\partial}_{(h(\xi))} \tilde{u}(z, \xi) \\
& \widetilde{\delta_{f}^{t} u}(z, \xi)=\delta_{(h(\xi))}^{t} \tilde{u}(z, \xi)
\end{aligned}
$$

where we have let the notation $\bar{\partial}_{(h)}, \delta_{(h)}^{t}$ be as in $\S 2.1$. Hence we obtain by applying Theorem 2.2.1

$$
\begin{aligned}
& t c_{q}(f) \int|\tilde{u}(z, \xi)|^{2} d G_{t}(V) \leqq t c_{q}(h(\xi)) \int|\tilde{u}(z, \xi)|^{2} d G_{t}(V) \\
& \leqq \int\left(\left|\widetilde{\bar{\partial}_{f} u}(z, \xi)\right|^{2}+\mid \widetilde{\delta_{f}^{t} u}\left(\left.(z, \xi)\right|^{2}\right) d G_{t}(V) \quad u \in C_{0}^{\infty}\left(V \times W, \Lambda^{q} V^{*}\right) .\right.
\end{aligned}
$$

Choose a translation invariant measure $d \xi$ so that $\langle d \xi \mid d x\rangle=\pi^{-d}$ ( $d=$ $\operatorname{dim}_{\boldsymbol{R}} W$ ). (We regard here $d x, d \xi$ as elements of $\Lambda^{d} W, \Lambda^{d} W^{*}$ respectively.) Then the Plancherel formula $\int|u(z, x)|^{2} d x=\int|\tilde{u}(z, \xi)|^{2} d \xi$ proves (2.3.2).

(ii) Proof of $(2.3 .2)^{\prime}$. Similarly proved.

We shall now give a sufficient condition for $c_{q}(f)$ to be positive.

Definition 2.3.3. A $W$-hermitian form $f$ on $V$ is said to satisfy 
the condition $\nu_{q}$ if and only if for every $0 \neq \xi \in W^{*}$ one can find a subspace of $V$ of dimension at least $q+1$ on which the Hermitian form $h(\xi)(y, z)=\langle\xi| f(y, z)>y, z \in V$ is positive definite.

Lemma 2.3.4. If $f$ satisfies the condition $\nu_{q_{0}}$, then $c_{q}(f)>0$ for $n-q_{0} \leqq q \leqq n$.

Proof. Since the condition $\nu_{q_{0}}$ implies the condition $\nu_{p}$ if $0 \leqq p \leqq q_{0}$, it suffices to prove $c_{n-q_{0}}(f)>0$. By (3) of Lemma 2.2.5, $h(\xi) \in H_{n-q_{0}}(V)^{\circ}$ if $\xi \neq 0, \xi \in W^{*}$. Then the compactness of the projective space $\left(W^{*} \backslash 0\right) /$ $(R \backslash 0)$, together with (6) and (8) of Lemma 2.2.5, implies

$$
\min _{\xi \in W^{*} \backslash 0} c_{n-q_{0}}(h(\xi))>0 .
$$

Since $c_{n-q_{0}}(0)=n-q_{0}, \quad c_{n-q_{0}}(f)>0$ is proved.

Q.E.D.

We conclude this section by indicating the identity of the trivialization for the complex $D^{(0, \cdot)}$ in the sence of Definition 2.1.3 with that done here by using the group structure of $M_{f}$. Let $\left\{e_{1}, \ldots, e_{n}\right\}$ be an orthonormal base in $V$ and choose a base $\left\{e_{1}{ }^{\prime}, \ldots, e_{d}{ }^{\prime}\right\}$ in the real vector space $W$. We set for $z \in V \times W^{c}$

$$
z=\sum_{i=1}^{n} z_{i} e_{i}+\sum_{i=1}^{d} z_{n+i} e_{i}^{\prime}
$$

to define the coordinate system $\left(z_{1}, z_{2}, \ldots, z_{N}\right), N=n+d$ in $V \times W^{c}$. Then $M_{f}$ is obviously generically situated in the sense of Definition 2.1.2. As remarked at the end of $\S 2.1$, the sections $\zeta_{\bar{k}}=\bar{\partial}_{b} \bar{z}_{k}, 1 \leqq k \leqq n$ form a frame in $D_{f}^{(0,1)}$ and the sections $\zeta_{\bar{K}}=\zeta_{\bar{k}_{1}} \wedge \cdots \wedge \zeta_{\bar{k}_{q}}$, where $K=\left\{k_{1}, \ldots, k_{q}\right\}$ runs over all $q$-tuple from $\{1,2, \ldots, n\}$, form a frame in $D_{f}^{(0, q)}$. It is obvious that $\rho_{\zeta} *\left(d \bar{z}_{k}\right)=d \bar{z}_{k}, 1 \leqq k \leqq n$ where $\rho_{\zeta}$ was defined by (2.3.1); therefore the sections $\zeta_{\bar{k}}, 1 \leqq k \leqq n$ are invariant under the left multiplication of $M_{f}$ hence the sections $\zeta_{\bar{K}},|K|=q 1 \leqq q \leqq n$ are also invariant. It is obvious that $\langle u, v\rangle$, defined by the anti-isomorphism between the trivial bundle $M_{f} \times \Lambda^{q} V^{*}$ and $D_{f}^{(0, q)}$, coincides with $\sum_{|K|=q} u_{\bar{K}} \overline{v_{\bar{K}}}$ if $u, v \in$ $C_{0}^{\infty}\left(D_{f}^{(0, q)}\right)$. Here, of course we have set $u=\sum_{|K|=q} u_{\bar{K}} \zeta_{\bar{K}}$ so that $u_{\bar{K}}$ is 
alternating in $K$.

§2.4. Real submanifolds of the second kind. In this section we shall introduce some important geometrical regularity conditions for real submanifolds, and prove a subellipticity theorem for the Dolbeault sequences, referring to a result of Hörmander [6].

Let $M$ be a generic submanifold of a complex manifold $X$ and let $N=\operatorname{dim}_{\boldsymbol{C}} X, n=N-\operatorname{codim}_{\boldsymbol{R}} M . \quad$ Define as in $\S 2.1$ the subbundle $D^{\circ}$ of $T(M)$ as the intersection $T(M) \cap \sqrt{-1} T(M)$.

Defininition 2.4.1. The real submanifold $M$ is said to be of the second kind if and only if every real vector field can be written as a sum of sections of $D^{\circ}$ and of brackets of sections of $D^{\circ}$, that is, $T(M)=$ $D^{\circ}+\left[D^{\circ}, D^{\circ}\right]$.

Let $\Xi$ denote the quotient bundle $T(M) / D^{\circ}$. If $M$ is of the second kind, we can define for each $z \in M$, a surjective anti-symmetric bilinear map $D_{z}^{\circ} \otimes D_{z}^{\circ} \ni(x \otimes y) \rightarrow[x, y]^{z} \in \Xi_{z}$ so that $\left[X_{z}, Y_{z}\right]^{z}$ is the residue class of the value $[X, Y]_{z} \in T_{z}(M)$ if $X, Y$ are sections of $D^{\circ}$. From the vanishing of the Nijenhuis tensor it follows immediately

$$
[x, y]^{z}=[\sqrt{-1} x, \sqrt{-1} y]^{z} \quad x, y \in D_{z}^{\circ}
$$

Definition 2.4.2. The notation being as above, the Levi-Tanaka form at $z \in M$ is the $\Xi_{z}$-hermitian form $f_{z}$ on $D_{z}$ defined by

$$
\left.f_{z}(x, y)=-\frac{1}{4}\left([x, \sqrt{-1} y]^{z}-\sqrt{-1}[x, y]^{z}\right) .{ }^{3}\right)
$$

That this is actually $\boldsymbol{\Xi}_{z}$-hermitian, follows from (2.4.1).

Definition 2.4.3. A $W$-hermitian form $f$ on $V$ is said to satisfy the condition $\alpha_{q}$ if and only if, for every $0 \neq \xi \in W^{*}$, one can find, either a subspace of $V$ of dimension at least $q+1$ on which the Hermitian form

3) In this formula, the former $\sqrt{-1}$ is the tensor field induced from the complex structure whereas the latter expresses the multiplication of $\sqrt{-1} \in \mathbf{C}$ in the complexification $\Xi_{z}^{e}$. 
$h(\xi)(x, y)=\langle\xi| f(x, y)>x, y \in V$ is positive definite, or a subspace of $V$ of dimension at least $n-q+1$ on which $h(\xi)$ is negative definite. The real submanifold $M$ is said to satisfy the condition $\alpha_{q}$ (resp. the condition $\nu_{q}$ ) if and only if for each $z \in M, f_{z}$ satisfies the condition $\alpha_{q}$ (resp. the condition $\nu_{q}$ in the sense of Definition 2.3.3).

The following lemma will clarify the implication relation between the conditions $\alpha_{q}, \nu_{q} \quad q=1,2, \ldots n$.

Lemma 2.4.4. If the condition $\nu_{q}$ is satisfied, then $2(q+1) \leqq n$. The condition $\alpha_{q}$ is satisfied if and only if the condition $\alpha_{n-q}$ is satisfied. The condition $\nu_{q}$ is satisfied if and only if the condition $\alpha_{p}$ is satisfied for each $0 \leqq p \leqq q$.

Now we introduce a Hermitian structure $<,>$ on the vector bundle $D^{\circ}$. Then each fiber $D_{z}^{\circ}$ is an $n$-dimensional Hilbert space. We set $h_{z}(\xi)(x, y)=\left\langle\xi \mid f_{z}(x, y)\right\rangle, x, y \in D_{z}^{\circ}, \xi \in \Xi_{z}^{*}$ to define the Hermitian form $h_{z}(\xi)$ on $D_{z}^{\circ}$, and we define the function $\tilde{c}_{q}$ over $\Xi^{*}$ by setting

$$
\tilde{c}_{q}(\xi)=c_{q}^{*}\left(h_{z}(\xi)\right)
$$

where we have defined the right hand side by (2.2.4). By (2) of Lemma 2.2.5, $M$ satisfies the condition $\alpha_{q}$ if and only if $\tilde{c}_{q}>0$ everywhere on $E^{*} \backslash 0$ ( $E^{*}$ minus the zero section). Thus, if $M$ satisfies the condition $\alpha_{q}$, then, according to Theorem 2.2.4 and (8) of Lemma 2.2.5, there is a constant $C_{K}$, for every compact subset $K$ of $\Xi^{*} \backslash 0$, such that

$$
\begin{aligned}
\int|u|^{2} d s \leqq C_{K} \int\left(\left|\bar{\partial}_{\left(h_{z}(\xi)\right)} u\right|^{2}+\left|\delta_{\left(h_{z}(\xi)\right)}^{\circ} u\right|^{2}\right) d s \\
\\
\xi \in K \cap \Xi_{z}, u \in C_{0}^{\infty}\left(D_{z} \times \Xi_{z}, \Lambda_{q} D_{z}^{*}\right)
\end{aligned}
$$

where $d s$ is a translation invariant measure on $D_{z} \times E_{z}$ and the differential operators $\bar{\partial}_{\left(h_{z}(\xi)\right)}, \delta_{\left(h_{z}(\xi)\right)}^{\circ}$ on $D_{z}$ should be regarded also as differential operators on $D_{z}^{\circ} \times \Xi_{z}$ in trivial fashion. By a theorem in Hörmander [6], we see that (2.4.2) implies that the Dolbeault sequences for $M$ are subelliptic at their $(q+1)$-th terms provided $M$ satisfies the condition $\alpha_{q}$. 
To make this precise, note first that the Hermitian structure $\left\langle,>\right.$ on $D^{\circ}$ naturally induces the Hermitian structure $<,>$ on each $D^{(0, q)}, 0 \leqq q \leqq n$ through the canonical anti-isomorphism between $\Lambda^{q} D^{\circ *}$ and $D^{(0, q)}$ (Lemma 2.2.1). Choosing a measure $d v$ on $M$, we can thus define the norm $\|u\|_{1}=\left(\int\langle u, u\rangle d v\right)^{\frac{1}{2}}$ for sections $u$ of $D^{(0, q)}, 0 \leqq q \leqq n$. We now introduce Sobolev norms \|\|$_{(s)}, s \in \mathbf{R}$ for sections of the vector bundles $D^{(0, q)}$, $0 \leqq q \leqq n$. (Usually these norms for sections of a vector bundle $E$ over $M$ are defined in the following way: Take a locally finite coordinate covering $\left\{\omega_{\lambda}\right\}$ over $M$, and choose a partition of unity $\left\{\varphi_{\lambda}\right\}\left(\varphi_{\lambda} \in C_{0}^{\infty}\left(\omega_{\lambda}\right)\right)$ and local frames $s_{\lambda}^{1}, \ldots, s_{\lambda}^{e} \in C^{\infty}\left(\omega_{\lambda}, E\right)(e=$ fiber-dim $E)$. We set $\varphi_{\lambda} u=$ $\sum_{i=1}^{e} u_{\lambda, i} s_{\lambda}^{i}$ for $u \in C_{0}^{\infty}(E)$ to define $u_{\lambda, i} \in C_{0}^{\infty}\left(\omega_{\lambda}\right)$, further we define \|\|$_{s}$ by

$$
\|u\|_{(s)}^{2}=\sum_{\lambda} \sum_{i=1}^{e}\left\|u_{\lambda, i}\right\|_{(s)}^{2}
$$

regarding $\omega_{\lambda}$ as situated in $\mathbf{R}^{m}$ and $u_{\lambda, i}$ as an element of $C_{0}^{\infty}\left(\mathbf{R}^{m}\right)(m=$ $\left.\operatorname{dim}_{\mathbb{R}} M\right)$. The norm \|\|$_{(s)}$ is not unique but the passage to another coordinate covering, or another partition of unity, or another local frame gives also an equivalent norm on $C_{0}^{\infty}(K, E)$ for every compact subset $K$ of $M$.)

Theorem 2.4.5. The notation being as above, define the adjoint $\delta_{b}$ of the differentiation $\bar{\partial}_{b}: C^{\infty}\left(D^{(0, q-1)}\right) \rightarrow C^{\infty}\left(D^{(0, q)}\right)$ of the Dolbeault sequence $D^{(0, \cdot)}$ for $M$, by

$$
\int\left\langle\bar{\partial}_{b} u, v>d v=\int\left\langle u, \delta_{b} v>d v \quad u \in C_{0}^{\infty}\left(D^{(0, q-1)}\right), v \in C_{0}^{\infty}\left(D^{(0, q)}\right) .\right.\right.
$$

If $M$ satisfies the condition $\alpha_{q}$, then the differential operator

$$
C_{0}^{\infty}\left(D^{(0, q)}\right) \ni u \rightarrow\left(\bar{\partial}_{b} u, \delta_{b} u\right) \in C_{0}^{\infty}\left(D^{(0, q+1)} \oplus D^{(0, q-1)}\right)
$$

is subelliptic, that is, for every compact subset $K$ of $M$ there is a constant $C_{K}>0$ such that

$$
\|u\|_{\left(\begin{array}{l}
1 \\
2
\end{array}\right)}^{2} \leqq\left(\left\|\bar{\partial}_{b} u\right\|^{2}+\left\|\delta_{b} u\right\|^{2}+\|u\|^{2}\right) \quad u \in C_{0}^{\infty}\left(K, D^{(0, q)}\right) .
$$


By Theorem 1.4.2 in Hörmander [6] we can reduce (2.4.3) to (2.4.2) with $d s$ replaced $d v_{z} ; d v_{z}$ can be regarded as a translation invariant measure on $T_{z}(M)$, hence also it is regarded as a translation invariant measure on $D_{z} \times \Xi_{z}$ by the short exact sequence $0 \rightarrow D_{z} \rightarrow T_{z}(M) \rightarrow \Xi_{z} \rightarrow 0$.

Remark. The estimate (2.4.3) implies the subellipticity of the generalized Laplacian $C_{0}^{\infty}\left(D^{(0, q)}\right) \ni u \longrightarrow\left(\bar{\partial}_{b} \delta_{b}+\delta_{b} \bar{\partial}_{b}\right) u \in C_{0}^{\infty}\left(D^{(0, q)}\right)$. In fact we can prove the following by using the method of Hörmander [7]: Let $E, F_{i} i=1,2, \ldots, s$ be Hermitian vector bundles over a manifold $M$ and let $X_{i}: E \rightarrow F_{i}, 1 \leqq i \leqq s$ be first order differential operators. Assume that for each compact subset $K$ of $M$ one can find constants $\varepsilon>0, C>0$ such that

$$
\|u\|_{(\varepsilon)} \leqq C\left(\sum_{i=1}^{s}\left\|X_{i} u\right\|_{(0)}+\|u\|_{(0)}\right) \quad u \in C_{0}^{\infty}(K, E)
$$

Then the operator $\sum_{i=1}^{s} X_{i}{ }^{*} X_{i}$ is hypoelliptic where $X_{i}^{*}$ is the adjoint of $X_{i}$ with respect to the Hermitian structures of $E$ and $F_{i}$. In $[7] E, F_{i}$ were assumed to be the trivial bundle of rank 1 and $X_{i}$ to be real. The present generalization, however, leads to no change of the argument [7] except a few obvious modifications.

Finally we mention a lemma which computes the Levi-Tanaka form of the standard real submanifold $M_{f}$, where $f$ is a $W$-hermitian form on $V$. Recall that $M_{f}$ is a Lie group whose left multiplications are extended to affine transformations of $V \times W^{c}$. Therefore we may compute only the Levi-Tanaka form at 0 . As done before, $T_{0}\left(M_{f}\right)$ can be naturally identified with $V \times W$ by identifying $T_{0}\left(V \times W^{c}\right)$ with $V \times W^{c}$. Let $D^{\circ}=T\left(M_{f}\right) \cap \sqrt{-1} T\left(M_{f}\right)$ and let $\Xi=T(M) / D^{\circ}$. Then $D_{0}^{\circ}=(V \times W) \wedge$ $\sqrt{-1}(V \times W)=V$, and $\Xi_{0}=V \times W / V$, by which we identify $\Xi_{0}$ with $W$.

Lemma 2.4.6. Under the above identification $f$ is identical with the Levi-Tanaka form of $M_{f}$ at the origin. Thus $M_{f}$ satisfies the condition $\alpha_{q}$ (resp. the condition $\nu_{q}$ ) if and only if $f$ satisfies the condition $\alpha_{q}$ (resp. the condition $\nu_{q}$ ). 
We will give the proof of Lemma 2.4.6 in Appendix 1.

§ 2.5. Exactness theorem for the Dolbeault sequences. The main object of this section is to prove the following

Theorem 2.5.1. Let $M$ be a generic real submanifold of the second kind, and denote the $p$-th Dolbeault sequence of $M$ by

$$
0 \longrightarrow D^{(p, 0)} \stackrel{\bar{\partial}_{b}}{\longrightarrow} D^{(p, 1)} \longrightarrow \cdots \stackrel{\bar{\partial}_{b}}{\longrightarrow} D^{(p, n)} \longrightarrow 0 .
$$

If $M$ satisfies the condition $\nu_{q_{0}}$, then one can find for every $z \in M$ an arbitrarily small neighbourhood $U$ such that the following sequences are exact:

$$
\begin{aligned}
& 0 \rightarrow C_{0}^{\infty}\left(U, D^{(p, 0)}\right) \rightarrow C_{0}^{\infty}\left(U, D^{(p, 1)}\right) \rightarrow \cdots \rightarrow C_{0}^{\infty}\left(U, D^{\left(p, q_{0}+1\right)}\right) \\
& \Gamma\left(\bar{U}, \underline{D}^{\left(p, n-q_{0}-1\right)}\right) \rightarrow \Gamma\left(\bar{U}, \underline{D}^{\left(p, n-q_{0}\right)}\right) \rightarrow \cdots \rightarrow \Gamma\left(\bar{U}, \underline{D}^{(p, n)}\right) \rightarrow 0 .
\end{aligned}
$$

We will prove this by combining Theorem 2.2.1 and Theorem 2.4.5. Before proceeding we require a lemma which allows us to approximate locally an arbitrary real submanifold of the second kind by a suitable standard one.

Lemma 2.5.2. Let $M$ be a real submanifold of the second kind and let $D^{\circ}=T(M) \cap \sqrt{-1} T(M), \Xi=T(M) / D^{\circ}$. Let further $f_{z}$ be the LeviTanaka form at $z \in M$. Then there is an analytic diffeomorphism $\sigma$ from a neighbourhood $U$ in $X$ of $z$ into the complex vector space $D_{z}^{\circ} \times \Xi_{z}^{c}$ satisfying the following conditions:

(1) $\sigma(z)=0$.

(2) $d \sigma_{z} \mid D_{z}^{\circ}=1_{D_{z}^{\circ} \text {. }}$

(3) There is a neighbourhood $\Omega$ of 0 in $D_{z}^{\circ} \times E_{z}$ and a smooth map $\rho: \Omega \rightarrow \Xi_{z}$ such that

a) $\rho(y, x)\left(y \in D_{z}^{\circ}, x \in E_{z},(y, x) \in \Omega\right)$ vanishes at 0 to order $O\left(|y|^{3}+|y||x|+|x|^{2}\right)$

b) $\sigma(M \cap U)=\left\{(y, x+\sqrt{-1}(f z(y, y)+\rho(y, x))) \in D_{2}^{\circ} \times \Xi_{z}^{c} \mid(y, x)\right.$ $\in \Omega\}$. 
This lemma follows easily from the implicit function theorem, we give the proof for this in Appendix 1, not to violate the continuity. (See also Greenfield [3].)

Now we assume that $M$ can be written in the form

$$
M=\left\{\left(z, x+\sqrt{-1}(f(z, z)+\rho(z, x)) \in V \times W^{c} \mid(z, x) \in V \times W\right\}\right.
$$

where $f$ is a $W$-hermitian form on $V$ satisfying the condition $\nu_{q_{0}}$ and $\rho$ is a smooth $W$-valued map of $V \times W$ vanishing to the order $O\left(|z|^{3}+|z|\right.$ $|x|+|x|^{2}$ ) at the origin. This is actually no loss of generality in proving Theorem 2.5.1 in view of Lemma 2.5.2; one may only extend the function $\rho$ in the lemma to the whole space $D_{2}^{\circ} \times \Xi_{z}$ by changing $\rho$ outside a neighbourhood of 0 . It suffices to prove the conclusion of Theorem 2.5.1 with $M$ of this form and for $z=0$. Take arbitrary $x \in C_{0}^{\infty}(V \times W)$ such that $\chi=1$ in a neighbourhood of the origin and set for $a \in \mathbf{R}$

$$
\begin{aligned}
& M_{0}=M_{f}=\{(z, x+\sqrt{-1} f(z, z)) \mid(z, x) \in V \times W\} \\
& M_{a}=\left\{\left(z, x+\sqrt{-1}\left(f(z, z)+a^{-2} \chi(z, x) \rho\left(a z, a^{2} x\right)\right)\right) \mid\right. \\
& (z, x) \in V \times W\} \quad \text { if } a \neq 0 .
\end{aligned}
$$

Then there is a neighbourhood of 0 in $M$ and a neighbourhood of 0 in $M_{a}(a \neq 0)$ which are mutually mapped onto the other by the analytic correspondence $(z, x) \leftrightarrow\left(a z, a^{2} x\right)$. Thus it suffices to prove the conclusion of Theorem 2.5.1 with $M$ replaced by $M_{a}$ and for $z=0$. Note that the hypothesis $\rho(z, x)=O\left(|z|^{3}+|z||x|+|x|^{2}\right)$ implies that $\rho_{a}(z, x)=a^{-2}$ $\chi(z, x) \rho\left(a z, a^{2} x\right)$ converges to 0 in the topology of $C_{0}^{\infty}\left(K_{0}, W\right)$ when $a \rightarrow 0$, where $K_{0}=\operatorname{supp} x$. Thus the submanifold $M_{a}$ might be said to converge to the standard real submanifold $M_{0}=M_{f}$ when $a \rightarrow 0$. (Note also that the dependence of $M_{a}$ on $a \in \mathbf{R}$ is smooth.)

Choose now a base $\left\{e_{1}, \ldots, e_{n}\right\}$ (over $\mathbf{C}$ ) in $V$ and a base $\left\{e_{1}^{\prime}, \ldots\right.$, $\left.e_{d}^{\prime}\right\}$ (over $\mathbf{R}$ ) in $W$, and introduce the coordinate system $\left(z_{1}, \ldots, z_{N}\right), N=$ $n+d$ in $V \times W^{c}$ by setting $z=\sum_{i=1}^{n} z_{i} e_{i}+\sum_{i=1}^{d} z_{i+n} e_{i}^{\prime} z \in V \times W^{c}$. Then, if $|a|$ is sufficiently small, the real submanifold $M_{a}$ is still generically situated with respect to the coordinate system $\left(z_{1}, \ldots, z_{N}\right)$. (See Definition 
2.1.2.) By this coordinate system we shall trivialize the 0 -th Dolbeault sequence of $M_{a}$

$$
0 \longrightarrow D_{a}^{(0,0)} \stackrel{\bar{\partial}_{a}}{\longrightarrow} D_{a}^{(0,1)} \stackrel{\bar{\partial}_{a}}{\longrightarrow} \cdots \stackrel{\bar{\partial}_{a}}{\longrightarrow} D_{a}^{(0, n)} \longrightarrow 0
$$

as done at the end of $\S 2.1$; the resulting complex we denote by

$$
0 \longrightarrow E_{a}^{0} \stackrel{\bar{\partial}_{a}}{\longrightarrow} E_{a}^{1} \stackrel{\bar{\partial}_{a}}{\longrightarrow} \cdots \stackrel{\bar{\partial}_{a}}{\longrightarrow} 0
$$

where $E_{a}^{q}$ denotes the trivial bundle of $\operatorname{rank}\left(\begin{array}{c}n \\ q\end{array}\right)$ over $M_{a}$. Identifying each $M_{a}$ with $V \times W$ by the diffeomorphism $V \times W \ni(z, x) \rightarrow(z, x+\sqrt{-1}$ $\left.\left(f(z, z)+\rho_{a}(z, x)\right)\right) \in M_{a}\left(V \times W^{c}\right.$, we can regard $E_{a}^{q}, 0 \leqq q \leqq n$ as the trivial bundles $E^{q}$ of $\operatorname{rank}\left(\begin{array}{l}n \\ q\end{array}\right)$ over the vector space $V \times W$. We thus have defined the complex of the differential operators $\bar{\partial}_{a}$ depending on a parameter $a$ (belonging some interval $|a| \leqq a_{0}$ ) acting the same vector bundles $E^{q}, 0 \leqq q \leqq n$

$$
0 \longrightarrow E^{0} \stackrel{\bar{\partial}_{a}}{\longrightarrow} E^{1} \longrightarrow \cdots \stackrel{\bar{\partial}_{a}}{\longrightarrow} E^{n} \longrightarrow 0
$$

and we shall denote this complex by $E_{a}^{\cdot}$ hereafter. As often done so far, we interpret a smooth section of $E^{q}$ as an alternating function depending on $q$-tuples from $\{1,2, \ldots, n\}$, and with values in $C^{\infty}(V \times W)$. (See the discussion after Definition 2.1.2.) Recall that, if we define the first order differential operators $\bar{\partial}_{a, k}, 1 \leqq k \leqq n$ by

$$
\bar{\partial}_{a} u=\left(\bar{\partial}_{a, 1} u, \bar{\partial}_{a, 2} u, \ldots, \bar{\partial}_{a, n} u\right) \quad u \in C^{\infty}(M),
$$

then $\bar{\partial}_{a} u$ for $u \in C^{\infty}\left(E_{b}\right), 1 \leqq q \leqq n$ are given by replacing $\bar{\partial}_{b} u, \bar{\partial}_{k}$ by $\bar{\partial}_{a} u, \bar{\partial}_{a, k}$ in (2.1.1). A simple computation shows

$$
\bar{\partial}_{0, k}=\frac{\partial}{\partial \bar{z}_{k}}-\sqrt{-1} \sum_{i=1}^{d} \sum_{j=1}^{n} h_{k j}^{i} z_{j} \frac{\partial}{\partial x_{i}}
$$

where the Hermitian forms $h^{i}(z, z)=\sum_{j, k} h_{j k}^{i} z_{j} \bar{z}_{k}, 1 \leqq i \leqq d$ are defined by $f(z, z)=\sum_{i=1}^{d} h^{i}(z, z) e_{i}^{\prime}$. Set 


$$
\begin{aligned}
<u, v> & =\sum_{|K|=q} u_{K} \overline{v_{K}} \quad u, v \in C_{0}^{\infty}\left(V \times W, E^{q}\right) \\
|u|^{2} & =<u, u>.
\end{aligned}
$$

We introduce a one-parameter family of measures on $V \times W$ setting

$$
d V_{t}=(2 \pi \sqrt{-1})^{-n} e^{-t|z|^{2}} d z_{1} d \bar{z}_{1} \ldots d z_{n} d \bar{z}_{n} d x_{1} d x_{2} \ldots d x_{d}
$$

and define the adjoints $\delta_{a}^{t}$ of $\bar{\partial}_{a}$ by

$$
\begin{aligned}
\int<\bar{\partial}_{a} u, v>d V_{t} & =\int\left\langle u, \delta_{a}^{t} v>d V_{t}\right. \\
& u \in C_{0}^{\infty}\left(V \times W, E^{q-1}\right), v \in C_{0}^{\infty}\left(V \times W, E^{q}\right) .
\end{aligned}
$$

Now the proof of Theorem 2.5.1 follows essentially from the following

Lemma 2.5.3. If $t>0$ (resp. $t<0$ ), then there is a constant $a_{0}>0$ and a constant $C_{t}>0$ such that

$$
\int|u|^{2} d V_{t} \leqq C_{t} \int\left(\left|\bar{\partial}_{a} u\right|^{2}+\left|\delta_{a}^{t} u\right|^{2}\right) d V_{t} \quad u \in C_{0}^{\infty}\left(V \times W, E^{q}\right)
$$

if $n-q_{0} \leqq q \leqq n$ (resp. $0 \leqq q \leqq q_{0}$ ) and if $|a|<a_{0}$.

Proof. a) Case $t>0, n-q_{0} \leqq q \leqq n$. Note $\bar{\partial}_{0}=\bar{\partial}_{f}$ in the notation of $\S 2.3$. Hence the assumption that $f$ satisfies the condition $\nu_{q_{0}}$ implies $c_{q}(f)>0$. Therefore applying Theorem 2.3.2, we find a constant $C>0$ such that

$$
t \int|u|^{2} d V_{t} \leqq C \int\left(\left|\delta_{0} u\right|^{2}+\left|\delta_{0}^{t} u\right|^{2}\right) d V_{t} \quad u \in C_{0}^{\infty}\left(E^{q}\right)
$$

Now let $K_{0}$ denote the support of $\chi$ (see (2.5.1)), and choose another compact $K_{1}$ which contains $K_{0}$ in its interior and choose $\varphi \in C^{\infty}(V \times W)$ so that $\varphi=1$ outside $K_{1}, \varphi=0$ in a neighbourhood of $K_{0}$, and $0 \leqq \varphi \leqq 1$ everywhere. Since $\bar{\partial}_{a}, \delta_{a}^{t}$ coincide with $\bar{\partial}_{0}, \delta_{0}^{t}$ outside $K_{0}$, we obtain from (2.5.4)

$$
t \int|\varphi u|^{2} d V_{t} \leqq C \int\left(\left|\bar{\partial}_{a}(\varphi u)\right|^{2}+\left|\delta_{a}^{t}(\varphi u)\right|^{2}\right) d V_{t} \quad u \in C_{0}^{\infty}\left(E^{q}\right)
$$


We set $\vartheta_{a}(\varphi) u=\varphi \bar{\partial}_{a} u-\bar{\partial}_{a}(\varphi u)$. Then $\vartheta_{a}(\varphi)$ is of order 0 , and $\vartheta_{a}(\varphi)^{*} u$ $=\varphi \delta_{a}^{t} u-\delta_{a}^{t}(\varphi u)$ where $\vartheta_{a}(\varphi)^{*}$ denotes the conjugate transposed of $\vartheta_{a}(\varphi)$ when $\vartheta_{a}(\varphi)$ regarded as a matrix of scalar operators. Since $\vartheta_{a}(\varphi)=0$ outside $K_{1}$, we can find a constant $C$ such that

$$
\begin{gathered}
t \int|\varphi u|^{2} d V_{t} \leqq C\left(\int\left(\left|\bar{\partial}_{a} u\right|^{2}+\left|\delta_{a}^{t} u\right|^{2}\right) d V_{t}+\int_{K_{1} \backslash K_{0}}|u| d V_{t}\right. \\
u \in C_{0}^{\infty}\left(E^{q}\right) .
\end{gathered}
$$

Now choose $\phi \in C_{0}^{\infty}(V \times W)$ so that $\phi=1$ in a neighbourhood of $K_{1}$. We will estimate $\|\psi u\|_{(1 / 2)}^{2}$ by the right hand side of (2.5.5) plus $\int|u|^{2} d V_{t}$ and times a positive constant, when $|a|$ is sufficiently small. Note that the condition $\nu_{q_{0}}$ is stable under small perturbation of real submanifolds, so that there is a constant $a^{\prime}>0$ such that $M_{a},|a| \leqq a^{\prime}$ still satisfies the condition $\nu_{q_{0}}$. Further, note that the condition $\nu_{q_{0}}$ implies the conditions $\alpha_{q} n-q_{0} \leqq q \leqq n$. Hence, by Theorem 2.4.1, we obtain a constant $C_{t}$, depending only on $t>0$, such that, when $|a| \leqq a^{\prime}$

$$
\begin{aligned}
& \|\psi u\|_{\left(\begin{array}{l}
1 \\
2
\end{array}\right)}^{2} \leqq C_{t}\left(\int\left(\left|\bar{\partial}_{a} u\right|^{2}+\left|\delta_{t}^{a} u\right|^{2}\right) d V_{t}+\int|u|^{2} d V_{t}\right) \\
& u \in C_{0}^{\infty}\left(E^{q}\right) .
\end{aligned}
$$

(The independence of this $C_{t}$ on the parameter $a \in\left[-a^{\prime}, a^{\prime}\right]$ can be checked directly by analyzing the rather long course of the proof of Theorem 1.4.2 of [6] which we rely on when stating Theorem 2.4.1.)

Now let us prove (2.5.3) when $|a|$ is sufficiently small. Assume the converse is true, that is, there is a sequence $a_{\nu}$ such that $a_{\nu} \rightarrow 0(\nu \rightarrow \infty)$, and a sequence $u_{\nu} \in C_{0}^{\infty}\left(E^{q}\right)$ such that $\int\left|u_{\nu}\right|^{2} d V_{t}=1$ whereas $\int\left(\left|\bar{\partial}_{a_{\nu}} u\right|^{2}\right.$ $\left.+\left|\delta_{a_{\nu}}^{t} u\right|^{2}\right) d V_{t} \rightarrow 0(\nu \rightarrow \infty)$. Then $\left\|\psi u_{\nu}\right\|_{(1 / 2)}$ is bounded by (2.5.6), so the generalized Relich lemma implies that the sequence $u_{\nu} \mid K_{1}, \nu=1,2, \ldots$ is precompact in $L^{2}\left(K_{1}\right)$. Thus we could assume from the beginning that $\int_{K_{1}}\left|u_{\mu}-u_{\nu}\right|^{2} d V_{t} \rightarrow 0 \mu$, when $\nu \rightarrow \infty$. But this, combined with (2.5.5) implies $\int\left|u_{\mu}-u_{\nu}\right|^{2} d V_{t} \rightarrow 0$, that is, $u_{\nu}$ is a Cauchy sequence. Denote by $u_{0}$ the limit of this sequence. Since $\int\left(\left|\bar{\partial}_{a_{\nu}} u_{\nu}\right|^{2}+\left|\delta_{a_{\nu}}^{t} u_{\nu}\right|^{2}\right) d V_{t} \rightarrow 0, \bar{\partial}_{0} u_{0}$ 
and $\delta_{0}^{t} u_{0}$, defined in the distribution sense, both vanish. Since the operator $C_{0}^{\infty}\left(E^{q}\right) \ni u \rightarrow\left(\bar{\partial}_{0} u, \delta_{0}^{t} u\right) \in C_{0}^{\infty}\left(E^{q+1} \oplus E^{q-1}\right)$ is subelliptic by Theorem 2.4.1, it follows $u_{0}$ is smooth. Now we introduce a sequence $\eta_{\nu} \in C_{0}^{\infty}(V \times W)$, $\nu=1,2, \cdots$ by setting

$$
\eta_{\nu}(z, x)=\eta_{0}\left(z / \nu, x / \nu^{2}\right) \quad(z, x) \in V \times W
$$

where $\eta_{0} \in C_{0}^{\infty}(V \times W)$ equals 1 in a neighbourhood of 0 . If $P(D)$ is a differential operator of the form

$$
\sum_{i} c_{i} \frac{\partial}{\partial \overline{z_{i}}}+\sum_{j, k} c_{j k} z_{j} \frac{\partial}{\partial x_{k}}
$$

then $P(D) \eta_{\nu}(z, x)=\left(P(D) \eta_{0}\right)\left(z / \nu, x / \nu^{2}\right)$ so that $\left|P(D) \eta_{\nu}\right| \leqq C$ for some $C>0$ not depending on $\nu$. Therefore, in view of (2.5.2) and (2.1.1), the entries of the matrix $\vartheta\left(\eta_{\nu}\right)$, defined by $\vartheta\left(\eta_{\nu}\right) u=\eta_{\nu} \bar{\partial}_{0} u-\bar{\partial}_{0}\left(\eta_{\nu} u\right) u \in$ $C_{0}^{\infty}\left(E^{q}\right)$, are bounded in absolute value by a constant $C>0$ not depending on $\nu$. Thus $\left|\vartheta\left(\eta_{\nu}\right) u\right| \leqq C|u| u \in C_{0}^{\infty}\left(E^{q}\right)$ for some constant $C$ not depending on $\nu$. Since $\bar{\partial}_{0}\left(\eta_{\nu} u_{0}\right)=-\vartheta\left(\eta_{\nu}\right) u_{0}, \delta_{0}^{t}\left(\eta_{\nu} u_{0}\right)=-\vartheta\left(\eta_{\nu}\right)^{*} u_{0}$, the dominated convergence theorem implies

$$
\int\left(\left|\bar{\partial}_{0}\left(\eta_{\nu} u\right)\right|^{2}+\left|\delta_{0}^{t}\left(\eta_{\nu} u\right)\right|^{2}\right) d V_{t} \rightarrow 0 \quad(\nu \rightarrow \infty),
$$

from which we further obtain by applying (2.5.4)

$$
\int\left|\eta_{\nu} u_{0}\right|^{2} d V_{t} \rightarrow 0 \quad(\nu \rightarrow \infty)
$$

But $\int\left|u_{0}\right|^{2} d V_{t}=1$ since $\int\left|u_{\nu}\right|^{2} d V_{t}=1 \quad \nu=1,2, \ldots$ This contradiction completes the proof in case $t>0, n-q_{0} \leqq q \leqq n$.

b) Case $t<0,0 \leqq q \leqq q_{0}$. Similarly proved.

Q.E.D.

Now we shall denote by $E_{a}$ the complex

$$
0 \longrightarrow E^{0} \stackrel{\bar{\partial}_{a}}{\longrightarrow} E^{1} \stackrel{\bar{\partial}_{a}}{\longrightarrow} \cdots \stackrel{\bar{\partial}_{a}}{\longrightarrow} E^{n} \longrightarrow 0 .
$$

Proof of Theorem 2.5.1. Note that, by the sequence $\eta_{\nu} \nu=1,2, \ldots$ 
having appeared in the above proof, the condition $(*)$ in $\S 1.4$ is fulfilled with $E^{\cdot}$ replaced by $E_{0}$, hence also with $E^{\cdot}$ replaced by $E_{a}^{\cdot},|a| \leqq a_{0}$ because $E_{0}$ and $E_{a}^{*}$ are the same outside $K_{0}=\operatorname{supp} \chi$. Further, by Lemma 2.5.3 and the remark after Theorem 2.4.5, we conclude that the assumption $\mathscr{B}_{n-q_{0}}$ and $\mathscr{A}_{q_{0}}$ in $\S 1.4$ are satisfied for $E_{a}^{\cdot},|a| \leqq a_{0}$ if one takes $d V_{t}, t>0$ or $d V_{t}, t<0$ as $d v$ in $\S 1.4$.

Now our next aim here is to find a subring of the ring of $E_{a}^{\cdot}$-analytic functions which is well filtered in the sense of Definition 1.4.4. Let $P$ be the set of (holomorphic) polynomial functions over $V \times W^{c}$. We define for each element $f \in P$ unusual degree $d(f)$ by the following rules

(i ) $d(f)=0$ if and only if $f$ is a constant.

(ii) $\quad d\left(z_{i}\right)=1 \quad i=1,2, \cdots n$, whereas $d\left(z_{i}\right)=2$ if $n<i \leqq N$.

(iii) $d(f g)=d(f)+d(g)$ provided $f g \neq 0$.

Then we obtain filtration $P_{0} \subset P^{1} \subset P^{2} \cdots$ setting $P^{k}=\{f \in P \mid d(f) \leqq k\}$. Denoting the map $V \times W \ni(z, x) \rightarrow\left(z, x+\sqrt{-1}\left(f(z, z)+\rho_{a}(z, x)\right)\right) \in V \times$ $W^{c}$ by $\iota_{a}$, we define a ring of $E_{a}^{\cdot}$-analytic functions $P_{a}$ as the pull back $\iota_{a}^{*}(P)$ of $P$, that is, $P_{a}=\left\{f \circ \iota_{a}=\iota_{a}^{*}(f) \mid f \in P\right\}$. We set also $P_{a}^{k}=\iota_{a}^{*}\left(P^{k}\right)$. Choose a smooth function $\varphi_{0}$ in $W$, which vanish nowhere in $W$, such that $\varphi_{0}(x)=|x|=\sqrt{x_{1}^{2}+\cdots x_{d}^{2}}$ outside a compact subset, and set

$$
\chi_{\nu}(z, x)=e^{-\nu^{-1} \nu^{\prime}} \overline{|z|^{2}+\varphi_{0}(x)} \text {. }
$$

Lemma 2.5.4. The ascending chain $P_{a}^{0} \subset P_{a}^{1} \subset \cdots\left(|a| \leqq a_{0}\right)$, together with the sequence $x_{\nu} \nu=1,2, \ldots$, satisfies the condition of Definition 1.4.4 when $E^{\cdot}$ replaced by $E_{a}^{\cdot}$, that is, the ring $P_{a}$ is well filtered for $E_{a}^{\cdot}$.

Note that the Hermitian structures on $E^{q}, 0 \leqq q \leqq n$ are already defined by the trivialization relative to the coordinates $\left(z_{1}, \ldots, z_{n}\right)$.

It suffices to prove this lemma when $a=0$, since the complexes $E_{a}$. coincide with $E_{0}$ outside a compact subset. But the case $a=0$ can be checked directly in view of (2.5.2) and (2.1.1). Thus the combination of Theorem 1.4.5 and of Corollaries 1.3.1 and 1.3.2 proves that the conclusion of Theorem 2.5.1 is true for $z=0$ if $M=M_{a},|a| \leqq\left|a_{0}\right|$. (Note that all domains of $M_{a}$ of the form $P(f)$, where $f$ is a family of a finite number of elements of $P_{a}$, form a fundamental system of neighbourhoods 
of $M_{a}$.) As was remarked after Theorem 2.5.1, this completes the proof of this theorem.

Note that $M_{f}=M_{0}$ in the above proof. Thus we have also proved the following, more satisfactory result for standard real submanifolds.

Theorem 2.5.5. Let a W-hermitian form $f$ on $V$ satisfy the condition $\nu_{q_{0}}$. If an open subset $U$ of $M_{f}$ can be written as an intersection of $M_{f}$ with some polynomial polyhedron of $V \times W^{c}$, then the sequence

$$
0 \rightarrow C_{0}^{\infty}\left(U, D_{f}^{(p, 0)}\right) \rightarrow C_{0}^{\infty}\left(U, D_{f}^{(p, 1)}\right) \rightarrow \cdots \rightarrow C_{0}^{\infty}\left(U, D_{f}^{\left(p, q_{0}+1\right)}\right)
$$

is exact. If a compact subset $K$ of $M_{f}$ is written as an intersection of $M_{f}$ with some polynomially convex compact subset of $V \times W^{c}$, the sequence

$$
\Gamma\left(K, D_{f}^{\left(p, n-q_{0}-1\right)}\right) \rightarrow \Gamma\left(K, D_{f}^{\left(p, n-q_{0}\right)}\right) \rightarrow \cdots \rightarrow \Gamma\left(K, D_{f}^{(p, n)}\right) \rightarrow 0
$$

is exact.

Finally we state as an application of Theorem 2.5.5, a continuation theorem of Hartogs-Bochner type. We begin with an easy consequence of Theorem A of p. 184 of Naruki [11]:

Lemma 2.5.6. Let $f$ be a $W$-hermitian form on $V$. If a smooth function $u$, defined in a domain $U$ of $M_{f}$, satisfies the tangential CauchyRiemann equation $\bar{\partial}_{b} u=0$, and if $u=0$ in some non-empty open subset of $U$, then $u=0$ identically in $U$.

Now our continuation theorem is stated as follows:

Theorem 2.5.7. Let a $W$-hermitian form $f$ on $V$ satisfy the condition $\nu_{2}$. Let $\Omega$ be a relatively compact domain of $M_{f}$ whose boundary $\partial \Omega$ is also a generic real submanifold of $V \times W^{c}$. Then every smooth function $u$ in $\partial \Omega$ satisfying the tangential Cauchy-Riemann equation of $\partial \Omega$, can be extended uniquely to $v \in C^{\infty}(\bar{\Omega})$ such that $v \mid \Omega$ satisfies the tangential Cauchy-Riemann equation of $\Omega$.

In view of Lemma 2.5.6 and of the exact sequence $C_{0}^{\infty}\left(M_{f}\right)=$ $C_{0}^{\infty}\left(D_{f}^{(0,0)}\right) \rightarrow C_{0}^{\infty}\left(D_{f}^{(0,1)}\right) \rightarrow C_{0}^{\infty}\left(D_{f}^{(0,2)}\right)$, we can prove this lemma following 
entirely the method of Ehrenpreis [1]. See also Theorem 2.3.2' of Hörmander [5].

\section{Appendix 1}

First we prove Lemma 2.4.6. Recall that $M_{f}$ is a Lie group whose identity element is the origin 0 of $V \times W^{c}$. Since the tangent space of $M_{f}$ is naturally identified with $V \times W, V \times W$ is considered to be the Lie algebra of $M_{f}$. By (2.3.1) the curve $(t z, t x+\sqrt{-1} f(z, z)), t \in \mathbf{R}$ in $M_{f}$ is a one-parameter subgroup. Note that the tangent vector of this curve at $t=0$ equals $(z, x) \in V \times W=T_{0}\left(M_{f}\right)$. Thus

$$
\exp (z, x)=(z, x+\sqrt{-1} f(z, z)) \text {. }
$$

Also by (2.3.1) we can compute $\exp t z, \exp t z^{\prime}$ when $z, z^{\prime} \in V(C V \times W)$; that is,

$$
\exp t z \cdot \exp t z^{\prime}=\exp \left(t\left(z+z^{\prime}\right), \sqrt{-1} t^{2}\left(f\left(z^{\prime}, z\right)-f\left(z, z^{\prime}\right)\right)\right) .
$$

Note the following formula which is valid in the Lie group theory:

$$
\exp t X \exp t Y=\exp \left(t(X+Y)+\frac{t^{2}}{2}[X, Y]+\cdots\right)
$$

where the dots indicate the terms of higher order in $t$. Thus we obtain from $(\alpha .1)$

$$
\frac{1}{2}\left[z, z^{\prime}\right]=\sqrt{-1}\left(f\left(z^{\prime}, z\right)-f\left(z, z^{\prime}\right)\right) .
$$

From this it follows

$$
f\left(z, z^{\prime}\right)=\frac{1}{4}\left(-\left[z, \sqrt{-1} z^{\prime}\right]+\sqrt{-1}\left[z, z^{\prime}\right]\right)
$$

which is the conclusion of Lemma 2.4.6.

Proof of Lemma 2.5.2. Since the statement of this lemma is entirely of local nature, we can assume that $X$ is a complex vector space. We can assume that $z$ is the origin 0 of $X$. We identify $X$ with its tangent space 
at 0 . Then $D_{z}^{\circ}$, hereafter denoted by $V$, is a complex subspace of $X$ and $T_{z}(M)$ is a real subspace of $X$ such that $T_{z}(M)+\sqrt{-1} T_{z}(M)=X$. Choose a complementary subspace $W$ of $V$ in $T_{z}(M)$. Then

$$
X=V+W+\sqrt{-1} W=V+W^{c} \text { (the direct sum). }
$$

With this direct decomposition in mind, let $p^{1}, p^{2}$ denote the projections of $X$ onto $T_{z}(M)=V+W, \sqrt{-1} W$ respectively, and let $\iota^{1}, c^{2}$ be the restrictions of $p^{1}, p^{2}$ to the submanifold $M$. Since $V \times W=T_{z}(M)$, the differential $d c_{z}^{1}$ at $z(=0)$ is the identity of $V \times W$. (We shall identify the direct sum of two vector spaces with the cartesian product of them; thus $T_{z}(M)=V+W=V \times W, X=V+W^{c}=V \times W^{c}$.) Thus we obtain by the implicit function theorem an inverse $\tau$ of $c^{1}$ defined in a neighbourhood $\Omega$ of 0 in $V \times W$. The set $\tau(\Omega)$ is a neighbourhood of $z$ in $M$ and

$$
\tau(\Omega)=\left\{\left(z, x+\iota^{2} \cdot \tau(z, x)\right) \in V \times W^{c} \mid(z, x) \in \Omega\right\} .
$$

Now the extraction from $\iota^{2} \cdot \tau$ of the second order terms in $z$ gives

$$
-\sqrt{-1} \iota^{2} \cdot \tau(z, x)=\operatorname{Im}(h(z))+f(z, z)+\rho^{\prime}(z, x)
$$

where $h$ is an analytic polynomial in $z$ with coefficients in $W^{c}, f$ is a $W$-hermitian form on $V$, and $\rho^{\prime}(z, x)=O\left(|z|^{3}+|z||x|+|x|^{2}\right)$. Then the analytic transformation $V \times W^{c} \ni(z, \xi) \rightarrow(z, \xi+h(z)) \in X$ maps $\tau(\Omega)$ onto

$$
M^{\prime}=\left\{(z, x+\sqrt{-1}(f(z, z)+\rho(z, x))) \in V \times W^{c} \mid(z, z) \in \Omega\right\}
$$

where $\rho(z, x)=\rho^{\prime}(z, x-\operatorname{Re}(h(z)))$. Note that $\rho(z, x)$ also vanishes to order $O\left(|z|^{3}+|z||x|+|x|^{2}\right)$ at 0 . Thus the proof is complete if one proves the identity $f=f_{z}$. However this is almost obvious from Lemma 2.4.6 and the convergence to $M_{f}$ of $M_{a}$ defined by (2.5.1). For, note that $V \times W$ is also regarded as the tangent space $T_{0}\left(M_{a}\right)$ of $M_{a}$. Thus we can regard the Levi-Tanaka form $f_{a}$ of $M_{a}$ at 0 as a $W$-hermitian form on $V$. Note that by the analytic correspondence $(z, \xi) \leftrightarrow\left(a z, a^{2} \xi\right)$, $a \neq 0$ a neighbourhood of 0 in $M^{\prime}$ is mapped onto a neighbourhood of 0 in $M_{a}$. Since this correspondence induces the map $T_{0}\left(M^{\prime}\right)=V \times W \ni(z, x)$ 
$\rightarrow\left(a z, a^{2} x\right) \in V \times W=T_{0}\left(M_{a}\right)$, the $W$-hermitian form $f_{a}$ does not depend on $a$; therefore $\lim _{a \rightarrow 0} f_{a}=f_{1}$, where $f_{1}$ is no other than the Levi-Tanaka form $f_{z}$ of $M$ at $z$ since we can assume $M=M^{\prime}$. On the other hand $M_{f}=\lim _{a \rightarrow 0} M_{a}$ as shown in $\S 2.5$. Since the Levi-Tanaka form of $M_{f}$ at 0 equals $f$ by Lemma 2.4.6, we conclude

$$
\begin{aligned}
f & =\lim _{a \rightarrow 0}\left(\text { the Levi-Tanaka form of } M_{a} \text { at } 0\right) \\
& =\lim _{a \rightarrow 0} f_{a}=f_{1}=f_{z},
\end{aligned}
$$

which is to be proved.

Finally we shall briefly describe a few algebraic operations between standard real submanifolds. These are very useful in producing examples to which Theorem 2.5.1 is applied.

Let $V, W$ be vector spaces over $\mathbf{C}, \mathbf{R}$ respectively, and let $f$ be a $W$-hermitian form on $V$. A linear map $\pi$ of $W$ into another real vector space $W^{\prime}$ induces naturally a linear map of $W^{c}$ into $W^{\prime c}$, which we shall denote also by $\pi$. The map $\pi \cdot f: V \times V \rightarrow W^{\prime c}$ is then a $W^{\prime}$-hermitian form on $V^{\prime}$ if $\pi$ is onto. (Recall the condition (3) of Definition 2.3.1.)

Lemma 1. Let $f, \pi$ be as above, and assume that $\pi$ is onto. If $f$ satisfies the condition $\nu_{q}$, then $\pi \cdot f$ satisfies also the condition $\nu_{q}$.

Now let $f^{\prime}$ be a $W^{\prime}$-hermitian form on a complex vector space $V^{\prime}$. Then there is a unique $W \oplus W^{\prime}$-hermitian form $g$ on $V \oplus V^{\prime}$ such that

$$
\begin{array}{ll}
g(x, y)=f(x, y) & \text { if } x, y \in V \\
g(x, y)=f^{\prime}(x, y) & \text { if } x, y \in V^{\prime} \\
g(x, y)=0 & \text { if } x \in V, y \in V^{\prime} .
\end{array}
$$

We call $g$ the direct sum of $f$ and $f^{\prime}$, and write $g=f \oplus f^{\prime}$. We set also

$$
\begin{gathered}
f \otimes f^{\prime}\left(x \otimes x^{\prime}, y \otimes y^{\prime}\right)=f(x, y) \otimes f^{\prime}\left(x^{\prime}, y^{\prime}\right) \\
x, y \in V ; \quad x^{\prime}, y^{\prime} \in V^{\prime}
\end{gathered}
$$


to define the $W \bigotimes_{\mathbf{R}} W^{\prime}$-hermitian form $f \bigotimes_{\mathbf{C}} f^{\prime}$ on $V \otimes V^{\prime}$, which we shall call the tensor product of $f$ and $f^{\prime}$.

Lemma 2. The notation being as above, $f \oplus f^{\prime}$ satisfies the condition $\nu_{q}$ if and only if both $f$ and $f^{\prime}$ satisfy the condition $\nu_{q} ; f \otimes f^{\prime}$ satisfies the condition $\nu_{q}$ if $f$ satisfies the condition $\nu_{q}$.

Since the characterization of the condition $\nu_{q}$ for $f$ is obvious in case $\operatorname{dim}_{\mathbf{R}} W=1$, Lemma 1 and Lemma 2 combined with Lemma 2.4.6 give numerous examples of standard real submanifolds satisfying the condition $\nu_{q}$.

\section{Appendix 2 \\ Spencer Sequences for Some Elliptic Systems of First Order Differential Equations with Constant Coefficients}

0. Definition of Spencer sequence. We first recall the construction of the Spencer sequences (see Spencer [15]). For a vector bundle $E$ over a manifold $M$ we denote by $J_{k}(E)$ the bundle of $k$ jets of $E$. A first order differential operator $D$ from $E$ into another vector bundle $F$ induces naturally a $\left(C^{\infty}(M)\right.$-linear) map from $J_{k+1}(E)$ to $J_{k}(E)$ for each $k \geqq 0$; we shall denote the kernel of this by $R_{k}$. Restricting the projection $J_{k+1}(E) \rightarrow J_{k}(E)$ to $R_{k+1}$, we obtain natural maps $R_{k+1} \rightarrow R_{k}, k \geqq 1$ and $R_{1} \rightarrow E$. If all of these maps are surjective, then we say that the differential equation $D u=0$ is formally integrable. We set $g_{1}=T^{*} \otimes E \cap$ $R_{1}$ in $J_{1}(E)$ where we have written $T^{*}$ for the complexified cotangent bundle of $M$. Now let $C^{0}=E$ and set $C^{i}=\Lambda^{i} T^{*} \otimes E / \delta\left(\Lambda^{i-1} T^{*} \otimes g_{1}\right)$ for $i \geqq 1$, where $\delta$ denotes the antisymmetrization.

Theorem A. Suppose that $D u=0$ is formally integrable and that $C^{i}, i>0$ are all vector bundles. Then the following statements are equivalent:

(i) $g_{1}$ is involutive. 
(ii) There exists a unique differential complex $C^{\cdot}$

$$
C^{0} \stackrel{D^{0}}{\longrightarrow} C^{1} \stackrel{D^{1}}{\longrightarrow} C^{2} \stackrel{D^{2}}{\longrightarrow} \cdots
$$

satisfying the following conditions:

a) $D u=0$ if and only if $D_{u}^{0}=0$ for every local section $u$ of $E$.

b) $C \cdot$ is formally exact.

c) The principal symbol of $D^{i}, \sigma\left(D^{i}\right): T^{*} \otimes C^{i} \rightarrow C^{i+1}$ is induced by the antisymmetrization $T^{*} \otimes \Lambda^{i} T^{*} \otimes E \rightarrow \Lambda^{i+1} T^{*} \otimes E$.

For the proof see Goldschmidt [2] or Quillen [13]. The complex $C^{\text {. }}$ is usually called the Spencer sequence for the equation $D u=0$, and has the following important property:

Theorem B (Quillen [13]). In addition to the hypothesis of Theorem $A$, assume that $g_{1}$ is involutive. Then, for every complex cotangent vector $\xi \in T_{z}^{*}, z \in M$, the symbol $\sigma_{\xi}(D)$ at $\xi$ is injective if and only if the symbol sequence

$$
0 \longrightarrow C_{z}^{0} \stackrel{\sigma_{\xi}\left(D^{0}\right)}{\longrightarrow} C_{z}^{1} \stackrel{\sigma_{\xi}\left(D^{1}\right)}{\longrightarrow} C_{z}^{2} \longrightarrow \cdots
$$

is exact. In particular $D$ is elliptic if and only if $C^{\cdot}$ is an elliptic complex.

1. Main Theorem. Here we only state our main theorem. We shall first list up the assumptions for differential operators $D: E \rightarrow F$ to which our theorem is applied:

(i) $E, F$ are trivial bundles over $\mathbf{C}^{N}$, and $D$ is a homogeneous differential operator of order 1 with constant coefficients.

(ii) $f D u=D f u$ if $f$ is analytic in $\mathbf{C}^{N}$.

(iii) $D$ is elliptic.

(iv) For every complex cotangent vector, the symbol $\sigma_{\xi}(D)$ is either injective, or 0.

(v) $g_{1}$, defined above is involutive.

Note that the homogeneity of $D$ implies that $D u=0$ is formally integrable, so that the Spencer sequence $C \cdot$ for $D u=0$ is well defined. 
Theorem 1. Let $D$ satisfy the assumptions (i) $\sim(\mathrm{v})$, and assume that $K$ is a polynomially convex compact subset of $\mathbf{C}^{N}$. Then the sequence

$$
\Gamma\left(K, \underline{G}^{0}\right) \stackrel{D^{0}}{\longrightarrow} \Gamma\left(K, \underline{C}^{1}\right) \stackrel{D^{1}}{\longrightarrow} \Gamma\left(K, \underline{C}^{2}\right) \stackrel{D^{2}}{\longrightarrow}
$$

is exact.

To prove this theorem we must study some type of short complexes $C^{\infty}\left(\mathbf{R}^{n}, \mathbf{C}^{r}\right) \stackrel{Q}{\longrightarrow} C^{\infty}\left(\mathbf{R}^{n}, \mathbf{C}^{q}\right) \stackrel{P}{\longrightarrow} C^{\infty}\left(\mathbf{R}^{n}, \mathbf{C}^{p}\right)$ where $P, Q$ are homogeneous, first order differential operators with constant coefficients.

2. Study of short complexes. We denote by $|x|,|z|$ the euclidean norms $\left(\left|x_{1}\right|^{2}+\cdots\left|x_{n}\right|^{2}\right)^{1 / 2},\left(\left|z_{1}\right|^{2}+\cdots+\left|z_{n}\right|^{2}\right)^{\frac{1}{2}}$ of $\mathbb{R}^{n}, \mathbb{C}^{n}$ respectively. Choose positive integers $p, q, r$, and let $P_{k}, k=1,2, \ldots, n$ be $(p, q)$ matrices with complex entries and $Q_{k}, k=1,2, \ldots, n$ be $(q, r)$ matrices with complex entries. Introduce differential operators on $\mathbf{R}^{n} P\left(\frac{\partial}{\partial x}\right)$, $Q\left(\frac{\partial}{\partial x}\right)$ setting

$$
\begin{aligned}
& P\left(\frac{\partial}{\partial x}\right)=\sum_{k} P_{k} \frac{\partial}{\partial x_{k}} \\
& Q\left(\frac{\partial}{\partial x}\right)=\sum_{k} Q_{k} \frac{\partial}{\partial x_{k}} .
\end{aligned}
$$

For these we assume the following assumptions:

(A1) $P\left(\frac{\partial}{\partial x}\right) Q\left(\frac{\partial}{\partial x}\right)=0$.

(A2) If a q-tuple of polynomials $u$ satisfies the equation $P\left(\frac{\partial}{\partial x}\right) u$ $=0$, then there is $u \in C^{\infty}\left(\mathbf{R}^{n}\right)^{r}$ such that $Q\left(\frac{\partial}{\partial x}\right) v=u$.

(A3) $\quad \mathbf{C}^{r} \stackrel{Q(\xi)}{\longrightarrow} \mathbf{C}^{q} \stackrel{P(\xi)}{\longrightarrow} \mathbf{C}^{p}$ is either exact, or 0 for any $\xi \in \mathbf{C}^{n}$.

Since $Q\left(\frac{\partial}{\partial x}\right)$ is homogeneous, the assumption (A2) can be replaced by the following equivalent but seemingly stronger assumption:

(A2)' For every $q$-tuple of homogeneous polynomials $u$ such that 
$P\left(\frac{\partial}{\partial x}\right) u=0$, one can find a r-tuple of homogeneous polynomials $v$ such that $u=Q\left(\frac{\partial}{\partial x}\right) v$.

Now the main objective in this section is to prove the following

Theorem 2. Let the assumptions (A1), (A2), (A3) be fulfilled. Then there is a constant $C>0$ such that

$$
\begin{aligned}
& \int|u|^{2} e^{-\frac{|x|^{2}}{2}} d x \leqq C\left(\int\left|Q^{*}\left(\frac{\partial}{\partial x}-x\right) u\right|^{2} e^{-\frac{|x|^{2}}{2}} d x\right. \\
& \left.+\int\left|P\left(\frac{\partial}{\partial x}\right) u\right|^{2} e^{-\frac{|x|^{2}}{2}} d x\right) \\
& u \in C_{0}^{\infty}\left(\mathbf{R}^{n}\right)^{q} .
\end{aligned}
$$

Here we have set $Q^{*}(\xi)=\sum_{k=1}^{n} Q_{k}^{*} \xi_{k}$ with conjugate transposed matrices $Q_{k}^{*}$ of $Q_{k}, k=1,2, \ldots, n$.

To prove this theorem we first introduce several Hilbert spaces: Introduce the Gaussian measure $d G=(2 \pi i)^{-n} e^{-|z|^{2} / 2} d z_{1} d \bar{z}_{1} d z_{2} d \bar{z}_{2} \ldots d z_{n} d \bar{z}_{n}$ on $\mathbf{C}^{n}:\left(z_{1}, \cdots, z_{n}\right)$ and put

$$
H_{z}=\left\{\left.f \in A\left(\mathbf{C}^{n}\right)\left|\int\right| f\right|^{2} d G<+\infty\right\}
$$

where we have written $A\left(\mathbf{C}^{n}\right)$ for the set of analytic entire functions on $\mathbf{C}^{n}$. The space $H_{z}$ is also a Hilbert space with the norm $\|f\|=$ $\left(\int|f|^{2} d G\right)^{\frac{1}{2}}$. Obviously all the (analytic) polynomials belong to $H_{z}$. Integrating by parts we obtain

$$
\left(\frac{\partial u}{\partial z}, v\right)=\left(u, z_{i} v\right)
$$

for analytic polynomials $u, v$ in $z$, where $($,$) denotes the polar form of$ \|\|$^{2}$, of course. From (a.2) it follows the following orthogonality relation:

$$
\left(z^{\alpha}, z^{\beta}\right)=\alpha ! \delta_{\alpha \beta}
$$


for multi-indices $\alpha, \beta$. The system $\left(z^{\alpha}\right)_{\alpha}$, where $\alpha$ ranges over all multiindices, that is, all $n$-tuple of non-negative integers, is a complete orthogonal system. In fact the Taylor series for $u \in H_{z}$ is identical with the Fourier series of $u$ by this system in $H_{z}$. For later convenience we denote by $H_{z, k}$ the subspace of $H_{z}$ spanned by monomials $z^{\alpha},|\alpha|=k$.

Let $H_{x}$ be the Hilbert space with norm \|\| which consists of all locally square-integrable functions $f$ on $\mathbf{R}^{n}$ such that $\|f\|^{2}=(2 \pi)^{-n / 2} \int|f|^{2}$ $e^{-|x|^{2} / 2} d x$. To introduce a suitable orthogonal system for $H_{x}$ we define the Hermite polynomials $H_{n}(t)$ on the real line $(-\infty<t<+\infty)$ by

$$
H_{n}(t)=(-1)^{n} e^{\frac{t^{2}}{2}}\left(\frac{d}{d t}\right)^{n}\left(e^{-\frac{t^{2}}{2}}\right)
$$

The following formulas are well known:

$$
\begin{gathered}
\int H_{m}(t) H_{n}(t) e^{-\frac{t^{2}}{2}} d t=m ! \delta_{m n} \\
\frac{d}{d t} H_{n}(t)=n H_{n-1}(t) .
\end{gathered}
$$

The completeness of the system $\left(H_{n}(t)\right)_{n \geqq 0}$ is also well known, so, if one sets for multi-index $\alpha=\left(\alpha_{1}, \ldots, \alpha_{n}\right)$

$$
H_{\alpha}(x)=H_{\alpha_{1}}\left(x_{1}\right) H_{\alpha_{2}}\left(x_{2}\right) \ldots H_{\alpha_{n}}\left(x_{n}\right),
$$

then the family $\left(H_{\alpha}(x)\right)_{\alpha}$ is a complete orthogonal system for $H_{x}$

Let now $u(x)=\sum_{\alpha} b_{\alpha} H_{\alpha}(x)$ and $v(z)=\sum_{\alpha} c_{\alpha} z^{\alpha}$ be the Fourier series of $u \in H_{x}$ and of $v \in H_{z}$ respectively. We set

$$
\begin{aligned}
& u_{k}=\sum_{|\alpha|=k} b_{\alpha} H^{\alpha}(x) \\
& u_{k}=\sum_{|\alpha|=k} c_{\alpha} z^{\alpha} .
\end{aligned}
$$

Since the maps $u \mapsto u_{k}, v \mapsto v_{k}$ are orthogonal projections, we have

$$
\begin{array}{ll}
\left(u_{k}, u^{\prime}\right)=\left(u_{k}, u_{k}^{\prime}\right)=\left(u, u_{k}^{\prime}\right) & u, u^{\prime} \in H_{x} \\
\left(v_{k}, v^{\prime}\right)=\left(v_{k}, v_{k}^{\prime}\right)=\left(v, v_{k}^{\prime}\right) & v, v^{\prime} \in H_{z} .
\end{array}
$$


As we have done in $\S 1.4$, the differential operators $P\left(\frac{\partial}{\partial x}\right), Q\left(\frac{\partial}{\partial x}\right)$, $P\left(\frac{\partial}{\partial z}\right), Q\left(\frac{\partial}{\partial z}\right)$ define linear, closed, densely defined operators. $S_{x}: H_{x}^{q} \rightarrow$ $H_{x}^{p}, T_{x}: H_{x}^{r} \rightarrow H_{x}^{q}, S_{z}: H_{z}^{q} \rightarrow H_{z}^{p}, T_{z}: H_{z}^{r} \rightarrow H_{z}^{q}$ if one puts $D\left(S_{x}\right)=$ the set of $u \in H_{x}^{q}$ such that $P\left(\frac{\partial}{\partial x}\right) u$, defined in the distribution sense, lies in $H_{x}^{p}$, and similarly for $D\left(T_{x}\right), D\left(S_{z}\right), D\left(T_{z}\right)$. By the homogeneity of $P\left(\frac{\partial}{\partial x}\right)$, $\ldots$, we obtain immediately

$$
\begin{array}{ll}
T_{x}\left(u_{k}\right)=\left(T_{x} u\right)_{k-1} & u \in D\left(T_{x}\right) \\
S_{x}\left(u_{k}\right)=\left(S_{x} u\right)_{k-1} & u \in D\left(S_{x}\right) \\
T_{z}\left(v_{k}\right)=\left(T_{z} v\right)_{k-1} & v \in D\left(T_{z}\right) \\
S_{z}\left(v_{k}\right)=\left(S_{z} v\right)_{k-1} & v \in D\left(S_{z}\right) .
\end{array}
$$

We obtain also from (a.6) and (a.7)

$$
\begin{array}{ll}
T_{x}^{*}\left(u_{k}\right)=\left(T_{x}^{*} u\right)_{k+1} & u \in D\left(T_{x}^{*}\right) \\
T_{z}^{*}\left(u_{k}\right)=\left(T_{z}^{*} u\right)_{k+1} & u \in D\left(T_{z}^{*}\right) .
\end{array}
$$

Let $u_{(k)}=\sum_{l \leqq k} u_{l}$ and $v_{(k)}=\sum_{l \leqq k} v_{l}$ for $u \in H_{x}$ and $v \in H_{z}$. Then the formulas (a.7)-(a.8) together with the fact that $\left\|u-u_{(k)}\right\| \rightarrow 0$ $\left\|u-v_{(k)}\right\| \rightarrow 0 \quad(k \rightarrow \infty)$, imply

Lemma 2. The set of q-tuples of polynomials in $x$ (resp. in $z$ ) is dense in $D\left(T_{x}^{*}\right) \cap D\left(S_{x}\right)$ (resp. in $D\left(T_{z}^{*}\right) \cap D\left(S_{z}\right)$ ) for the graph norm $\|u\|+\left\|T_{x}^{*} u\right\|+\left\|S_{x} u\right\|$ (resp. for $\left.\|v\|+\left\|T_{z}^{*} v\right\|+\left\|S_{z} u\right\|\right)$.

Now we introduce a one-to-one correspondence $H_{x} \ni u \rightarrow \hat{u} \in H_{z}$ which assigns $z^{\alpha}$ to $H_{\alpha}(x)$ for every multi-index $\alpha$. This correspondence is unitary by (a.3) and (a.4), i.e. preserving the norms, and is called the Gauss-Laplace transformation. It follows from (a.5) that the GaussLaplace transformation preserves the differentiation. Thus, in particular we obtain

Lemma 3. An element $u \in H_{x}$ belongs to $D\left(T_{x}\right)$ (resp. $D\left(S_{x}\right)$ ) if 
and only if $\hat{u}$ belongs to $D\left(T_{z}\right)\left(\operatorname{resp.} D\left(S_{z}\right)\right)$. For $u \in D\left(T_{x}\right)\left(\operatorname{resp} . D\left(S_{x}\right)\right)$ it holds $T_{x} u=T_{z} \hat{u}$ (resp. $\left.S_{x} u=S_{z} \hat{u}\right)$.

With the aid of this lemma we can reduce the estimate (a.1) to the following equivalent one:

$$
\|v\| \leqq C\left(\left\|T_{z}^{*}\right\|+\left\|S_{z} v\right\|\right) \quad v \in D\left(T_{z}^{*}\right) \cap D\left(S_{z}\right)
$$

In fact (a.1), (a.9) are equivalent to the identities $N\left(S_{x}\right)=R\left(T_{x}\right)$, $N\left(S_{z}\right)=R\left(T_{z}\right)$ respectively. (See Lemma 1.4.2.) Moreover these identities are the same by Lemma 3. The estimate (a.9) is rather advantageous than (a.1), the reason for which we shall now explain below: First note that, if $u \in D\left(T_{z}^{*}\right)$, then

$$
T_{z}^{*} u(z)=Q^{*}(z) u(z),
$$

hence

$$
\left\|T_{z}^{*} u\right\|^{2}=\int\left|Q^{*}(z) u(z)\right|^{2} d G
$$

by the formula (a.2). Using (a.2) we can also transform $\left\|S_{z} u\right\|^{2}$ as follows :

$$
\begin{aligned}
\left\|S_{z} u\right\|^{2} & =\left(\sum_{k} P_{k} \frac{\partial u}{\partial z_{k}}, \sum_{l} P_{l} \frac{\partial u}{\partial z_{l}}\right) \\
& =\sum_{k, l}\left(P_{k} u, P_{l} z_{k} \frac{\partial u}{\partial z_{l}}\right) \\
& =\sum_{k, l}\left(P_{k} u, P_{l} \frac{\partial\left(z_{k} u\right)}{\partial z_{l}}\right)-\sum_{k}\left(P_{k} u, P_{k} u\right) \\
& =\sum_{k, l}\left(P_{k} z_{l} u, P_{l} z_{k} u\right)-\sum_{k}\left(P_{k} u, P_{k} u, P_{k} u\right) \\
& =\int|P(\bar{z}) u(z)|^{2} d G-\sum_{k} \int\left|P_{k} u\right|^{2} d G .
\end{aligned}
$$

Therefore we obtain

$$
\left\|T_{z} u\right\|^{2}+\left\|S_{z} u\right\|^{2}=\int E(\bar{z}, u(z)) d G-\sum_{k}\left|P_{k} u\right|^{2} d G
$$


where we have set for $\xi \in \mathbf{C}^{n}$ and for $u \in \mathbf{C}^{q}$

$$
E(\xi, u)=\left|Q^{*}(\xi) u\right|^{2}+|P(\xi) u|^{2}=\left|Q(\xi)^{*} u\right|^{2}+|P(\xi) u|^{2} .
$$

Note that no differentiation occurs in the right hand side of (a.10). This is the reason why we prefer (a.9) to (a.1).

To prove Theorem 2 we still need some estimates concerning several norms for analytic polynomials which are used in Quillen [14]: Following Quillen we set for an analytic polynomial $u$

$$
\begin{gathered}
\|u\|_{q}^{2}=\sum_{|\gamma|=q}(\gamma !)^{-1} \int\left|\left(\frac{\partial}{\partial z}\right)^{\gamma} u\right|^{2} d G \\
\|u\|_{q}^{2}=(q !)^{-1} \int|z|^{2 q}|u|^{2} d G .
\end{gathered}
$$

Let $\left(\begin{array}{l}k \\ q\end{array}\right)$ be the binomial coefficients.

Lemma 4 (Quillen). $\|u\|_{q}^{2}=\left(\begin{array}{c}k \\ q\end{array}\right)\|u\|_{0}$ for $u \in H_{z, k}$.

Lemma 5 (Quillen). There is a constant $C$ depending only on $q$ such that

$$
\|u\|_{q}^{2} \leqq\|u\|_{q^{*}}^{2} \leqq C \sum_{j \leqq q}\|u\|_{j}^{2}
$$

Proof of Theorem 2. As remarked above, we may only prove the estimate

$$
\|u\|^{2} \leqq C\left(\left\|T_{z}^{*} u\right\|^{2}+\left\|S_{z} u\right\|^{2}\right)
$$

for analytic polynomials $u$. (i) We first prove this when the sequence

$$
\mathbf{C}^{r} \stackrel{Q(\xi)}{\longrightarrow} \mathbf{C}^{q} \stackrel{P(\xi)}{\longrightarrow} \mathbf{C}^{p}
$$

is exact for every non zero $\xi \in \mathbf{C}^{n}$. In this case there is a constant $\varepsilon>0$ such that

$$
E(\xi, u)=\left|Q(\xi)^{*} u\right|^{2}+|P(\xi) u|^{2} \geqq \varepsilon|u|^{2} .
$$

Using Lemma 5 we obtain from (a.10) 


$$
\begin{aligned}
\left\|T_{z}^{*} u\right\|^{2}+\left\|S_{z} u\right\|^{2} & \geqq \varepsilon\|u\|_{1^{*}}^{2}-C^{\prime}\|u\|_{0}^{2} \\
& \geqq \varepsilon\|u\|_{1}^{2}-C^{\prime}\|u\|_{0}^{2}
\end{aligned}
$$

where we have chosen constant $C^{\prime}>0$ so that $\sum_{k}\left|P_{k} u\right|^{2} \leqq C^{\prime}|u|^{2}$. Thus, by Lemma 2.2 .4

$$
\left\|T_{z}^{*}\right\|^{2}+\left\|S_{z} u\right\|^{2} \geqq\left(\varepsilon k-C^{\prime}\right)\|u\|^{2} \quad u \in\left(H_{z, k}\right)^{q} .
$$

On the other hand the sequence

$$
\left(H_{z, k+1}\right)^{r} \stackrel{T_{z}}{\longrightarrow}\left(H_{z, k}\right)^{q} \stackrel{S_{z}}{\longrightarrow}\left(H_{z, k-1}\right)^{p}
$$

is exact by the assumption (A2). Hence there is a positive constant such $C_{k}$ that

$$
\left\|T_{z}^{*} u\right\|^{2}+\left\|S_{z} u\right\|^{2} \geqq C_{k}\|u\|^{2} \quad u \in\left(H_{z, k}\right)^{q} .
$$

Find an integer $k_{0}$ such that $\varepsilon k_{0}-C^{\prime}>0$ and set $C=\min \left(C_{0}, C_{1}, \ldots\right.$, $C_{k_{0}-1}, \varepsilon k_{0}-C^{\prime}$ ). Since $\|u\|^{2}=\sum_{k}\left\|u_{k}\right\|^{2}$ by (a.6), the combination of (a.7), (a.11) and (a.12) implies

$$
\left\|T_{z}^{*} u\right\|^{2}+\left\|S_{z} n\right\|^{2} \geqq C\|u\|^{2} \quad u \in D\left(T_{z}^{*}\right) \cap D\left(S_{z}\right) .
$$

(ii) Now we shall prove $\left(^{*}\right)$ without the assumption imposed at the beginning of (i). By a suitable unitary transformation of the coordinates $\left(z_{1}, \ldots, z_{n}\right)$ we may assume the subspace $\left\{\xi \in \mathbf{C}_{n} \mid P(\xi)=Q(\xi)=0\right\}$ of $\mathbf{C}^{n}$ is characterized by $\xi_{1}=\xi_{2} \ldots=\xi_{\rho}=0$. Given a vector $\xi=\left(\xi_{1}, \ldots, \xi_{n}\right) \in \mathbf{C}^{n}$ we write $\xi^{\prime}, \xi^{\prime \prime}$ for $\left(\xi_{1}, \ldots, \xi_{\rho}\right),\left(\xi_{\rho+1}, \ldots, \xi_{n}\right)$. Then the assumption (A3) implies that there is a constant $\varepsilon>0$ such that

$$
E(\xi, u)=E\left(\xi^{\prime}, u\right) \geqq\left|\xi^{\prime}\right|^{2}|u|^{2} \quad \xi \in \mathbf{C}, u \in \mathbf{C}^{q} .
$$

Thus, as just proved above, there is a constant $C>0$ such that

$$
\begin{array}{r}
\frac{1}{(2 \pi i)^{\rho}} \int\left(\left|T_{z}^{*} u\right|^{2}+\left|S_{z} u\right|^{2}\right) e^{-\left|z^{\prime}\right|^{2}} d z_{1} d \bar{z}_{1} \cdots d z_{\rho} d \bar{z}_{\rho} \\
\geqq \frac{C}{(2 \pi i)^{\rho}} \int|u|^{2} e^{-\left|z^{\prime}\right|^{2}} d z_{1} d \bar{z} \cdots d z_{\rho} d \bar{z}_{\rho}
\end{array}
$$

for analytic polynomials $u$. Here both hand sides still depend on $z^{\prime \prime}$. 
We integrate this inequality over $\mathbf{C}^{n-\rho}: \boldsymbol{z}^{\prime \prime}$ after multiplying both hand sides by

$$
\frac{1}{(2 \pi i)^{n-\rho}} e^{-\left|z^{\prime \prime}\right|^{2}} d z_{\rho+1} d \bar{z}_{\rho+1} \cdots d z_{n} d \bar{z}_{n}
$$

then

$$
\left\|T_{z}^{*} u\right\|^{2}+\left\|S_{z} u\right\|^{2} \geqq C\|u\|
$$

Thus the theorem is completely proved.

3. Proof of Theorem 1. We shall prove Theorem 1 applying Theorem 2 to the Spencer sequence $C \cdot$ for $D u=0$. Let the hypothesis of Theorem 1 be fulfilled. By Theorem $\mathrm{A}$, the Spencer sequence $C^{*}$ is then formally exact. Hence the assumption (A2) is fulfilled for each $i>0$ if one sets $P\left(\frac{\partial}{\partial x}\right)=D^{i}, Q\left(\frac{\partial}{\partial x}\right)=D^{i-1}$. (Note that $C^{i}, i \geqq 0$ are all regarded as trivial bundles and $D^{i}$ are differential operators with constant coefficients. This follows from the translation invariance of $D u=0$ and from the naturality of the Spencer sequences.) Recall that $\sigma_{\xi}(D)$ is either injective, or 0 for every complex cotangent vector $\xi$. Thus the assumption (A3) is also fulfilled with $P\left(\frac{\partial}{\partial x}\right), Q\left(\frac{\partial}{\partial x}\right)$ replaced by $D^{i}, D^{i-1}(i>0)$. This follows from Theorem B. Thus we can apply Theorem 2 to $P\left(\frac{\partial}{\partial x}\right)=D^{i}, Q\left(\frac{\partial}{\partial x}\right)=D^{i-1}$ and we obtain

$$
\|u\|_{i}^{2} \leqq C_{i}\left(\left\|\left(D^{i-1}\right)^{*} u\right\|_{i-1}+\left\|D^{i} u\right\|_{i+1}\right) \quad u \in C_{0}^{\infty}\left(\mathbf{C}^{n}, C^{i}\right)
$$

where we have chosen translation invariant Hermitian inner products $<,>_{i}$ for trivial bundles $C^{i}$, and we have set

$$
\begin{aligned}
& |u|_{i}^{2}=\langle u, u\rangle \\
& \|u\|_{i}^{2}=\int|u|_{i}^{2} d G . \quad u \in C_{0}^{\infty}\left(\mathbf{C}^{n}, C^{i}\right) .
\end{aligned}
$$

Hence the assumption $\left(\mathscr{B}_{1}\right)$ in $\S 1.4$ is fulfilled for the complex $C^{\cdot}=(\cdots \rightarrow$ $\left.0 \rightarrow C^{0} \rightarrow C^{1} \rightarrow \cdots\right)$. Thus, to finish the proof of Theorem 1, we may only 
find a good subring of the ring of $C^{*}$-analytic functions which is well filtered in the sense of Definition 1.4.4. Note the assumption (ii) in $\mathbf{1}$ implies that every analytic function in $\mathbf{C}^{N}$ is $C^{*}$-analytic. Let $P_{k}$ be the set of analytic polynomials of degree not larger than $k$. Let $\varphi(z)$ be a smooth function such that $\varphi(z)=|z|$ outside some compact subset of $\mathbb{C}^{N}$, and set $\chi_{\nu}(z)=e^{-\nu^{-1} \varphi(z)}$. Then one can easily check that the conditions of Definition 1.4 .4 are fulfilled by $P_{0} \subset P_{1} \subset P_{2} \subset \cdots$ and $x_{\nu} \nu=1,2, \cdots$, that is, $P=\bigcup_{k} P_{k}$ is well filtered. Thus Theorem 1.4.5 combined with Corollary 1.3.2 completes the proof.

\section{References}

[1] Ehrenpreis, L., A new proof and an extension of Hartogs theorem, Bull. Amer. Math. Soc. 67 (1961), 507-509.

[2] Goldschmidt, H., Existence theorems for analytic linear partial differential equations, Ann. of Math. 86 (1967), 246-270.

[3] Greenfield, Cauchy-Riemann equation in several variables, Ann. Scuola Norm. Sup. Pisa, 22 (1968), 175-314.

[4] Hörmander, L., $L^{2}$ estimates and existence theorems for the $\bar{\partial}$ operator, Acta Math. 113 (1965), 89-152.

[5] - An Introduction to Complex Analysis in Several Variables, Van Nostrand, 1966.

[6] - Pseudo-differential operators and nonelliptic boundary problems, Ann. of Math. 83 (1966), 129-209.

[7] - Hypoelliptic second order differential equations, Acta Math. 119 (1967), 147-171.

[8] Kohn, J. J., Boundaries of complex manifolds, Proc. Minneapolis Conference on Complex Analysis, Springer-Verlag, Berlin, 1965, 81-94.

[9] Kohn, J. J. and H. Rossi, On the extension of holomorphic functions from the boundary of a complex manifold, Ann. of Math. 81 (1965), 451-472.

[10] MacKichan, B., A generalization to overdetermined systems of the notion of diagonal operators, Acta Math. 126 (1971), 83-119.

[11] Naruki, I., Holomorphic extension problem for standard real submanifolds of second kind, Publ. RIMS Kyoto Univ. 6 (1970), 113-187.

[12] - Localization principle in differential complexes and its application, to appear in Proc. Japan Acad.

[13] Quillen, D. G., Formal properties of overdetermined systems of linear partial differential equations, Thesis, Harvard University, 1964 (unpublished).

[14] - On the representation of Hermitian forms as sums of squares, Invent. Math. 5 (1968), 237-242.

[15] Spencer, D. C., Overdetermined systems of linear partial differential equations, Bull. Amer. Math. Soc. 75 (1969), 179-239.

[16] Sweeney, W. J. The D-Neumann problem, Acta Math. 120 (1968), 223-277.

[17] —, A uniqueness theorem for the Neumann problem, Ann. of 
Math. 90 (1969), 353-360.

[18] Tanaka, N., On differential systems, Lie algebras and pseudo-groups, J. Math Kyoto Univ. 10 (1970), 1-82. 